Multiphysics Design Optimization Model for Structural Walls Incorporating

Phase Change Materials

By

Amie Stockwell

\begin{abstract}
A Thesis Presented in Partial Fulfillment of the Requirements for the Degree Master of Science
\end{abstract}

Approved May 2013 by the Graduate Supervisory Committee:

Subramaniam Rajan, Co-Chair Narayanan Neithalath, Co-Chair Barzin Mobasher

\title{
ARIZONA STATE UNIVERSITY
}

August 2013 


\begin{abstract}
Buildings consume a large portion of the world's energy, but with the integration of phase change materials (PCMs) in building elements this energy cost can be greatly reduced. The addition of PCMs into building elements, however, becomes a challenge to model and analyze how the material actually affects the energy flow and temperatures in the system. This research work presents a comprehensive computer program used to model and analyze PCM embedded wall systems.

The use of the finite element method (FEM) provides the tool to analyze the energy flow of these systems. Finite element analysis (FEA) can model the transient analysis of a typical climate cycle along with nonlinear problems, which the addition of PCM causes. The use of phase change materials is also a costly material expense. The initial expense of using PCMs can be compensated by the reduction in energy costs it can provide. Optimization is the tool used to determine the optimal point between adding PCM into a wall and the amount of energy savings that layer will provide. The integration of these two tools into a computer program allows for models to be efficiently created, analyzed and optimized.
\end{abstract}

The program was then used to understand the benefits between two different wall models, a wall with a single layer of PCM or a wall with two different PCM layers. The effect of the PCMs on the inside wall temperature along with the energy flow across the wall are computed. The numerical results show that a multi-layer PCM wall was more energy efficient and cost effective than the single PCM layer wall. A structural analysis was then performed on the optimized designs using ABAQUS v. 6.10 to ensure the structural integrity of the wall was not affected by adding PCM layer(s). 


\section{ACKNOWLEDGMENTS}

I would like to express my sincere gratitude to my advisors and committee chairs, Dr. Rajan and Dr. Neithalath for their continuous support and help throughout my studies. Their mentoring, encouragement and supervision has allowed me to learn and grow in my research work and develop invaluable skills. I would also like to extend my gratitude to Dr. Mobasher for being on my thesis committee.

I would also like to thank my family and friends for all their support and motivation throughout my years of study. I would like to acknowledge all of my classmates and colleagues, especially Lavannya, Hembade, Canio Hoffarth, Ussala Chowdhury, Arumugam Deivanayagam, Breeann Sharma, Ben Rehder and Kirk Vance for all their support and assistance. I would also like to acknowledge all of the CEE staff for their help and support.

I would like to dedicate this thesis to my parents, Dave and Julie Stockwell, and fiancé, Tyler Baisley, and to all my family and friends. 


\section{TABLE OF CONTENTS}

Page

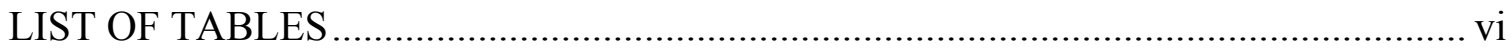

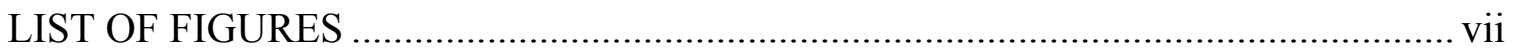

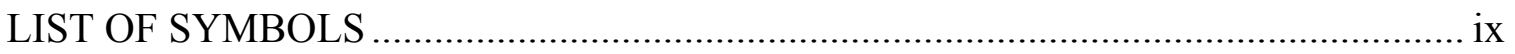

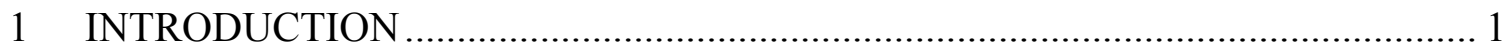

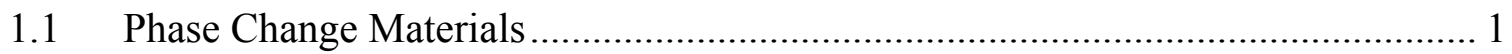

$1.2 \quad$ Finite Element Analysis used with PCMs ......................................................... 4

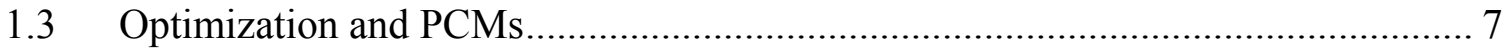

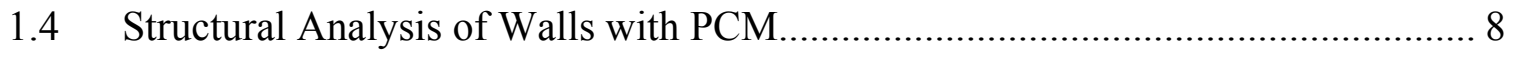

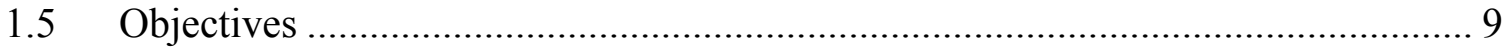

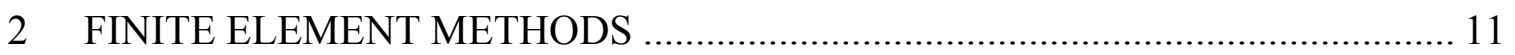

2.1 Linear Steady State Analysis ..................................................................... 13

2.1.1 Implementation in the IBVP Program........................................................ 15

2.2 Linear Transient Analysis ............................................................................. 17

2.2.1 Implementation into the IBVP Program............................................................ 19

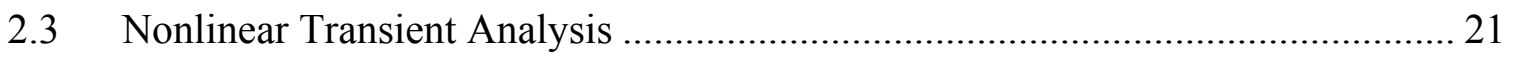

2.3.1 Temperature Dependent Conductivity ………........................................... 21

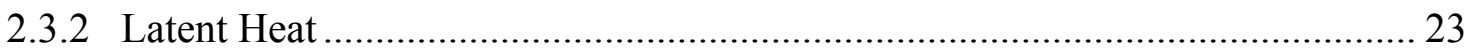

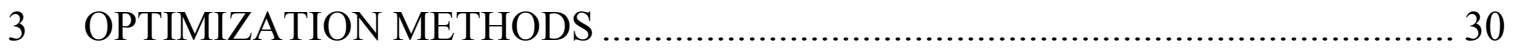

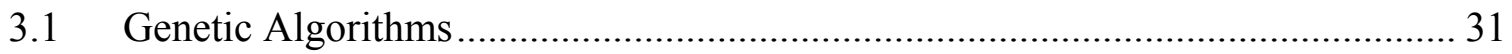

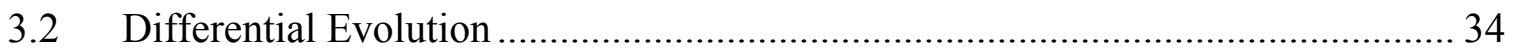

3.3 Implementation into the WallDesign Program …….............................................. 35 


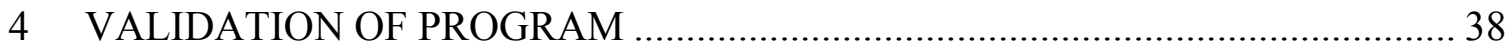

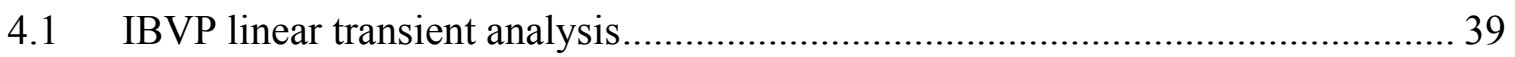

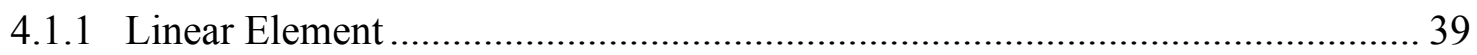

4.1.2 Quadratic Element ....................................................................................... 42

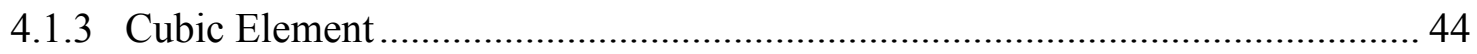

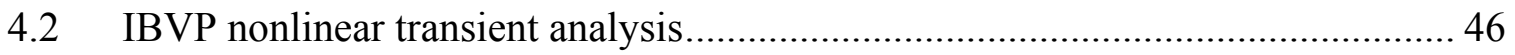

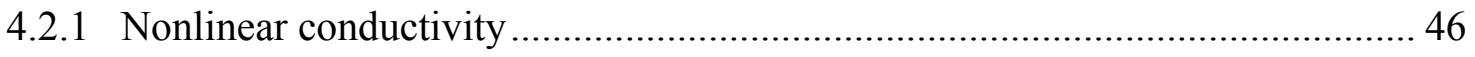

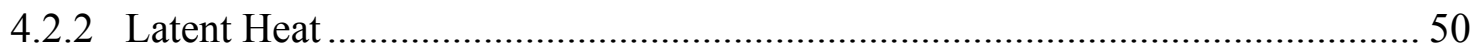

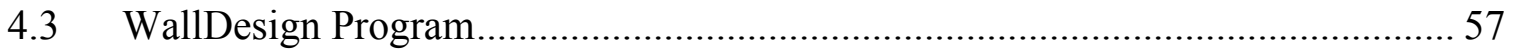

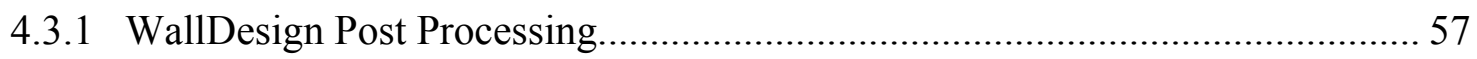

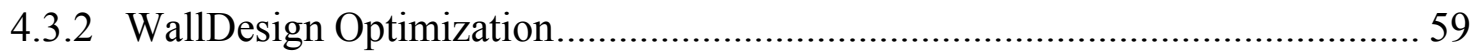

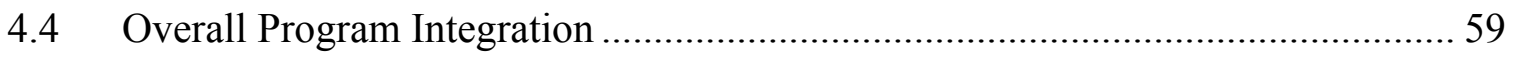

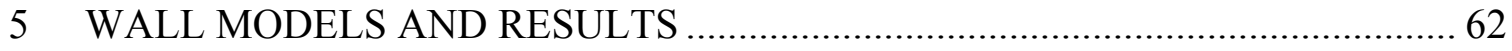

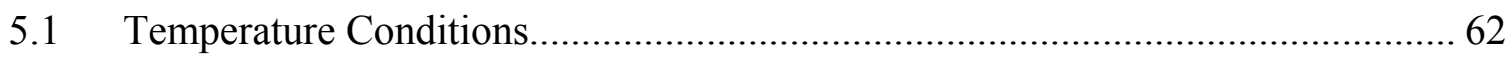

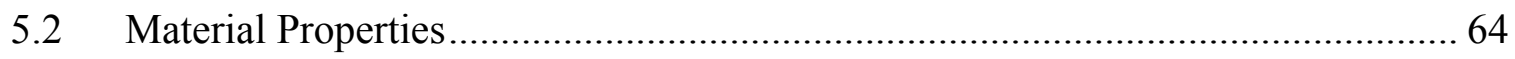

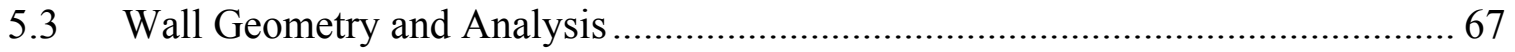

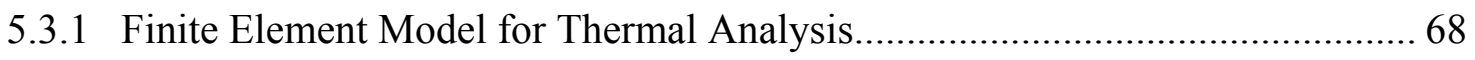

5.3.2 Finite Element Model for Structural Performance............................................ 70

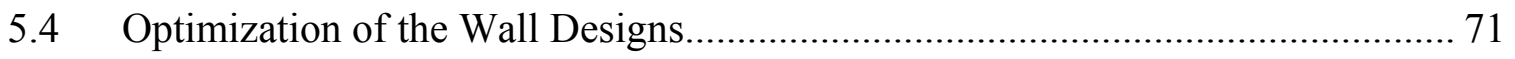

5.4.1 Stage 1 Optimization (Material Selection) …………....................................... 74

5.4.2 Stage 2 Optimization (Layer Thickness).......................................................... 76

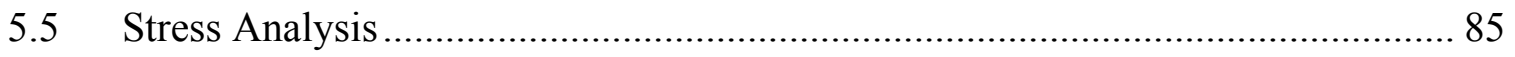

5.6 Comparison of IBVP Results to ABAQUS …………......................................... 86 


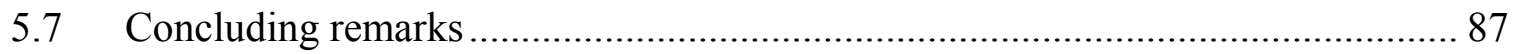

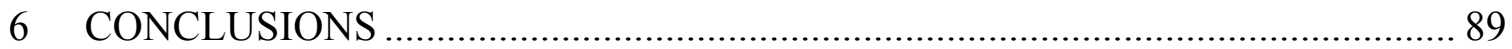

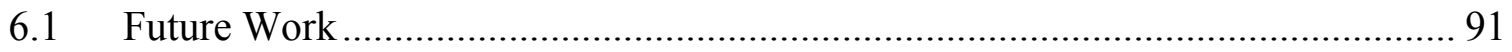

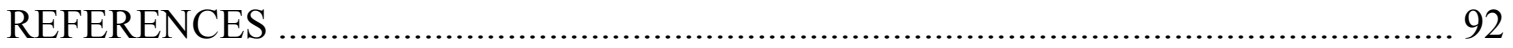

APPENDIX A WALLDESIGN PROGRAM ......................................................... 98

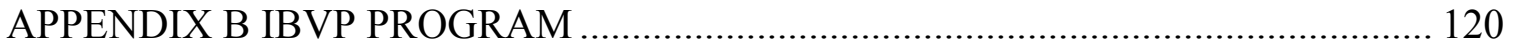




\section{LIST OF TABLES}

Table 4-1: Time Step Data for Linear Element ........................................................ 40

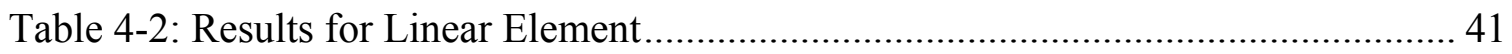

Table 4-3: Time Step Data for Quadratic Element .................................................... 42

Table 4-4: Results for Quadratic Element ............................................................. 43

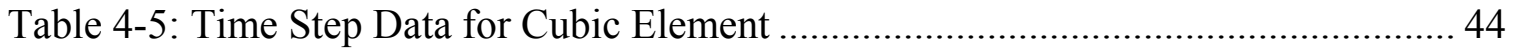

Table 4-6: Results for Cubic Element.................................................................. 45

Table 4-7: Results for six element nonlinear analysis .............................................. 48

Table 4-8: Results for two hundred element nonlinear analysis ................................ 49

Table 4-9: Results for 8 element nonlinear analysis ................................................ 52

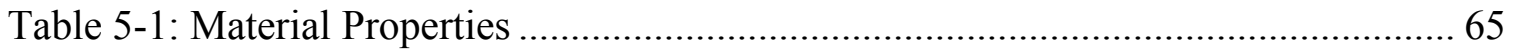

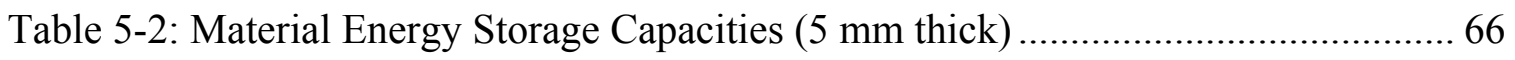

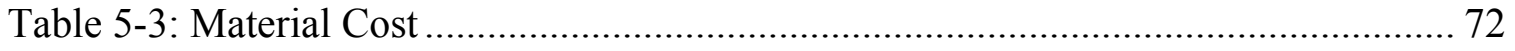

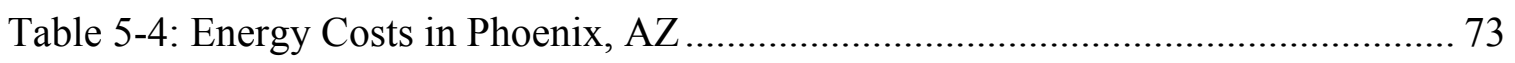

Table 5-5: Baseline Design Comparison ............................................................ 75

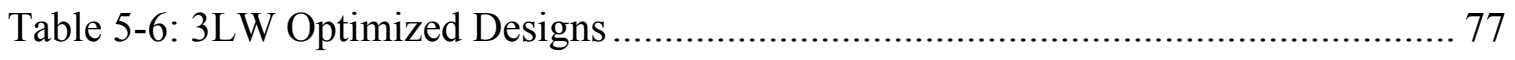

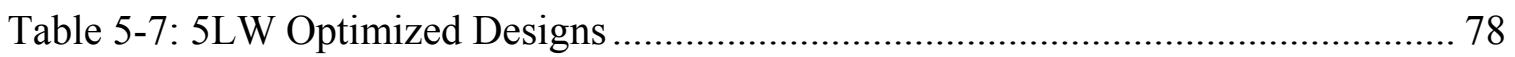

Table 5-8: Comparison of IBVP program to ABAQUS EFTIF values .......................... 87 


\section{LIST OF FIGURES}

Figure 1-1: Latent Heat and Sensible Heat Storage ..................................................... 2

Figure 1-2: Typical variation of effective heat capacity with temperature...................... 7

Figure 3-1: Crossover in Genetic Algorithms........................................................... 34

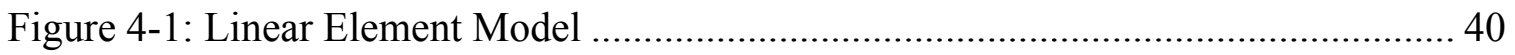

Figure 4-2: Results for select nodal temperatures using linear elements...................... 41

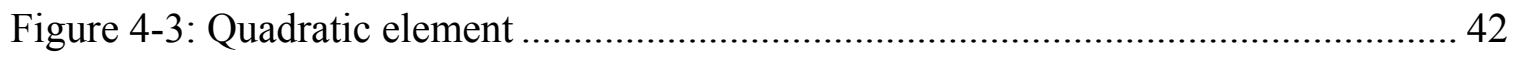

Figure 4-4: Results for select nodal temperatures using quadratic elements .................. 43

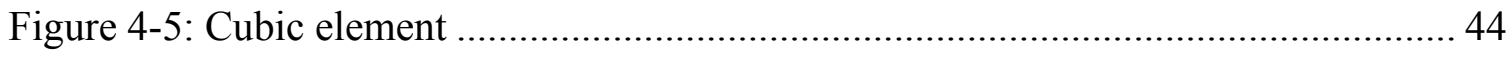

Figure 4-6: Results for select nodal temperatures using cubic elements ....................... 45

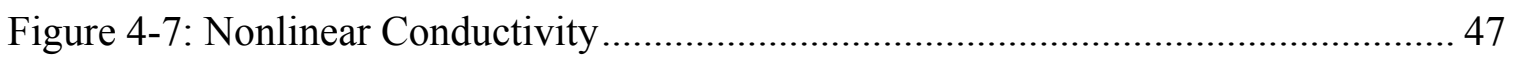

Figure 4-8: Results for nonlinear conductivity using six elements............................. 48

Figure 4-9: Results for nonlinear conductivity using 200 elements ........................... 50

Figure 4-10: Nonlinear latent heat model ............................................................. 51

Figure 4-11: Results for latent heat analysis with 8 elements .................................. 53

Figure 4-12: Results for latent heat analysis with 100 elements ................................ 53

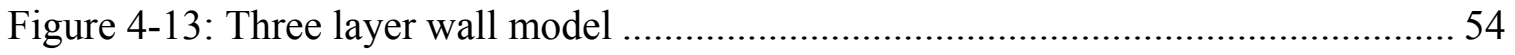

Figure 4-14: (a) Nodal temperature results for the three layer wall model, (b) energy flow

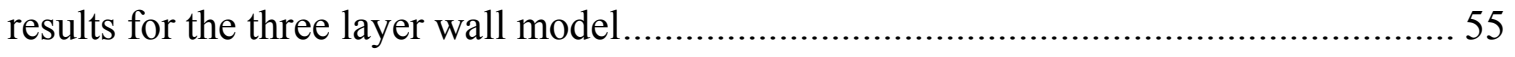

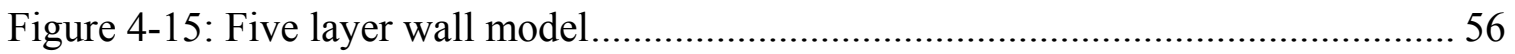

Figure 4-16: (a) Results for nodal temperatures of the five layer wall model, (b) Results

for the energy flow through the five layer wall model ............................................ 56 
Figure 4-17: Wall Design program

Figure 5-1: Ambient air temperature for Phoenix without radiation (a) on June $14^{\text {th }}, 2011$, (b) on January $1^{\text {st }}, 2011$, (c) on March $4^{\text {th }}, 2012$.

Figure 5-2: Ambient air temperature for Phoenix with radiation (a) on June $14^{\text {th }}, 2011$, (b)

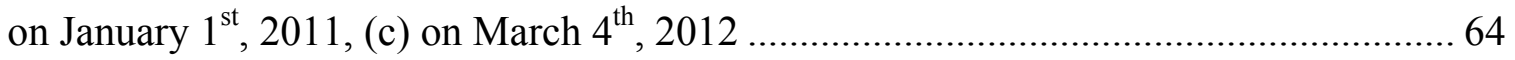

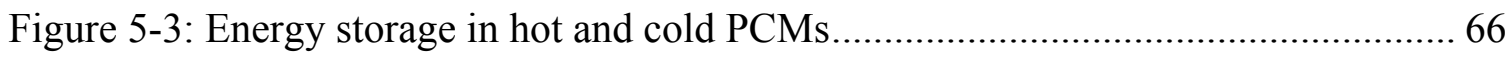

Figure 5-4: (a) 3LW, (b) 5LW (c) 3LW FE model (d) 5LW FE model ......................... 68

Figure 5-5: (a) 3LW structural FE model and (b) 5LW structural FE model................. 71

Figure 5-6: Temperature variation as a function of time on the inside face of the wall (a)

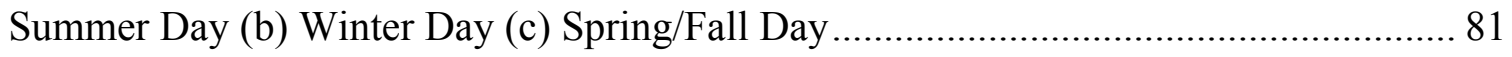

Figure 5-7: Energy flow through the inner face with respect to time (a) Summer Day (b)

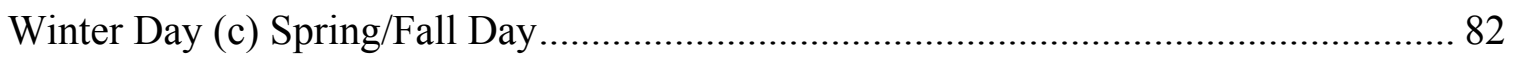

Figure 5-8: Temperature across the wall (a) Normal Concrete Wall, (b) Thickness

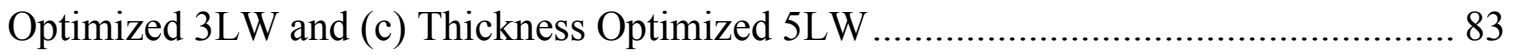

Figure 5-9: PCM Efficiency (a) Summer Day, (b) Winter Day, (c) Spring/Fall Day ...... 84

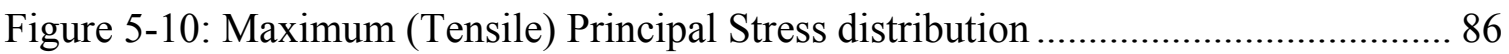




\section{LIST OF SYMBOLS}

\begin{tabular}{|c|c|}
\hline \multicolumn{2}{|c|}{ ABBREVIATIONS } \\
\hline PCM & Phase Change Material \\
\hline TES & Thermal Energy Storage \\
\hline $\mathrm{AZ}$ & Arizona \\
\hline $\mathrm{NC}$ & Normal Concrete \\
\hline HVAC & Heating, Ventilation and Air-Conditioning \\
\hline EFTIF & Energy Flow Though Inside Face \\
\hline PCME & PCM Efficiency \\
\hline $\mathrm{FE} / \mathrm{FEA}$ & Finite Element / Analysis \\
\hline IFAT & Inside Face Air Temperature \\
\hline \multicolumn{2}{|c|}{ NOMENCLATURE } \\
\hline$\rho$ & Density $\left(\mathrm{kg} / \mathrm{m}^{3}\right)$ \\
\hline$c_{p}$ & Specific heat capacity $(\mathrm{J} / \mathrm{kg}-\mathrm{C})$ \\
\hline$c_{p c m}$ & Specific heat capacity in phase change $(\mathrm{J} / \mathrm{kg}-\mathrm{C})$ \\
\hline$k$ & Thermal conductivity $(\mathrm{W} / \mathrm{m}-\mathrm{C})$ \\
\hline$L$ & Latent heat capacity $(\mathrm{J} / \mathrm{kg})$ \\
\hline$T$ & Temperature $(\mathrm{C})$ \\
\hline$T_{L}$ & Liquidus phase change temperature $(\mathrm{C})$ \\
\hline$T_{S}$ & Solidus phase change temperature (C) \\
\hline$t$ & Time $(s)$ \\
\hline$\alpha$ & Solar coefficient of absorption \\
\hline$S$ & Total solar radiation $\left(\mathrm{W} / \mathrm{m}^{2}\right)$ \\
\hline$h$ & Convective co-efficient $\left(\mathrm{W} / \mathrm{m}^{2}-\mathrm{C}\right)$ \\
\hline$\tau$ & Heat flux $\left(\mathrm{W} / \mathrm{m}^{2}\right)$ \\
\hline$A$ & Area $\left(\mathrm{m}^{2}\right)$ \\
\hline$m$ & Mass $(\mathrm{kg})$ \\
\hline$Q_{s}$ & Heat absorbed as sensible energy $(\mathrm{J})$ \\
\hline$Q_{L}$ & Heat absorbed as latent energy $(\mathrm{J})$ \\
\hline$Q_{T}$ & Total heat absorbed (J) \\
\hline$E$ & Energy $(\mathrm{J})$ \\
\hline$q$ & Heat flowing into a system $(\mathrm{J})$ \\
\hline$Q$ & Internal heat source $(\mathrm{J})$ \\
\hline$\vec{l}$ & Length $(\mathrm{m})$ \\
\hline$T_{\infty}$ & Ambient Temperature (C) \\
\hline$T_{0}$ & Initial Temperature $(\mathrm{C})$ \\
\hline
\end{tabular}




\section{INTRODUCTION}

According to the National Science and Technology Council, buildings consume $40 \%$ of the U.S.'s energy consumption and is only continuing to grow with the increase in population [1]. The heating and cooling systems in buildings alone use about $40-50 \%$ of that energy. This energy consumption also has substantial effects on the environment. Nevertheless, these statistics do not have to be so significant and could be greatly reduced by using some of today's new technologies. It is estimated that $30-50 \%$ of building energy consumption could be reduced by integrating such technologies into buildings [1]. The use of these technologies to reduce energy has become one of the biggest modern day challenges and it is up to engineers to create and develop new models to try to provide energy reduction and sustainability for buildings.

There are many new options to reduce this energy problem, but recently phase change materials (PCMs) have become more closely looked at for this purpose. The energy saving benefits that PCMs can provide by integrating them with building elements is significant. This thesis will look at exploring how PCMs work along with developing a modeling technique, including finite element analysis and optimization, to create a computer program to optimize the use of PCM in a wall system in order to reduce the lifetime cost of a building.

\subsection{Phase Change Materials}

Phase change materials, a type of thermal energy storage material (TES); use the physical process of changing form, typically from solid to liquid during a certain temperature range, to store latent energy. This is done by the phase change process where there is very minimal volume change, but huge energy storage capacity. Latent heat is also known as 
phase change enthalpy and heat of fusion, which most materials do not possess [2].

Latent heat storage differs from a materials sensible heat capacity because during phase change, the temperature of melting is kept constant, so the material does not see a change in temperature but is still able to store large amounts of heat. For sensible heat capacity a constant increase in temperature happens to the material while energy is stored [3].

Figure 1-1 represents the difference between sensible heat storage and latent heat storage. As the PCM is melting during its phase change range it is able to absorb heat and store it and once the material transitions back to solid this energy is released back into the environment.

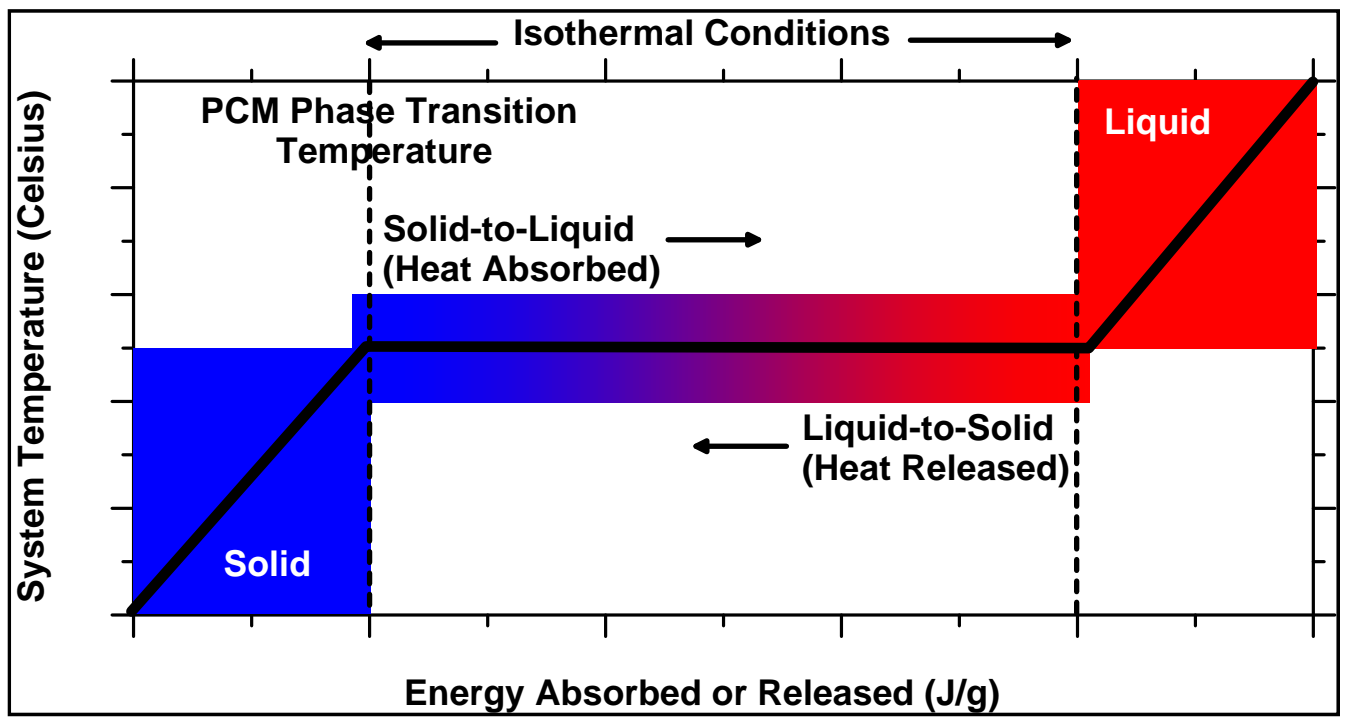

Figure 1-1: Latent Heat and Sensible Heat Storage [4]

There are many types of phase change materials but they all fall in the categories of organic, inorganic or eutectic. Organic PCMs typically have a lower latent heat capacity and melting temperature making them less beneficial as latent heat storage materials. However, they are the safest PCMs to use and they do not segregate, which can be a common problem for PCMs over time. They can also be used with metals. Inorganic PCMs have much larger latent heat storage capacities and a wider range of phase change 
temperatures, but it is difficult to encapsulate inorganic PCMs and their stability during temperature cycling is not as good as organic PCMs. Eutectic water salt solutions are made up of inorganic PCMs, organic PCMs or both, and the combination determines its phase change capacity. The addition of salt into these mixtures lowers the phase change temperature to below freezing, so these are used only for cold climates [2].

The energy storage capacity is the capability that makes PCMs good materials to use for temperature control in a building environment. Unlike typical insulation, PCMs have latent heat capacity so they can reduce the amount of energy that flows across a PCM layer. This keeps both the temperature of the PCM and its surroundings more regulated and allows for the energy to be released from the PCM only when needed, i.e. when the ambient temperature around the PCM has dropped significantly. Due to these benefits there has been much interest in using PCMs in building elements to reduce the large amounts of energy that buildings consume.

There are many studies that look at shifting the heating and cooling loads to off peak electricity periods by using phase change materials. This is a result of energy and heat being stored during peak times and then released during the off peak times, so cooling systems are not needed as much throughout the on peak energy time. This would allow power companies to reduce both their power generation during the peak times and reduce the cost of electricity at all times [5]. With these reductions, there would also be a reduction in the amount of emissions released from these power generating stations because they will not have to work as hard to keep up with the energy demand. The University of South Australia has created a unit using PCMs for heating and cooling that utilizes the off peak power times to charge it up for use during on peak times. The 
University of Nottingham has also developed something similar [6]. A study done by the Center for Energy Research showed that placing a layer of PCM in between two insulation layers in an attic would shift the load times of an air conditioning unit by 11$25 \%$, which is significant over an extended period of time [7]. Along with shifting electricity periods, the use of PCMs has also been studied for being able to stabilize the inside room temperature better than traditional insulation, which eliminates some of the need for an HVAC system altogether. A test with a PCM wallboard was done to confirm that demand from and HVAC unit was reduced for an annual energy savings of about $13 \%$ just by adding a small amount of PCM into the wallboard [8].

The way of incorporating phase change materials into buildings has also been studied extensively. Many of the methods include incorporating PCMs into wallboards like gypsum board $[8,9,10,11,12,13,14]$. Also, incorporating PCMs into different masonry walls like brick or concrete walls has been looked at $[13,15,16,17]$. The methods of including PCMs into these materials are done by impregnation, micro-encapsulation or macro-encapsulation $[13,18,19]$. Impregnation of a PCM material is simply adding the phase change material into a porous medium like gypsum or cement when the material is first being mixed. Micro-encapsulation is the method of enclosing the PCM in a small polymer encasing and macro-encapsulation is done by placing a large amount of PCM in some kind of covering to keep it together in bulk. The studies performed in this project look at the affects of a PCM layer in bulk.

\subsection{Finite Element Analysis used with PCMs}

There are many practical experiments that have been done in labs on PCMs, but finding a way to model and analyze the behavior of this material in an automated way was a main 
goal of this research. The Finite Element Method (FEM) will be looked at as the analysis tool used to study the effects of PCM in a building element. The use of the FEM has been around for many decades as the industry standard to model and solve engineering problems and can be used to solve many different types of problems, such as heat transfer analysis [20]. The FEM transforms an actual system into a model of elements and nodes that can be analyzed, this is called mesh descritization. Since this study looks at temperature and energy regulation, a heat transfer analysis was necessary to perform in order to understand how phase change materials will affect the temperatures in the system. The actual finite element formulation will be discussed in Chapter 2, but there have been many papers and studies done to look at how to apply the FEM to phase change materials. The addition of PCM in a system causes the analysis to become nonlinear. There are many papers that were looked into how to deal with a nonlinear Finite Element Analysis (FEA) [21, 22, 23, 24]. Nonlinearity is the result of a material with a property that is dependent on temperature, which is the case when using PCMs. Once the understanding of nonlinearity was accomplished, there are two different methods that could be applied to the FEA of phase change materials, a fixed mesh approach or a moving mesh approach. The moving mesh approach is also called a moving boundary problem and considers the solid and liquid regions of the PCM as separate regions, while the fixed mesh considers the PCM as a continuous region. The moving boundary problem is typically based on the classical Stefan problem and requires developing deforming grids and meshes which is a complicated process. These also are not accurate for problems where phase change takes place over a temperature range, 
which is the type of PCMs looked at in this study, so only fixed mesh methods are looked into [25].

There are two typical fixed mesh approaches that can be used: the effective heat capacity method and the enthalpy method. The enthalpy method has many different variations that have been previously implemented and used to solve for the temperatures and energy absorption in the system [21]. The enthalpy method allows for the creation of a smooth function using the material's heat capacity and current temperature [25]. The use of the enthalpy method formulation has been discussed extensively [13, 25, 26, 27, 28, 29]. However, the use of the effective heat capacity method does not create a smooth curve but has also been looked at comprehensively in literature [21, 30, 31, 32]. The effective heat capacity is an advantageous method because the addition of a PCM can easily be calculated and integrated into problems where phase change takes place over a temperature range. This is also the method implemented in the finite element analysis program ABAQUS v. 610 [33]. As a result this was the method that was explored and executed in this study. The following figure represents how the effective heat capacity method works, during the phase change range there is a much higher effective heat modeled in the system. 


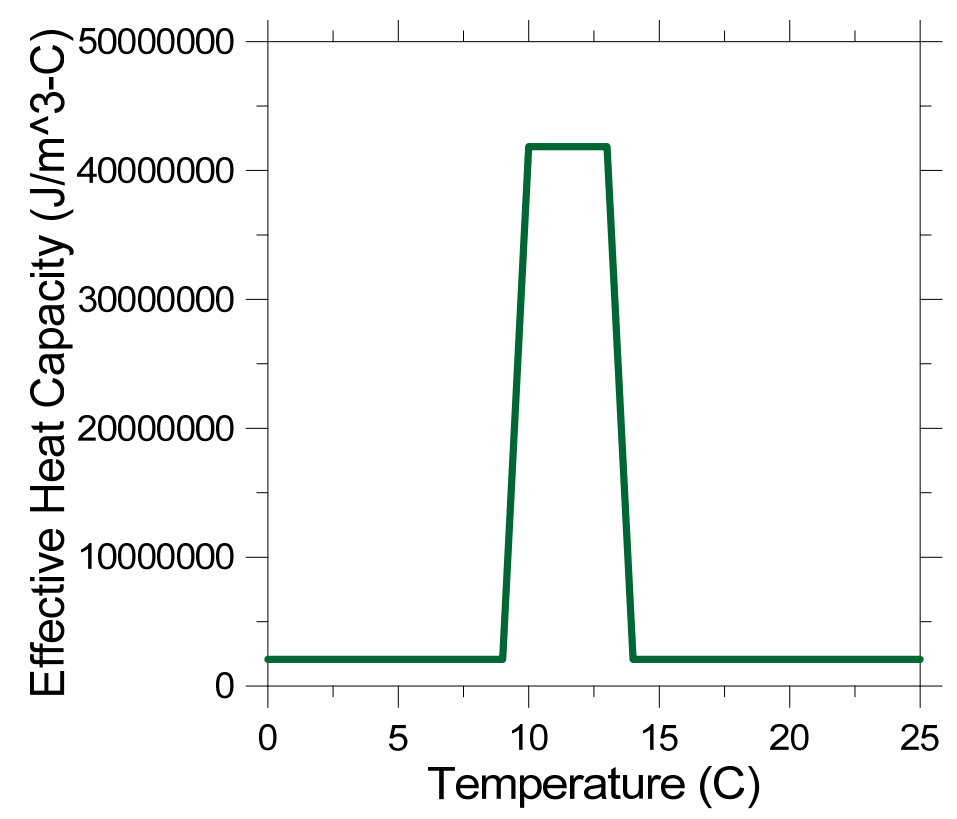

Figure 1-2: Typical variation of effective heat capacity with temperature [21]

\subsection{Optimization and PCMs}

Optimization is the second tool that will be used in this analysis. Optimization has also been around for many years and there are many different methods that can be used [34].

The specific methods used for this study will be discussed in Chapter 3 . There have only been a select few instances where optimization has been applied to walls with phase change materials. In one case, an optimization was done on PCM wallboards using a numerical simulation. This was only an informal optimization using a discrete measure of different wall board thicknesses and studying the amount of energy stored in each wall [12]. In another case an ad-hoc optimization was performed on bulk thicknesses of PCM [35]. This optimization was also based on the amount of energy storage in the PCM system.

However, there is a lack of a proper optimization formulation applied to phase change materials, but the goal of automating the analysis process lends to incorporating an optimizer into the overall program that was developed. There are many variables and 
factors to consider when using phase change materials, which by incorporating a formal optimization there is very little thought that would be needed to produce the best possible results. Also, the cost of using phase change materials in building elements is quite significant, which has hardly been discussed in literature because it is still a new material. While PCMs are a potentially beneficial technology in conjunction with building materials, placing a large amount of PCM in a wall or roof slab could potentially cost more than the consequent energy savings it would provide. As a result, there must be a balance between how much PCM is added to a wall or slab when considering the overall cost of the wall. Thus, cost becomes a significant factor in this study for both material cost and energy cost, which is where optimization will provide a powerful tool.

\subsection{Structural Analysis of Walls with PCM}

These PCM embedded walls will also be studied for their structural integrity. The structural ability of phase change materials have not been well documented and is difficult to quantify as a result of the phase change that the material goes through. Therefore, to perform the structural analysis only the building material, concrete for this study, was considered to provide the structural capacity, so a search for concrete walls or panels with openings was carried out.

One such wall was an insulated wall panel by Hanson, where a layer of insulation was sandwiched by concrete, was studied and designed for ultimate strength and stress tests. The walls are treated as non-composite designs where each concrete layer must resist the loads by itself. The overall testing was based on deflection and stress criteria [36]. Another study was done on precast concrete sandwich panels that consider this system as both composite and noncomposite. The difference is determined by the condition at the 
interface of the two materials. The strains that resulted from the applied lateral loads were found along with the displacements. A linear finite element analysis was also carried out to validate the results, showing that the FEA technique is valid for sandwich type wall systems [37]. Another wall system looked into were waffle walls, which first compute an equivalent thickness for the wall. The waffle walls are tested for buckling capacity because buckling load is assumed to govern these types of walls [38]. An experimental test on concrete sandwich wall panels was done at North Carolina State University. The experiment was performed to track the maximum strains and displacements in the panels. The results show that when the interface between the two materials is stronger, the walls showed higher failure loads. However, it was difficult to create the composite behavior needed with the different types of insulation looked at [39]. All these studies show that creating a composite material when placing an insulating material in between concrete is difficult and that typically the interface between the two materials will govern the amount of load that can be applied.

Furthermore, placing large amounts of PCM in a structural wall member will decrease the overall structural capacity of the member, which also can and should be factored into the optimization framework.

\subsection{Objectives}

This thesis will focus on providing solutions to the following problems.

(1) Effectively analyzing the behavior of phase change materials.

(2) Creating an efficient computer program to automate the process of analysis and optimization to minimize the work needed from a designer. 
(3) Using optimization techniques to create the best possible wall model containing both concrete and PCM.

(4) Using structural analysis to ensure the structural integrity of the wall system remains even with the addition of PCM.

(5) Understanding if a multiple layer PCM wall be more cost effective than a single layer PCM wall.

There are numerous benefits of using phase change materials in buildings elements, but the means of analyzing such a system is complex. Considering both the thermal effects from the PCM along with the structural effects and how those affect the overall cost of a wall system are necessary. The final objective was to create a computer program that has been validated to seamlessly perform the analysis of a PCM embedded wall system. 


\section{FINITE ELEMENT METHODS}

The use of finite element analysis (FEA) for heat transfer problems provides a very powerful numerical method for solving these types of problems. The goal of the FEA approach is to take a differential equation and change it into a set of algebraic equations that can be solved using numerical techniques. There are many different approaches to forming the solution to these problems, but the Weighted Residuals Approach is the technique used for this project. This technique finds an approximate solution and weights it over the problem domain to try to minimize it [20].

Finite element analysis provides a technique to solve both linear and nonlinear problems along with steady state (heat transfer) and transient (diffusion) problems. The method of implementation for all these problem types was explored and implemented in a custom $\mathrm{C}++$ computer program known as the IBVP program. For this program one dimensional FE problems were looked at, which considers discretized linear, quadratic or cubic segments for the element types. For the analysis performed it was assumed that the thermal and mechanical properties of the heat transfer problem are independent; therefore, the change of internal energy is only dependent on temperature and not strains and displacements on the body, so the temperatures of the system become the primary unknown of concern. One of the fundamental ideas used in finite elements is interpolation [20]. An interpolation function is created with shape functions and the nodal values allowing FEA to link the spatial domain of the problem to the element geometry using simple shapes [20]. In this analysis the simple shapes used were one dimensional. Finite elements uses a mapping system between parent and real elements, and the shape function are the interpolated functions created to relate the real element to the parent 
element. The one dimensional parent elements are line segments with length of 2 and coordinates that range from -1 to 1 .

For a one dimensional finite element analysis, the typical element types looked at are $\mathrm{C}^{0}$ linear elements, $\mathrm{C}^{0}$-quadratic elements and $\mathrm{C}^{0}$-cubic elements. As expected, the linear element consists of two nodes, with coordinates of -1 and 1 for the parent element. The quadratic element consists of three nodes with coordinates of $-1,0$ and 1 for the parent element. The cubic element is made up of four nodes; the coordinates are $-1,-1 / 3,1 / 3$ and 1 for the parent element. Each element has the corresponding number of shape functions to nodes that allow the parent elements to map to the real element [40]. If a problem contains more than one element, there must be a set of shape functions derived for each element. These three types of elements are implemented for the linear heat transfer and linear diffusion program, but only the $\mathrm{C}^{0}$-linear element has been incorporated for use in the nonlinear diffusion program.

The principle behind heat transfer analysis follows the principle of conservation of energy, which means the rate of heat energy added to a system must equal the rate of heat energy lost from that system. All heat transfer problems also follow Fourier's law of heat conduction. This constitutive equation is given by:

$$
q(x)=-k(x) \frac{d T(x)}{d x}
$$

From this law and conservation of energy the governing differential equation for heat transfer problems is given by:

$$
-\frac{d}{d x}\left(A(x) k(x) \frac{d T(x)}{d x}\right)+h(x) l(x) T(x)=Q(x) A(x)+h(x) l(x) T_{\infty}
$$

With boundary conditions applied at either end of the system stated by: 
At $x=x_{a}$,

Essential BC: $T=T_{a}$

Natural BC: $q=q_{a}$

Mixed BC: $q=h_{a}\left(T-T_{a}^{\infty}\right)$

The essential boundary condition means at that end the temperature is known. The natural boundary condition considers heat flowing into one end of the system. The mixed boundary condition considers convection taking place, which most closely models the affects of ambient air circulation.

\subsection{Linear Steady State Analysis}

The most basic heat transfer FEA is the linear steady state problem using $\mathrm{C}^{0}$-linear elements; however, these concepts can be expanded for the $\mathrm{C}^{0}$-quadratic elements and $\mathrm{C}^{0}$-cubic elements. The finite element approximation for nodal temperatures of a heat transfer problem are given by:

$\mathbf{T}(\mathbf{x})=\sum_{i} \phi_{i}(\xi) T_{i}$

Where $\phi_{i}$ are the element shape functions based on interpolation. The value of $\mathrm{i}$ ranges the number of nodes an element has. Each shape function must have a value of 1 at the $i$ th node and be zero at all other nodes in an element [41]. For the linear 1D element, which contains two nodes, the corresponding shape functions are: 


$$
\begin{aligned}
& \phi_{1}=l_{0}^{1}=\frac{1-\xi}{2} \\
& \phi_{2}=l_{1}^{1}=\frac{1+\xi}{2}
\end{aligned}
$$

The use of $\xi$ is the coordinate defined and used in the parent element, and it is the value that must be mapped to the actual coordinate system of the real element before the integration of the element equations below can take place. This is done by using isoparametric mapping from the actual element to the parent element. Since the parent element is only integrated over the range $-1 \leq \xi \leq 1$, Gauss-Legendre numerical integration can be used. Gauss-Legendre uses evaluation at sampling points, n, along with a weight application at each point, $w_{j}$. The use of $(\mathrm{n}+1) / 2$ sample points will integrate any polynomial exactly, so it is a very efficient method to use [20]. The transformation to use Gauss-Legendre integration is:

$$
\int_{\Gamma_{e}} n(x) d x=\int_{-1}^{1} N(\xi) d \xi \approx \sum_{k=1}^{n} w_{k} N\left(\xi_{k}\right)
$$

Using these shape functions, the general equation to be solved for any linear steady state problem is $[\mathbf{K}]\{\mathbf{a}\}=\{\mathbf{F}\}$ where

$$
\begin{aligned}
& {[\mathbf{K}]=K_{i j}=\int_{x_{1}}^{x_{2}} \frac{d \phi_{i}(x)}{d x} k(x) \frac{d \phi_{j}(x)}{d x} d x+\int_{x_{1}}^{x_{2}} \phi_{i}(x) \frac{h(x) l}{A} \phi_{j}(x) d x} \\
& \{\mathbf{F}\}=F_{i}=\int_{x_{1}}^{x_{2}}\left(Q+\frac{h(x) l}{A} T_{\infty}\right) \phi_{i}(x) d x-\left[\left(-k(x) \frac{d T}{d x}\right) \phi_{i}(x)\right]_{x_{1}}^{x_{2}}
\end{aligned}
$$

$$
\{\mathbf{a}\}=T_{i}
$$

The " $\mathrm{K}$ " matrix is the stiffness matrix, which for heat transfer problems contains the materials conductivity values. The " $F$ " vector is the load vector and contains all exterior loads applied to the system along with any internal heat sources that act on the system. 
The " $\mathrm{a}$ " vector is the vector that contains the unknowns, which for heat transfer these are the nodal temperatures. Once the integrations have taken place, and assuming that conductivity, $\mathrm{k}$, is constant because this is a linear problem, the element stiffness matrix and force vector can be written as follows:

$$
\begin{aligned}
& {[\mathbf{K}]=\frac{k}{l}\left[\begin{array}{cc}
1 & -1 \\
-1 & 1
\end{array}\right]+h_{1}\left[\begin{array}{ll}
1 & 0 \\
0 & 0
\end{array}\right]+h_{2}\left[\begin{array}{ll}
0 & 0 \\
0 & 1
\end{array}\right]} \\
& \{\mathbf{F}\}=\frac{l}{2}\left\{\begin{array}{l}
Q \\
Q
\end{array}\right\}+\left\{\begin{array}{c}
q_{1} \\
-q_{2}
\end{array}\right\}+\left\{\begin{array}{l}
h_{1} T_{\infty}^{1} \\
h_{2} T_{\infty}^{2}
\end{array}\right\}
\end{aligned}
$$

It is important to note that this is for only a single linear element, but to improve the accuracy of a solution the number of elements used to solve a problem must be increased. This is known as mesh refinement and can be done by breaking a domain of a problem into many different elements. For each element the stiffness matrix and load vector are formed the exact same way. Then the combination of each element's stiffness matrix and force vector forms the entire system matrix equation to solve the entire problem. This system of equations creates a positive definite matrix, which can be solved using many different matrix solvers; the use of Gaussian Elimination was implemented for this project. From the system equation, the primary set of unknowns, the nodal temperatures, can be solved for using $\{\mathbf{a}\}=[\mathbf{K}]^{-1}\{\mathbf{F}\}$. Then the secondary unknown, the heat flux in each element, can then be calculated from the nodal temperatures as:

$$
\tau=-k \frac{d T}{d x}
$$

\subsubsection{Implementation in the IBVP Program}

The development of the IBVP program has been in $\mathrm{C}++$. The overall program algorithm is: 
(1) Read an input file that describes the problem geometry and material data

(2) Solve the FE problem

(3) Compute the Response

(4) Write the Response values to an output file

(5) End the program

In Step 2 is where the actually FEA is performed. Within this function there are three major functions performed:
A. Construct the Stiffness (K) Matrix
B. Construct the Load (F) Vector
C. Impose the boundary conditions on the $\mathrm{K}$ matrix and $\mathrm{F}$ Vector

In function $\mathrm{A}$, the stiffness matrix for the entire model is created by calling another function that applies the numerical integration needed for FEA. This FEA file that is called is where the shape functions are created and mapped to the actual problem for linear, quadratic and cubic elements. Also, the integration order to use for computing the Gauss-Legendre integration is calculated here. Once the shape functions are computed, then back in function A the internal force terms are added to the load vector and the stiffness terms are added to the stiffness matrix. This is performed for each element and then added to the overall global system matrix and global load vector.

In function $\mathrm{B}$, the external loads are added into the global load vector. Then the boundary conditions get imposed in function $\mathrm{C}$. The load vector is adjusted for the boundary conditions along with any terms in the stiffness matrix as needed for essential and mixed boundary conditions. 
Once the stiffness matrix and load vector are created, back in the Solve function (Step 2), the method of Gaussian Elimination is implemented to solve the system of linear equations for the unknown temperature values. The use of Gaussian Elimination is the most efficient way to solve for these values without using a banded storage scheme. Gaussian Elimination does not have to create any new matrices or vectors for storage to solve the system, thus making it more efficient than other system solving techniques. From Step 3, where the response is computed, the element flux is derived from the nodal temperatures that were solved for during Step 2. This function also calls the FEA function file to determine the quadrature points that the flux values need to be calculated at using the Gauss-Legendre rule. Once these are found the results can either be output in text or graphically.

\subsection{Linear Transient Analysis}

Transient problems, also called diffusion or initial boundary value problems (IBVP) are different than steady state problems because the temperature is also a function of time in addition to the spatial variable. Steady state problems rely on knowing the boundary conditions at both sides of the problem; however, for transient problems only the initial condition needs to be stated at the initial time. If time goes to infinity and loads are held steady, then the system will reach equilibrium or its steady state solution. IBVP problems are based on the following differential equation:

$$
c(x) \rho(x) \frac{\partial T(x, t)}{\partial t}-\frac{\partial}{\partial x}\left(k(x) \frac{\partial T(x, t)}{\partial t}\right)+\frac{h(x) l(x) T(x, t)}{A(x)}=Q(x, t)+\frac{h(x) l(x) T_{\infty}}{A(x)}
$$

With initial condition

$T\left(t_{0}\right)=T_{0}$ 
For this problem, temperature now depends on two dimensions, time and space. The spatial dimension is still solved using finite elements and the time variable is solved using finite difference. The problem that now must be solved is $[\mathbf{C}]\left\{\frac{d \mathbf{a}(t)}{d t}\right\}+[\mathbf{K}]\{\mathbf{a}\}=\{\mathbf{F}\}$. The formulation of the additional matrix, known as the capacity matrix, is similar to that of the stiffness matrix, but depends on the sensible heat and the density of the material.

$$
[\mathbf{C}]=C_{i j}=\int_{x_{1}}^{x_{2}} \phi_{i}(x) c(x) \rho(x) \phi_{j}(x) d x
$$

Assuming $\mathrm{c}$ and $\rho$ are constant, once integrated for a $\mathrm{C}^{0}$-linear element the capacity matrix becomes:

$$
[\mathbf{C}]=\frac{\rho c l}{6}\left[\begin{array}{ll}
2 & 1 \\
1 & 2
\end{array}\right]
$$

The time portion of the problem is solved by using a time-stepping method where the time domain is divided into time steps of $\Delta \mathrm{t}$. The nodal temperatures are now calculated at the end of each time step instead of over the continuous time domain [40]. A recurrence relation is used to relate the temperature values at one time step to that of a successive one, thus a linear multistep time-stepping method is used in this analysis. More specifically the $\theta$-method was implemented to compute nodal temperatures at each time step. The $\theta$-method is the most general form of the finite difference methods because $\theta$ can be anything in the range of 0 to 1 during a time step. Where $\theta$ is:

$$
\theta=\frac{t-t_{n-1}}{t_{n}-t_{n-1}}
$$

In order to solve the differential equation at any given time step, the finite difference method is used in order to derive the following equation [40]: 


$$
\left(\frac{1}{\Delta t_{n}}[C]+\theta[K]\right)\{a\}_{n}=(1-\theta)\{F\}_{n-1}+\theta\{F\}_{n}+\left(\frac{1}{\Delta t_{n}}[C]-(1-\theta)[K]\right)\{a\}_{n-1}
$$

When $\theta=0$, this becomes the forward difference method

$\theta=1 / 2$, this becomes the mid difference method

$\theta=1$, this becomes the backward difference method.

This can be simplified into the typical FEA equation with the form of:

$$
\left[\mathbf{K}_{\text {eff }}\right]\{\mathbf{a}\}=\left\{\mathbf{F}_{\text {eff }}\right\}
$$

Where

$$
\begin{aligned}
& {\left[\mathbf{K}_{\text {eff }}\right]=\left(\frac{1}{\Delta t_{n}}[C]+\theta[K]\right)} \\
& {\left[\mathbf{F}_{\text {eff }}\right]=(1-\theta)\{F\}_{n-1}+\theta\{F\}_{n}+\left(\frac{1}{\Delta t_{n}}[C]-(1-\theta)[K]\right)\{a\}_{n-1}}
\end{aligned}
$$

The capacity and stiffness matrices and load vector are formulated the same way as before using equations $2.7,2.8$, and 2.14 for every time step in order to solve for the nodal temperatures at the time of interest. In order to use this method the current force vector and previous force vector must be known along with the previous vector of nodal temperatures.

\subsubsection{Implementation into the IBVP Program}

The main program is the same as for solving a steady state problem; however, in Step 2 an overall loop through the time domain of the problem was implemented. The program was developed to allow the use of different time increments which can have varying number of steps, $\Delta \mathrm{t}$ and $\theta$ values between each increment. As a result, within Step 2, where the problem is solved, the same functions A-C are still performed, but within the 
loops of the intervals and the number of time steps within each interval. The overall time loop is:

(2.1) Loop through all time intervals

(2.2) Loop through all time steps in each interval

A. Construct the Stiffness (K) Matrix

B. Construct the Load (F) Vector

C. Impose the boundary conditions on the $\mathrm{K}$ matrix and $\mathrm{F}$ Vector

(2.3) End time step loop

(2.4) End interval loop

The functions A, B and C were also updated to become a function of the time interval and the steps within the interval because the previous and current values for the load vector and the previous values for the temperature vector must be stored. The capability to make the capacity matrix had to be added within function A and in the FEA function that is called because shape functions are used to create the capacity matrix. Also, within function $\mathrm{A}$, the $\mathrm{K}_{\text {eff }}$ matrix is now created and stored as the global stiffness vector. Then back in the overall solve function the $F_{\text {eff }}$ is created based on the value of $\theta$ specified for the interval. Again, Gauss Elimination is used in solving for the nodal temperatures of each increment. The loop is continued until all the intervals and time steps have been computed.

For this type of problem the load vector and temperature vectors are no longer vectors, but stored as matrices the size of the time domain. This allows all the temperatures to be computed and stored within the solve function, Step 2, and then when the response is computed in Step 3 the element flux at every time step can be computed from the stored 
nodal temperature matrix and output for every node. The overall loop within the time domain was also implemented in the Step 3.

\subsection{Nonlinear Transient Analysis}

There are several components of a heat transfer problems that can cause nonlinearity, such as temperature dependent material properties. This was the issue explored and implemented in this analysis. Two different cases were looked at, where conductivity is a function of temperature and materials that have latent heat such as phase change materials.

\subsubsection{Temperature Dependent Conductivity}

When conductivity changes with temperature it becomes a challenge to isolate the unknown temperature because the stiffness matrix becomes dependent on temperature too. The governing equation cannot be solved by exclusively using FEA. Newton's method was incorporated to solve the nonlinear portion of the problem. Within the time stepping method this iterative process was added in. Newton's method is an approximation method used to find the roots of a real function by starting each Newton's loop with an initial guess of the unknowns, in this case the initial guess is always the previous values of the nodal temperatures. Once this is computed a better approximation can be made using

$$
x^{i+1}=x^{i}-\frac{f\left(x^{i}\right)}{f^{\prime}\left(x^{i}\right)}
$$

Since this is a system of equations the derivative of the function is computed by finding the residual error in the system. The conductivity depends on temperature, so the initial temperature value given is used to compute the stiffness matrix initially and solve the following equation for the new temperatures: 
$[\mathbf{K}]\left\{\mathbf{a}^{i}\right\}=\{\mathbf{F}\}$

These new nodal temperatures found at this point are based on the initial guess along with the initial conductivity matrix, so computing the residual for this step determines how accurate the initial guess was.

$$
\{\mathbf{R}\}=[\mathbf{K}]\left\{\mathbf{a}^{i}\right\}-\{\mathbf{F}\} \leq \text { tolerence }
$$

The goal of Newton's method is to minimize the amount of error in the residual, so an iterative approach is ensued. A new system of equations is solved for, instead of finding the new temperatures, the amount to adjust the temperatures based on the residual are now calculated instead. The stiffness matrix must be recomputed for each iteration depending on the new temperatures found and then this system of equations is used to update the temperature vector:

$$
[\mathbf{K}]\{\Delta \mathbf{a}\}=\{\mathbf{R}\}
$$

From solving the above equation the change in the temperature values are found and the new temperature values can be calculated by the following:

$$
\left\{\mathbf{a}^{i+1}\right\}=\left\{\mathbf{a}^{i}\right\}+\{\Delta \mathbf{a}\}
$$

Once the convergence tolerance is satisfied, the Newton's loop is complete, and the new nodal temperatures for that time step are found and the program moves on to the next time step.

\subsubsection{Implementation into the IBVP program}

Small updates were made to the IBVP program to allow the capability to compute temperature dependent conductivity problems. A new function to compute the Stiffness Matrix for nonlinear analysis was created, where the current element temperature is computed as the average of the nodal temperatures. The conductivity is then calculated 
and used based on this value. Within the time step loop (Step 2.2) an iteration loop was added in to perform Newton's method. The $\mathrm{K}_{\mathrm{eff}}$ and $\mathrm{F}_{\text {eff }}$ vectors are still computed the same way, but are based on the average element temperature (initially determined by the initial guess values). However, before Gaussian elimination is performed the following loop is implemented:

(2.2.1) Loop through the degrees of freedom in the problem

(2.2.2) Compute the residual of the system

(2.2.3) Perform Gaussian Elimination for equation 2.24

(2.2.4) Update the temperature values

(2.2.5) Check against the convergence tolerance

(2.2.6) Once convergence is reached break the Newton loop

Once the solution converges, the program moves onto the next time step and a new Newton's loop is started to solve for the temperatures in the new step.

\subsubsection{Latent Heat}

Latent heat comes from phase change materials, which have higher energy storage capacity during the materials phase change temperature range. During this temperature range, the material changes from solid to liquid and can store energy at a much higher rate as a result of this phase change. Once the material changes back to solid, the stored energy is released back into the environment at the same higher rate. The addition of latent heat causes the problem to be nonlinear because the capacity matrix is now dependent on temperature. Since the capacity matrix for linear problems is made of the sensible heat capacity and the density of the material, for these types of problems the latent heat capacity must be added in during the materials phase change range. As a result 
there are three different situations that must be considered, (1) the element is out of phase change range, (2) the element is in the phase change range or (3) the element is transitioning either into or out of the phase change range. FEA can still be used to solve this system; however, during a transitions period an iterative approach must be done to ensure the residual of the system has converged. The following equations are solved assuming $\theta=1$, since this is the value that the ABAQUS FEA software uses, so all test cases discussed in this paper using latent heat assume this value of theta.

Each situation must be considered at the element level and not the system level. If an element is in transition or in phase change that does not mean the elements surrounding it are in the same situation. The necessary additions must be made at the system level but only corresponding to the elements that are in phase change or transition.

The first situation to consider is when the element is out of phase change. This is the standard diffusion problem and is solved in the same manner as Section 2.2. The overall equation is the same as equation 2.17 with $\theta=1$, but will be put in a different form to understand how the addition of latent heat works.

$$
\frac{1}{\Delta t_{n}}[C]\left(\{a\}_{n}-\{a\}_{n-1}\right)+[K]\{a\}_{n}=\{F\}_{n}
$$

Where the capacity matrix is still made up of the sensible heat capacity and density only. The second situation to consider is when a problem is in the phase change range. Once an element is in the phase change range, the latent heat capacity must be added into the capacity matrix by the effective heat capacity method. For this program the latent heat capacity was taken as: 


$$
c_{p c}=\frac{L}{T_{L}-T_{S}}
$$

In phase change, the additional part of the capacity matrix is created by:

$$
[\mathbf{C}]_{P C}=\frac{\rho c_{p c} l}{6}\left[\begin{array}{ll}
2 & 1 \\
1 & 2
\end{array}\right]
$$

This is added to the sensible heat capacity matrix to create the new equation to be solved when in phase change, which is:

$$
\frac{1}{\Delta t_{n}}\left([C]+[C]_{P C}\right)\left(\{a\}_{n}-\{a\}_{n-1}\right)+[K]\{a\}_{n}=\{F\}_{n}
$$

The final situation to consider is when an element is transitioning into or out of phase change. This situation results in two different equations, one for transitioning into phase change range and one for transitioning out of phase change range. For both the transition in and transition out of phase change there are two additional situations to consider, (1a) if the element is transitioning from below the solidus temperature into phase change or (1b) from above the liquidus temperature into phase change and (2a) if the element is transitioning out of phase change to solid form or (2b) if the element is transitioning out to liquid form. The overall equation is very similar for both types of transitions in and out of phase change. The first equation represents the transition into phase change from solid form:

$$
\frac{1}{\Delta t_{n}}[C]\left(\{a\}_{n}-\{a\}_{n-1}\right)+\frac{1}{\Delta t_{n}}[C]_{P C}\left(\{a\}_{n}-\left\{T_{S}\right\}\right)+[K]\{a\}_{n}=\{F\}_{n}
$$

When an element transitions in from liquid form the liquidus temperature must be used instead, as given by the following equation: 


$$
\frac{1}{\Delta t_{n}}[C]\left(\{a\}_{n}-\{a\}_{n-1}\right)+\frac{1}{\Delta t_{n}}[C]_{P C}\left(\{a\}_{n}-\left\{T_{L}\right\}\right)+[K]\{a\}_{n}=\{F\}_{n}
$$

However, when an element transitions out of phase change the equation is adjusted.

When the element transitions out of phase change to below the solidus temperature the following equation applies:

$$
\frac{1}{\Delta t_{n}}[C]\left(\{a\}_{n}-\{a\}_{n-1}\right)+\frac{1}{\Delta t_{n}}[C]_{P C}\left(\left\{T_{S}\right\}-\{a\}_{n-1}\right)+[K]\{a\}_{n}=\{F\}_{n}
$$

When the element transitions out to above the liquidus temperature the equation is as follows:

$$
\frac{1}{\Delta t_{n}}[C]\left(\{a\}_{n}-\{a\}_{n-1}\right)+\frac{1}{\Delta t_{n}}[C]_{P C}\left(\left\{T_{L}\right\}-\{a\}_{n-1}\right)+[K]\{a\}_{n}=\{F\}_{n}
$$

For each of the above equations the components that make up $\mathrm{K}_{\text {eff }}$ are every matrix that is multiplied by $\{a\}_{n}$, the vector of unknowns, and everything else makes up the $\mathrm{F}_{\text {eff }}$ vector. During this transition phase, an iterative technique must be used when a model contains multiple elements. Once one element transitions into phase change range, this will affect the nodal temperatures of that element which will then affect the nodal temperatures of the surrounding elements. As a result, the residual is calculated for each iteration and once it converges then the next time step can be solved for.

For the situations when the element is transitioning into the phase change range, this could be either heating up or cooling down, the nodal temperatures are first solved for using equation 2.26, which does not consider any latent heat capacity. Then the average temperature of the element is computed based on the nodal temperatures found, and if the element temperature is within the phase change range this element is considered to be in transition. Once an element is in transition, the iterative process is started and the new 
nodal temperatures are computed using equation 2.30 or 2.31 . The residual of the entire system can then be calculated as:

$$
\|\{\mathrm{R}\}\|=\{F\}_{n}+\frac{1}{\Delta t_{n}}[C]\{a\}_{n-1}+\frac{1}{\Delta t_{n}}[C]_{P C}\left\{T_{S}\right\}-\left(\frac{1}{\Delta t_{n}}\left[[C]+[C]_{P C}+[K]\right]\right)\{a\}_{n} \leq \text { tolerance }
$$

Or

$$
\|\{\mathrm{R}\}\|=\{F\}_{n}+\frac{1}{\Delta t_{n}}[C]\{a\}_{n-1}+\frac{1}{\Delta t_{n}}[C]_{P C}\left\{T_{L}\right\}-\left(\frac{1}{\Delta t_{n}}\left[[C]+[C]_{P C}+[K]\right]\right)\{a\}_{n} \leq \text { tolerance }
$$

This is iterated through until the solution converges. Once an element has transitioned into phase change for the next time step it is considered in phase change until it transitions out and equation 2.29 is used to solve for the nodal temperatures.

When an element transitions out, the current nodal temperatures are first solved using equation 2.29, which considers latent heat capacity. If the new element temperature is found to be outside the phase change range then the element is considered to be transitioning out and the same type of iterative process is used, but equation 2.32 or 2.33 is used to solve for the nodal temperatures. A residual must be calculated to ensure that the solution is converging.

$$
\|\{\mathrm{R}\}\|=\{F\}_{n}+\frac{1}{\Delta t_{n}}\left([C]+[C]_{P C}\right)\{a\}_{n-1}-\frac{1}{\Delta t_{n}}[C]_{P C}\left\{T_{S}\right\}-\left(\frac{1}{\Delta t_{n}}[[C]+[K]]\right)\{a\}_{n} \leq \text { tol }
$$

$\mathrm{Or}$

$$
\|\{\mathrm{R}\}\|=\{F\}_{n}+\frac{1}{\Delta t_{n}}\left([C]+[C]_{P C}\right)\{a\}_{n-1}-\frac{1}{\Delta t_{n}}[C]_{P C}\left\{T_{L}\right\}-\left(\frac{1}{\Delta t_{n}}[[C]+[K]]\right)\{a\}_{n} \leq \text { tol }
$$

Once the entire system has converged the element is considered outside of the phase change range for the following time steps and equation 2.26 is used until the element transitions back into phase change. 


\subsubsection{Implementation into the IBVP program}

The incorporation of latent heat into the program was essential because phase change materials were the main focus of this overall project. The major adjustments came from determining which state an element was in if the material had latent heat capacity. Dealing with an element out of phase change was done exactly the same way as in Section 2.2, but dealing with an element in phase change, the latent heat capacity had to be added to the capacity matrix. The major program adjustment was to determine if an element was in transition and was added into the program in Step 2. This step still solves for the new nodal temperatures the same way as previously using either equation 2.26 or 2.29, but the old nodal temperatures from the previous time step are also stored at that time. Then the average element temperature is computed for both the new time step (and stored in a new element temperature vector) and for the old time step (and stored in a history temperature vector) and checked against a series of "if" statements to determine the elements state. The if statements are as follows:

- If the NewTemp $>T_{S}$ and the HistoryTemp $<T_{S}$, the element is transitioning into phase change.

- If the NewTemp $<T_{S}$ and the HistoryTemp $<T_{S}$, the element is out of phase change.

- If the $T_{S}<$ NewTemp $<T_{L}$ and the $T_{S}<$ HistoryTemp $<T_{L}$, the element is in phase change.

- If the NewTemp $>T_{L}$ and the HistoryTemp $<T_{L}$, the element is transitioning out of phase change. 
- If the NewTemp $>T_{L}$ and the HistoryTemp $>T_{L}$, the element is out of phase change.

- If the NewTemp $<T_{L}$ and the HistoryTemp $>T_{L}$, the element is transitioning into phase change.

- If the NewTemp $<T_{S}$ and the HistoryTemp $>T_{S}$, the element is transitioning out of phase change.

If a single element is in the transition stage, there are four different scenarios to cause this, and then an iterative technique is started. Within this loop, the stiffness and capacity matrix are recomputed based on the situation the element is in and new nodal temperatures are calculated. A residual is computed for this transition time step and once the residual has converged, the iterative loop is broken and the program moves to the next time step. 


\section{OPTIMIZATION METHODS}

The purpose of optimization is to find the best design for a given problem typically dependent on constraints. There are many different types of optimization methods and ways to implement optimization techniques. Since one of the overall purposes of this project was to build a computer program to perform the computations, the optimization methods looked at were best suited for computer integration. There are two main types of search methods used for optimization, a point search or a population search, and for this study population search methods were explored.

The goal of any optimization is to minimize an objective function subject to design constraints. The objective function can be either a cost or weight value. The values that are being manipulated to minimize the objective function are the design variables, which can be either continuous or discrete values depending on the design. The constraints can be either equality or inequality constraints. This program only looks at the use of inequality constraints, and has the ability to deal with both discrete and continuous design variables. The typical optimization problem can be stated as follows [34]: 
Find $\quad \mathbf{x}=\left\{\begin{array}{c}x_{1} \\ x_{2} \\ \vdots \\ x_{n}\end{array}\right\}$

To minimize $f(\mathbf{x})$

Subject to

$$
\begin{array}{ll}
g_{i}(\mathbf{x}) \leq 0 & i=1,2, \ldots, m \\
h_{j}(\mathbf{x})=0 & j=1,2, \ldots, p
\end{array}
$$

For this project, two types of Evolutionary Algorithms (EAs) optimizers were looked at for their techniques. EAs are becoming more popular in optimization because they are well suited for multi-objective functions and not based on the gradient of the function like many other methods, so discrete design variables can be used. They provide the ability to search for multiple solutions in parallel and can handle problems with discontinuities making them efficient optimizers to use [42]. The two EA methods looked at were Genetic Algorithms (GA) and Differential Evolution (DE). These both perform population searches to find an optimum value and are based on the same principles of evolution such as reproduction, mutation and selection.

\subsection{Genetic Algorithms}

Genetic Algorithms is based on natural genetics and selection to perform its search for the optimum point, it is often compared to Darwin's Theory of Evolution [34]. GA can be applied to problems where both continuous and discrete variables are being used because it uses binary strings as variables and it is not necessary to compute the gradient of the function, making it very applicable to this project. 
The optimization problem that will be looked at in this study is a minimization problem subject to several constraints. However, GA optimizes unconstrained maximization problems by evaluating a fitness function. Therefore, the optimization problem must be converted into an unconstrained maximization problem by applying penalty functions into the objective function for the constraint values. This transformation looks like:

To minimize $\quad \beta(\mathbf{x})=f(\mathbf{x})+\sum_{i=1}^{m} r_{i}\left(g_{i}(\mathbf{x})\right)^{2}+\sum_{j=1}^{p} r_{j}\left(h_{j}(\mathbf{x})\right)^{2}$

Where $r_{i}$ and $r_{j}$ are the penalty functions applied to the constraints. Then this new unconstrained objective function must be turned into a maximization problem by:

$$
F(\mathbf{x})=\frac{1}{1+\beta(\mathbf{x})}
$$

This is now the fitness function that GA will try to optimize. As equation 3.4 shows, only penalty will be added to the objective function if a constraint is violated and the square of that violated amount will be added. Otherwise the fitness function is not affected by the penalty function if there is no violation [34].

To start GA, each design variable is transformed into a binary string, known as a chromosome. All the chromosomes from the initial design variables are strung together to create a design vector. Random selection and crossover are used to create a population containing many more design vectors based on the initial starting design vector and the bounds of the problem. The size of the population of design vectors can be varied based on the user's desired accuracy and computation time. Each design vector is then evaluated at the fitness function. Once each design vector in a population has been evaluated, a new set of design vectors can be created for the next generation. The new design vectors are created by reproduction, crossover and mutation to create the new 
chromosomes. This is done until an entire new population has been created and then this new population of design vectors are evaluated to create the new generation. This process is repeated until the maximum number of generations has been performed.

The three major operations of GA are reproduction, crossover and mutation. Reproduction is implemented to select chromosomes from the previous generation to use to create the new generation. Typically four parent chromosomes are selected and competitive selection is used to select the two best parent chromosomes to use for the creation of the child chromosome. Then crossover is executed to exchange pieces of the parent chromosomes with each other to form the new chromosome. Single point crossover is used to pick a point in the binary strings to swap between the two chromosomes. The crossover probability is set to determine how many chromosomes will actually mate and crossover; therefore, some of the previous chromosomes will carry through to the next generation. The final operation is mutation, which typically creates chromosomes with better fitness functions. Mutation changes part of a chromosome from 0 's to 1's or from 1's to 0 's based on the mutation probability set. This probability determines the amount of bits that will actually be flipped for a chromosome. The use of these three procedures allows the creation of a better generation of chromosomes which provides better fitness functions, to ultimately find an optimal point if enough generations are performed. As a result any unacceptable design vector will not survive through to the next generation [34]. 


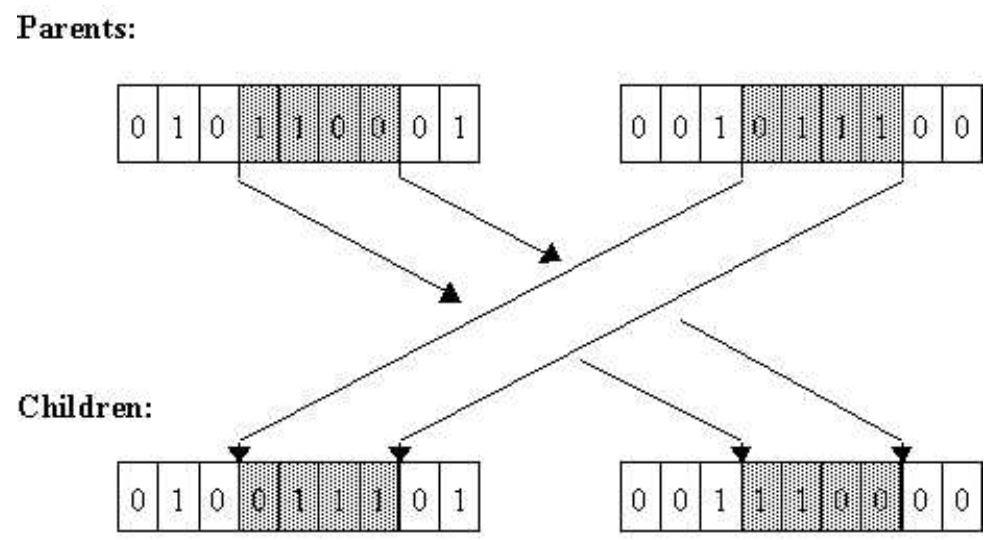

Figure 3-1: Crossover in Genetic Algorithms

\subsection{Differential Evolution}

Differential Evolution is similar to GA and based on mutation, selection and crossover as well. However, DE uses numerical values to create design vectors instead of binary code [43]. An initial population is created in a similar manner to that of GA but instead contains numerical vectors. This initial population of vectors is evaluated at the fitness function, and then mutation, crossover and selection begins.

The way the vectors are manipulated is where DE differs. For DE, three design vectors are chosen from the population and the weighted difference of two of those vectors are added to the third to create a new vector, this is known as the mutation phase.

$$
v_{i}=x_{1}+F\left(x_{2}-x_{3}\right)
$$

Then this new vector is crossed over with a fourth vector to create the final new design vector. Selection plays out by computing the fitness functions of the new vector and the fourth vector that it was crossed over with, and whichever vector results in the lower objective value is selected for the next generation. This is repeated until a new population 
of design vectors has been created for the next generation, and finally stops once the maximum number of generations has been reached [ 43, 44].

These two optimization techniques are fairly similar, but it has been stated that Differential Evolution typically converges faster [42]. This is the result of the mutation and selection of multiple design vectors at once instead of only two binary vectors. However, Genetic Algorithms can have a quicker computation time due to simpler calculations by using binary and can be more computationally efficient when performing a large number of generations with a large population size. As a result, both optimizers were implemented into the program for the user to decide which one better suits the problem.

\subsection{Implementation into the WallDesign Program}

The capability to optimize a design was implemented into a custom $\mathrm{C}++$ program known as the WallDesign program. The actual optimizers were developed prior to this project as a separate optimization toolbox packaged known as the Engineering Design Optimization (EDO) Suite and was integrated into the WallDesign program as a toolbox [45]. Since this project will focus on a wall design where both continuous and discrete variables will be used, GA and DE were the best choices for optimizers to use from the EDO Suite. The additional functions for the optimization were placed in the main computing function in the WallDesign program. However, there were only certain functions from EDO that had to be modified and updated to work within the WallDesign program. The overall optimization algorithm follows:

(1) Set the optimization method and the number of continuous and discrete design variables 
(2) Set the initial values, bounds and precision for these variables

(3) Create the initial population of design vectors

(4) Compute the fitness function for each member in a population

(5) Update the design values to create the next generation

(6) Repeat from Step 4 until the maximum number of generations has been performed The capability to define what the design variables actually are and if they are continuous or discrete was developed to allow the designer to input the parameters in an input file along with the optimization method. The design variables are defined with initial values and bounds. The precision value is preset, but can be updated, and it determines the length of the chromosomes or design vectors created depending on the optimization method used. Step 2 and 3 then take the information input by the user to create the entire first population to be optimized.

In Step 4, which defines the objective function and the constraint values and computes these values is the most important part of the optimizing process. The user has the capability to define the cost values used in the objective function along with all the constraint values to be used. The computation of the constraint values determines if there is a violation of the design and is used when creating the next generation of design vectors. Then the design variables are updated in Step 5 to move onto the next generation of the optimization.

Steps 2-6 are all perfomed within the WallDesign function; however, the main program is where Step 1 is done to select the optimizer type along with the number of design variables. The overall procedure for both GA and DE are the same, they just differ in the way the new generations are formed. The were certain parameters preset and used for the 
tests perfomed in this thesis. For Genetic Algorithms, the penalty parameter used to compute the fitness function is automatically computed, while the crossover probabilty is set to 0.8 and the mutation probability is set to 0.03 For Differential Evolution the value of crossover was set to 0.9 , and the selection strategy was set to random and to choose the best of those to manipulate. These parameters can be changed for future tests based on user preference, to learn how refer to Engineering Design Optimization Workbench User's Manual [45]. 


\section{VALIDATION OF PROGRAM}

The interest in using PCM as an insulating layer in a wall became the motivation for finding a way to quickly and effectively analyze such a system. Finite elements were selected as the best method to do the analysis. The mechanized process of using finite elements allowed for the entire analysis to become computer automated. Once analysis could be done by the computer, incorporating several other capabilities for the wall design program became available. The use of optimization, a tedious task, could also be integrated into the computer program, so not only can a wall design be analyzed but it can be optimized to provide the best design for an engineer based on the given constraints. The task of generating usable and warranted output could also be automated. As a result, the overall goal and ultimate product became a computer program to analyze and optimize phase change materials in a wall made of typical building materials, like concrete, to see how energy flow through wall can change.

These important pieces of the wall design system were developed in $\mathrm{C}++$ through two different computer programs. The programs are an IBVP program and a WallDesign program. The IBVP program contains the ability to perform the finite element analysis on linear and nonlinear steady state and transient problems. There are two key values that are computed in the IBVP program, the nodal temperatures (T) and the heat flux $(\tau)$ in the element. The WallDesign program contains the ability to post process these results to create output and the capability to optimize the wall design. The WallDesign program was developed initially to work with ABAQUS as the FEA software, the results and implementation of this program has been documented extensively in [35]. However, developing this further into a self contained program came with the development and 
integration with the IBVP program. As with any product, the IBVP program had to be validated. The initial stage of developing the IBVP program was the linear transient analysis. The solutions to several problems were compared against the results of David Burnett's program UNAFEM [40]. Since the UNAFEM program does not deal with nonlinearity, the results for nonlinear transient analysis was justified by comparisons with ABAQUS v. 6.10 [33]. Once the IBVP program was validated it was incorporated with the WallDesign program. The addition of the optimization capability was included directly in the WallDesign program itself, but the use of this tool has also been validated.

\subsection{IBVP linear transient analysis}

The UNAFEM program is based on the $\theta$-method of diffusion problems, which was the desired method for the IBVP program. The use of the $\theta$-method allows the user to specify different time intervals with different theta values, depending on the desired stability.

Also, for the different time intervals there could be changes in the boundary conditions or forces applied to the system so these all had to be considered [40]. There were many test cases ran to ensure that the results from the IBVP program and UNAFEM matched. The linear, quadratic and cubic elements were all developed and tested at this stage for the IBVP program. A sample comparison of each can be seen in the following figures and table.

\subsubsection{Linear Element}

This test uses multiple elements with different internal heat sources in each element. The left end is insulated and the right end is kept at a temperature of $10^{\circ} \mathrm{C}$ for 20 seconds and then changes to $8^{\circ} \mathrm{C}$ for the remaining time. Also varying number of time steps were used 
in each time interval, with changing $\Delta \mathrm{t}$ values and changing $\theta$-values for each. Figure 4-1 below shows the geometry and parameters for the problem.

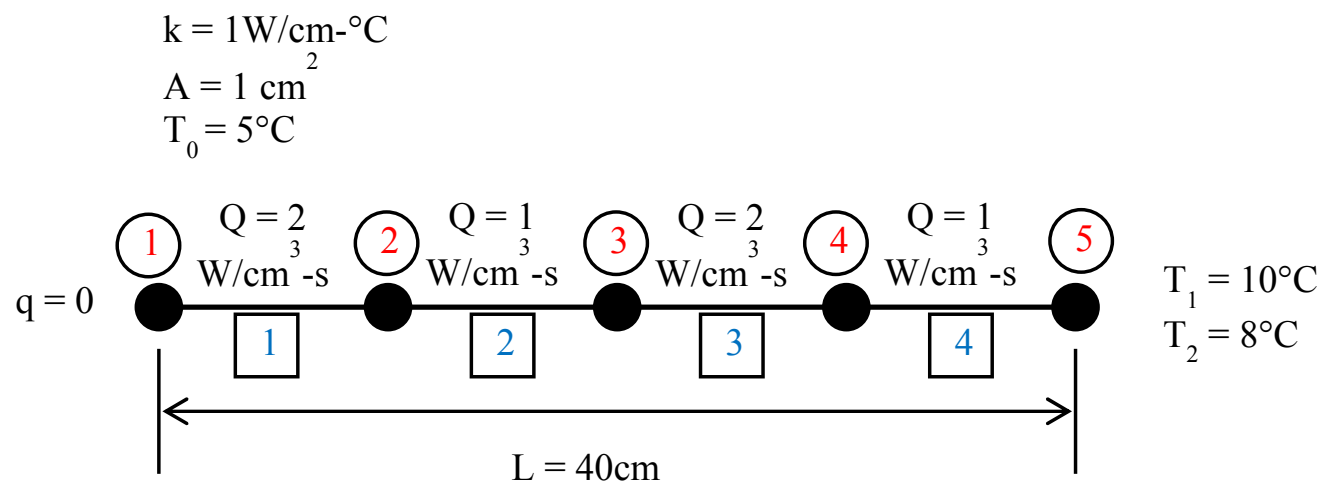

Figure 4-1: Linear Element Model

The following tables show the time step data followed by the results for the linear element test. The figure represents the results for both programs nodal temperature values at node 1 and node 3 . As these results show the IBVP program and the UNAFEM program have matching results when using linear elements.

Table 4-1: Time Step Data for Linear Element

\begin{tabular}{|c|c|c|c|c|c|}
\hline Interval & $\begin{array}{c}\text { Number of } \\
\text { Time Steps }\end{array}$ & $\Delta \mathbf{t}(\mathbf{s})$ & $\theta$-Value & $\begin{array}{c}\text { Left End } \\
\text { BC }\end{array}$ & $\begin{array}{c}\text { Right End } \\
\text { BC }\end{array}$ \\
\hline 1 & 1 & 10 & 1.00 & NBC 0 & EBC 10 \\
\hline 2 & 2 & 5 & 0.4 & NBC 0 & EBC 10 \\
\hline 3 & 5 & 3 & 0.67 & NBC 0 & EBC 8 \\
\hline 4 & 2 & 10 & 0.33 & NBC 0 & EBC 8 \\
\hline
\end{tabular}


Table 4-2: Results for Linear Element

\begin{tabular}{|c|c|c|c|c|}
\hline Time Interval & Time (s) & Node & $\begin{array}{c}\text { IBVP Nodal } \\
\text { Temperature } \\
\left({ }^{\circ} \mathbf{C}\right)\end{array}$ & $\begin{array}{c}\text { UNAFEM Nodal } \\
\text { Temperature } \\
\left({ }^{\circ} \mathbf{C}\right)\end{array}$ \\
\hline 1 & 10 & 1 & 24.99 & 25 \\
\hline 1 & 10 & 2 & 25.01 & 25.01 \\
\hline 1 & 10 & 3 & 24.85 & 24.85 \\
\hline 1 & 10 & 4 & 26.93 & 26.93 \\
\hline 1 & 10 & 5 & 10 & 10 \\
\hline 2 & 20 & 1 & 45.02 & 45.02 \\
\hline 2 & 20 & 2 & 45.01 & 45.01 \\
\hline 2 & 20 & 3 & 44.51 & 44.51 \\
\hline 2 & 20 & 4 & 51.46 & 51.47 \\
\hline 2 & 20 & 5 & 10 & 10 \\
\hline 3 & 35 & 1 & 75.05 & 75.07 \\
\hline 3 & 35 & 2 & 74.74 & 74.74 \\
\hline 3 & 35 & 3 & 75.96 & 75.9 \\
\hline 3 & 35 & 4 & 82.52 & 83.36 \\
\hline 3 & 35 & 5 & 8 & 8 \\
\hline 4 & 55 & 1 & 114.74 & 114.7 \\
\hline 4 & 55 & 2 & 115.07 & 115.1 \\
\hline 4 & 55 & 3 & 118.35 & 118.5 \\
\hline 4 & 55 & 4 & 109.42 & 109.8 \\
\hline 4 & 55 & 5 & 8 & 8 \\
\hline
\end{tabular}

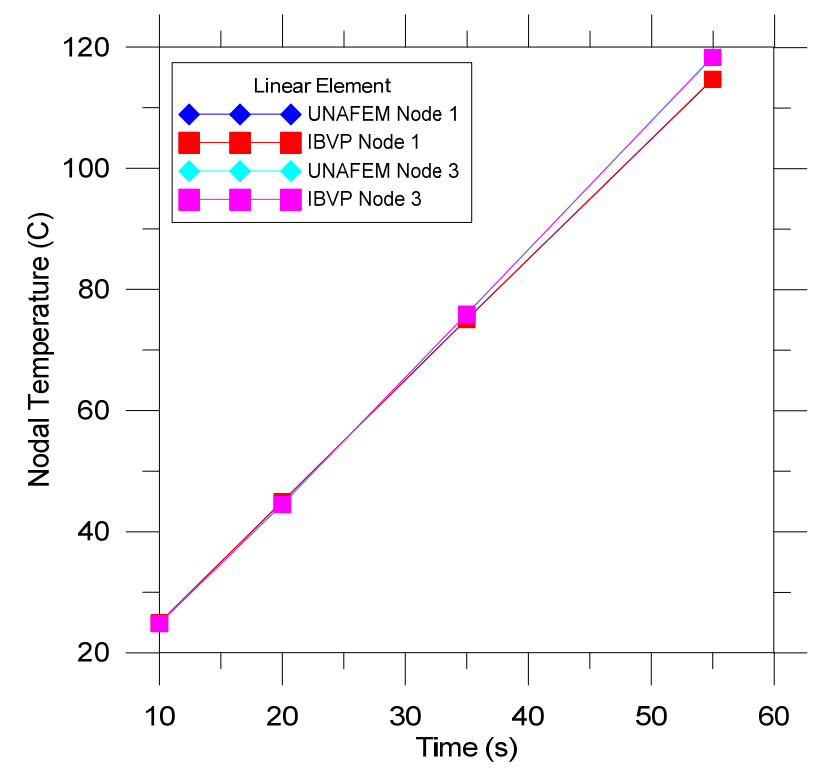

Figure 4-2: Results for select nodal temperatures using linear elements 


\subsubsection{Quadratic Element}

This test uses two quadratic elements with different conductivity values for each element. The left end is insulated and the right end is kept at a temperature of $10^{\circ} \mathrm{C}$ for 20 seconds and then changes to $8^{\circ} \mathrm{C}$ for the remaining time. Again varying number of time steps were used in each time interval, with changing $\Delta \mathrm{t}$ values and $\theta$-values for each. The figure below shows the geometry and parameters for the problem.

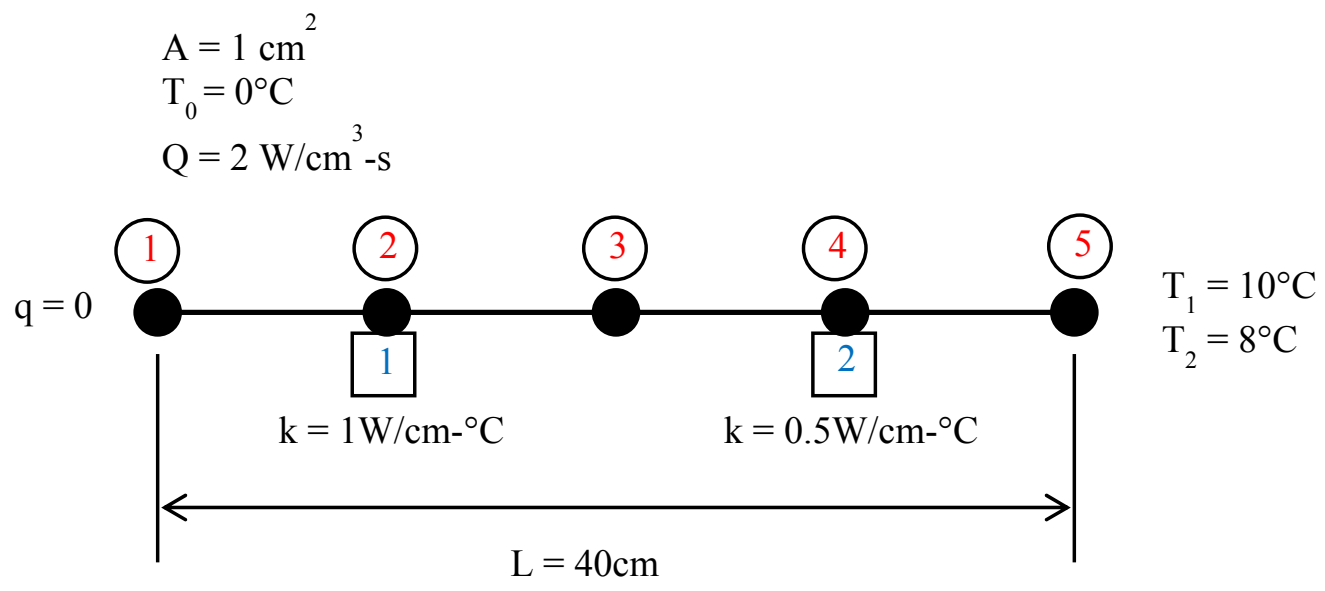

Figure 4-3: Quadratic element

The following tables show the time step data followed by the results for the quadratic element test. The figure represents the nodal temperatures from both programs for node 1 and node 3 . The results match almost exactly from both programs.

Table 4-3: Time Step Data for Quadratic Element

\begin{tabular}{|c|c|c|c|c|c|}
\hline Interval & $\begin{array}{c}\text { Number } \\
\text { of Time } \\
\text { Steps }\end{array}$ & $\boldsymbol{\Delta t} \mathbf{( s )}$ & $\boldsymbol{\theta}$-Value & $\begin{array}{c}\text { Left End } \\
\text { BC }\end{array}$ & $\begin{array}{c}\text { Right } \\
\text { End BC }\end{array}$ \\
\hline 1 & 1 & 10 & 0.67 & NBC 0 & EBC 10 \\
\hline 2 & 2 & 5 & 0.4 & NBC 0 & EBC 10 \\
\hline 3 & 1 & 3 & 0.67 & NBC 0 & EBC 8 \\
\hline 4 & 2 & 10 & 0.33 & NBC 0 & EBC 8 \\
\hline
\end{tabular}


Table 4-4: Results for Quadratic Element

\begin{tabular}{|c|c|c|c|c|}
\hline Time Interval & Time (s) & Node & $\begin{array}{c}\text { IBVP Nodal } \\
\text { Temperature } \\
\left({ }^{\circ} \mathbf{C}\right)\end{array}$ & $\begin{array}{c}\text { UNAFEM Nodal } \\
\text { Temperature } \\
\left({ }^{\circ} \mathbf{C}\right)\end{array}$ \\
\hline 1 & 10 & 1 & 13.16 & 13.16 \\
\hline 1 & 10 & 2 & 13.45 & 13.46 \\
\hline 1 & 10 & 3 & 11.96 & 11.96 \\
\hline 1 & 10 & 4 & 14.53 & 14.54 \\
\hline 1 & 10 & 5 & 10 & 10 \\
\hline 2 & 20 & 1 & 32.66 & 32.66 \\
\hline 2 & 20 & 2 & 33.48 & 33.49 \\
\hline 2 & 20 & 3 & 27.77 & 27.78 \\
\hline 2 & 20 & 4 & 38.43 & 38.43 \\
\hline 2 & 20 & 5 & 10 & 10 \\
\hline 3 & 23 & 1 & 63.13 & 63.10 \\
\hline 3 & 23 & 2 & 62.43 & 62.43 \\
\hline 3 & 23 & 3 & 54.47 & 54.04 \\
\hline 3 & 23 & 4 & 71.42 & 71.92 \\
\hline 3 & 23 & 5 & 8 & 8 \\
\hline 4 & 43 & 1 & 103.5 & 103.6 \\
\hline 4 & 43 & 2 & 101.1 & 101.0 \\
\hline 4 & 43 & 3 & 97.13 & 97.02 \\
\hline 4 & 43 & 4 & 106.2 & 106.6 \\
\hline 4 & 43 & 5 & 8 & 8 \\
\hline
\end{tabular}

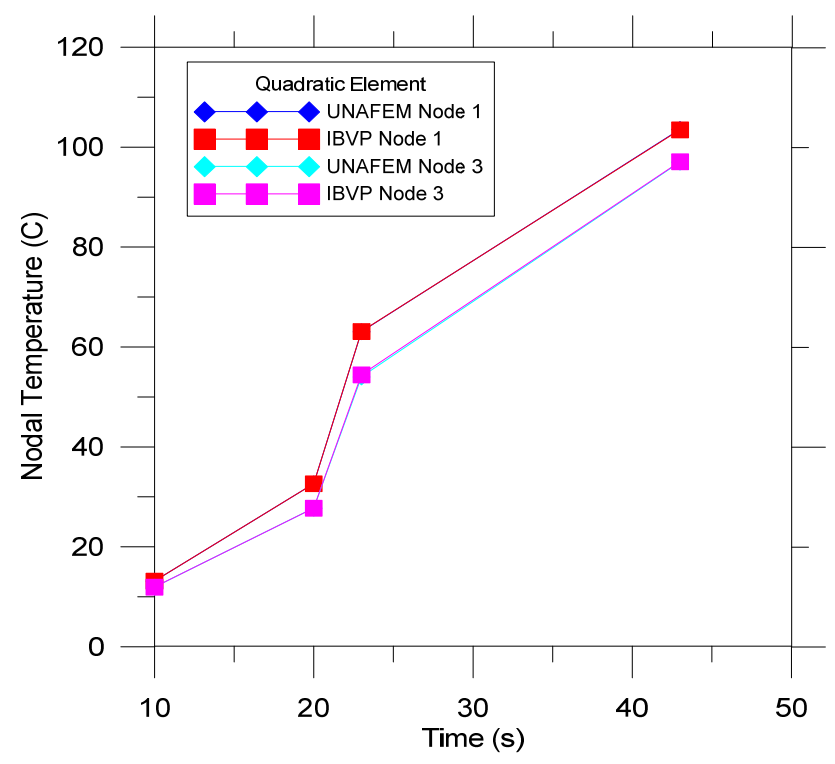

Figure 4-4: Results for select nodal temperatures using quadratic elements 


\subsubsection{Cubic Element}

This test used one cubic element. The left end is insulated and the right end is kept at a temperature of $0^{\circ} \mathrm{C}$ for the entire time. For this problem the $\Delta \mathrm{t}$ and $\theta$-value was kept constant.

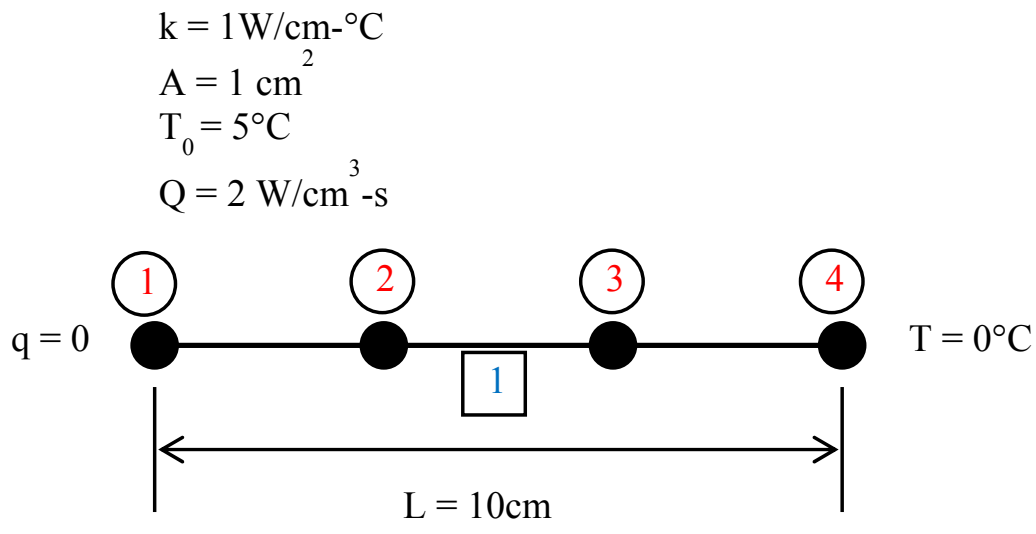

Figure 4-5: Cubic element

The following tables show the time step data followed by the results for the cubic element test in both a table and figure. The results, once again, from both programs are consistent.

Table 4-5: Time Step Data for Cubic Element

\begin{tabular}{|c|c|c|c|c|c|}
\hline Interval & $\begin{array}{c}\text { Number of } \\
\text { Time Steps }\end{array}$ & $\boldsymbol{\Delta t} \mathbf{( s )}$ & $\begin{array}{c}\boldsymbol{\theta}- \\
\text { Value }\end{array}$ & $\begin{array}{c}\text { Left End } \\
\text { BC }\end{array}$ & $\begin{array}{c}\text { Right } \\
\text { End BC }\end{array}$ \\
\hline 1 & 1 & 10 & 0 & NBC 0 & EBC 0 \\
\hline 2 & 1 & 10 & 0 & NBC 0 & EBC 0 \\
\hline 3 & 1 & 10 & 0 & NBC 0 & EBC 0 \\
\hline 4 & 1 & 10 & 0 & NBC 0 & EBC 0 \\
\hline
\end{tabular}


Table 4-6: Results for Cubic Element

\begin{tabular}{|c|c|c|c|c|}
\hline Time Interval & Time (s) & Node & $\begin{array}{c}\text { IBVP Nodal } \\
\text { Temperature } \\
\left({ }^{\circ} \mathbf{C}\right)\end{array}$ & $\begin{array}{c}\text { UNAFEM Nodal } \\
\text { Temperature } \\
\left({ }^{\circ} \mathbf{C}\right)\end{array}$ \\
\hline 2 & 10 & 1 & 20 & 20 \\
\hline 2 & 10 & 2 & 20 & 20 \\
\hline 2 & 10 & 3 & 20 & 20 \\
\hline 2 & 10 & 4 & 0 & 0 \\
\hline 3 & 20 & 1 & 36.07 & 36.15 \\
\hline 3 & 20 & 2 & 46.63 & 46.58 \\
\hline 3 & 20 & 3 & 21.93 & 21.96 \\
\hline 3 & 20 & 4 & 0 & 0 \\
\hline 4 & 30 & 1 & 105.26 & 104.6 \\
\hline 4 & 30 & 2 & 6.94 & 7.53 \\
\hline 4 & 30 & 3 & 61.98 & 61.67 \\
\hline 4 & 30 & 4 & 0 & 0 \\
\hline
\end{tabular}

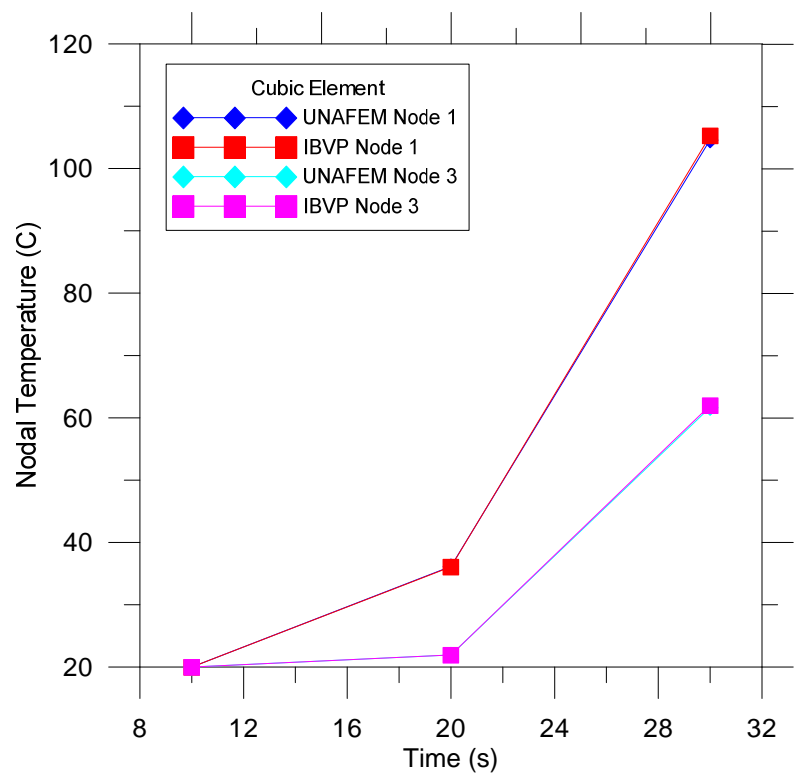

Figure 4-6: Results for select nodal temperatures using cubic elements These were very simplified examples for each element type, but many more test cases were run to ensure that the IBVP program was working correctly. There was only minimal error between the two programs, less than $1 \%$, so the IBVP program was validated for linear transient analysis. Once this portion of the program was verified, the nonlinearity pieces had to be checked. 


\subsection{IBVP nonlinear transient analysis}

The nonlinear portion of the IBVP program solves when conductivity is dependent on temperature and when phase change is involved. For both of these methods ABAQUS v. 6.10 finite element software was used to verify the results. ABAQUS is a very powerful software, but uses the backward difference method to compute transient analysis [33]. As a result, all the tests run through the IBVP program use a value of $\theta=1$ for this comparison. The UNAFEM program also did not have the capability to model mixed boundary conditions, which are important for the later wall design studies, so these conditions are also modeled and verified against ABAQUS.

\subsubsection{Nonlinear conductivity}

When conductivity is dependent on temperature, it was entered into both the IBVP program and ABAQUS as a table of temperature and conductivity values and then linearly interpolated between the values depending on what the current element temperature was. The IBVP program used Newton's method in order to solve the nonlinear problem. ABAQUS uses a combination of a modified Newton's method and secant method for nonlinear problems. As a result, for the initial time steps the values from both programs differ slightly; however, eventually both converge to the same nodal temperatures. A mesh convergence was also done to see if this causes quicker convergence.

The first test uses six linear elements with elements 1-3 that have a temperature dependent conductivity and elements 4-6 that have a constant conductivity. The left end is kept at a constant temperature of $10^{\circ} \mathrm{C}$ and the right end has a mixed boundary condition applied to it with an ambient temperature of $2.33^{\circ} \mathrm{C}$ and convective coefficient 
of $3 \mathrm{~W} / \mathrm{m}^{2}-{ }^{\circ} \mathrm{C}$. A constant $\Delta \mathrm{t}$ value of $10 \mathrm{~s}$ was used. The figure below shows the geometry and parameters for the problem.

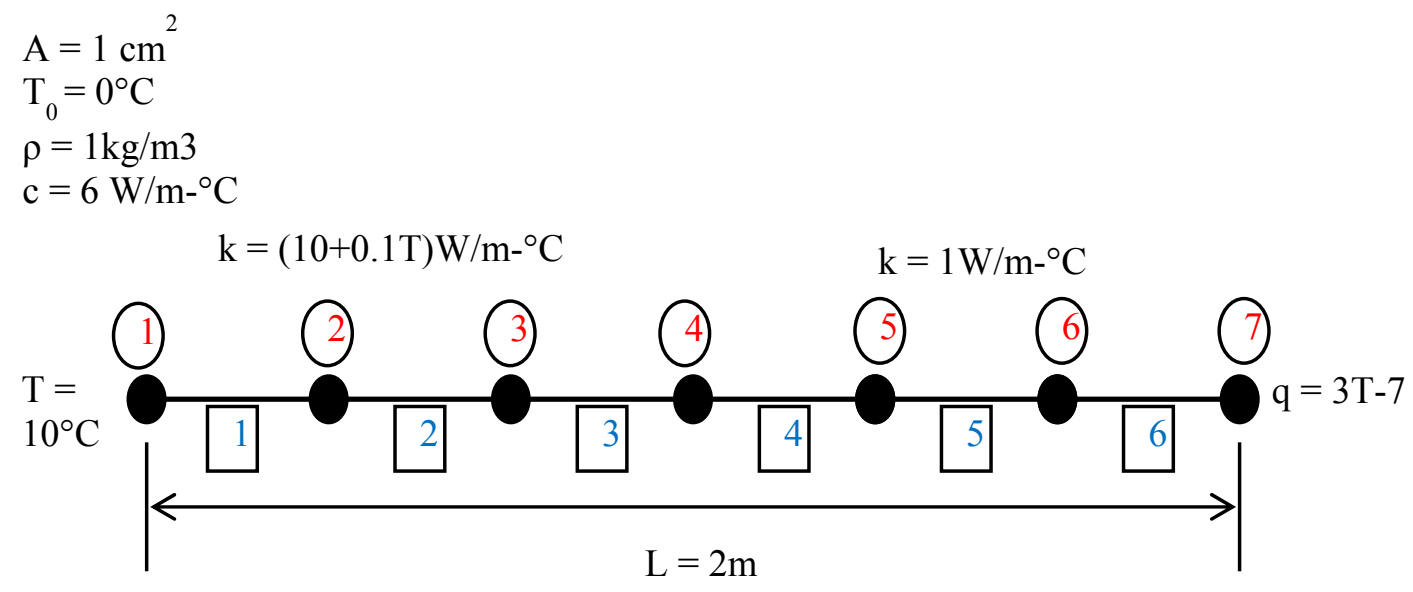

Figure 4-7: Nonlinear Conductivity

The table and figure below display the results for the analysis from both the IBVP program and ABAQUS. Only the nodal temperatures from node 1, 4 and 7 are given. As the table shows, the values initially do not match between the two programs, but eventually converge to the same values quickly. This is due to the difference in the nonlinear solvers that both programs use. 
Table 4-7: Results for six element nonlinear analysis

\begin{tabular}{|c|c|c|c|}
\hline Time (s) & Node & $\begin{array}{c}\text { IBVP Nodal } \\
\text { Temperatures } \\
\left({ }^{\circ} \mathbf{C}\right)\end{array}$ & $\begin{array}{c}\text { ABAQUS Nodal } \\
\text { Temperatures } \\
\left({ }^{\circ} \mathbf{C}\right)\end{array}$ \\
\hline 10 & 1 & 10 & 10 \\
\hline 10 & 4 & 9.478 & 9.054 \\
\hline 10 & 7 & 4.069 & 3.64 \\
\hline 20 & 1 & 10 & 10 \\
\hline 20 & 4 & 9.51 & 9.48 \\
\hline 20 & 7 & 4.127 & 4.071 \\
\hline 30 & 1 & 10 & 10 \\
\hline 30 & 4 & 9.51 & 9.507 \\
\hline 30 & 7 & 4.127 & 4.121 \\
\hline 40 & 1 & 10 & 10 \\
\hline 40 & 4 & 9.51 & 9.509 \\
\hline 40 & 7 & 4.127 & 4.127 \\
\hline 50 & 1 & 10 & 10 \\
\hline 50 & 4 & 9.51 & 9.51 \\
\hline 50 & 7 & 4.127 & 4.127 \\
\hline 60 & 1 & 10 & 10 \\
\hline 60 & 4 & 9.51 & 9.51 \\
\hline 60 & 7 & 4.127 & 4.127 \\
\hline
\end{tabular}

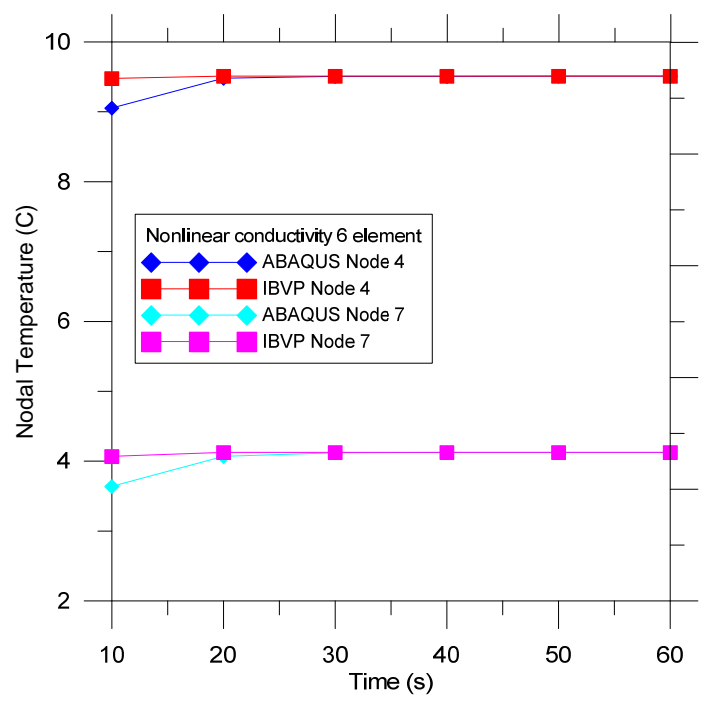

Figure 4-8: Results for nonlinear conductivity using six elements

With only six elements, the results converged quickly but there were slight differences in the temperatures initially. A convergence study shows that even with the use of 200 
elements, the results are still similar. This is because for initial time steps of a nonlinear analysis, ABAQUS uses a different solving technique; however, the results eventually converge to the same values. There is only a $4 \%$ difference in results though, showing IBVP provides fairly similar answers to ABAQUS. This test also validates the use of mixed boundary conditions since that was the type of boundary condition applied to the right end.

Table 4-8: Results for two hundred element nonlinear analysis

\begin{tabular}{|c|c|c|c|}
\hline Time (s) & Node & $\begin{array}{c}\text { IBVP Nodal } \\
\text { Temperatures } \\
\left({ }^{\circ} \mathbf{C}\right)\end{array}$ & $\begin{array}{c}\text { ABAQUS Nodal } \\
\text { Temperatures } \\
\left({ }^{\circ} \mathbf{C}\right)\end{array}$ \\
\hline 10 & 1 & 10 & 10 \\
\hline 10 & 101 & 9.477 & 9.058 \\
\hline 10 & 201 & 4.068 & 3.628 \\
\hline 20 & 1 & 10 & 10 \\
\hline 20 & 101 & 9.509 & 9.479 \\
\hline 20 & 201 & 4.127 & 4.068 \\
\hline 30 & 1 & 10 & 10 \\
\hline 30 & 101 & 9.509 & 9.507 \\
\hline 30 & 201 & 4.127 & 4.121 \\
\hline 40 & 1 & 10 & 10 \\
\hline 40 & 101 & 9.51 & 9.509 \\
\hline 40 & 201 & 4.127 & 4.127 \\
\hline 50 & 1 & 10 & 10 \\
\hline 50 & 101 & 9.51 & 9.51 \\
\hline 50 & 201 & 4.127 & 4.127 \\
\hline 60 & 1 & 10 & 10 \\
\hline 60 & 101 & 9.51 & 9.51 \\
\hline 60 & 201 & 4.127 & 4.127 \\
\hline
\end{tabular}




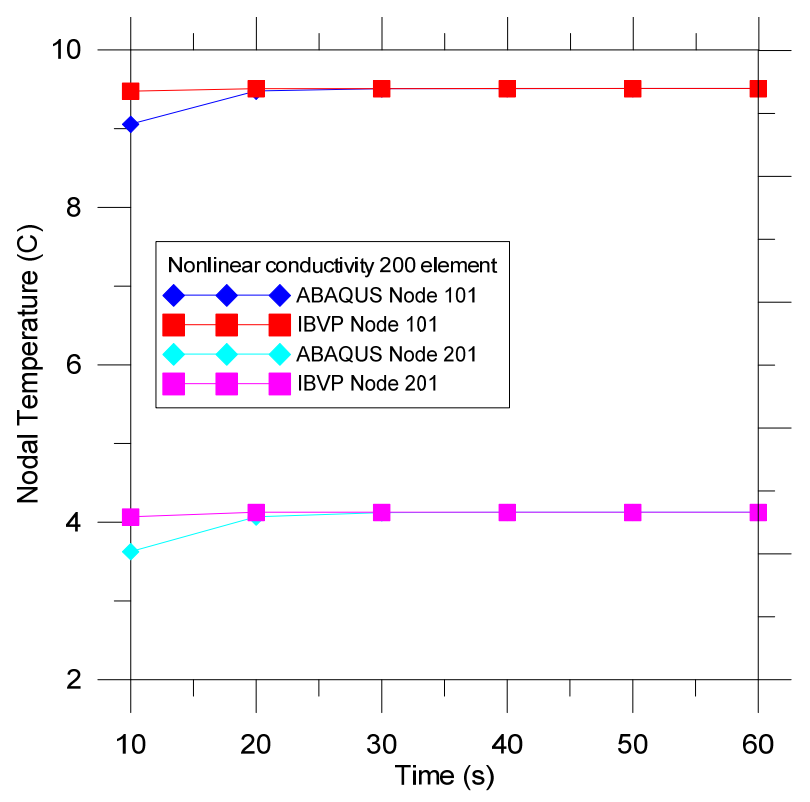

Figure 4-9: Results for nonlinear conductivity using 200 elements

\subsubsection{Latent Heat}

The addition of phase change materials also causes a nonlinear analysis because there is latent heat capacity added only during a certain temperature range. ABAQUS linearly interprets the amount of energy to add in during phase change and this same process was implemented in the IBVP program as well. The results show that using fewer elements is not as accurate and a mesh convergence study was completed in order to validate the IBVP program. Initially very simplified single layer models were run, and then more complex wall models were used to ensure accuracy when running a wall design in IBVP. The first model performed was a single layer of a phase change material with 8 linear elements, constant conductivity and a heat flow of $1 \mathrm{~W} / \mathrm{m}^{2}-{ }^{\circ} \mathrm{C}$ into the left end. The latent heat capacity is significantly larger than the sensible heat capacity and the phase change range is between $4-6^{\circ} \mathrm{C}$. This problem was run for 50 time steps with a $\Delta \mathrm{t}$ of $1 \mathrm{~s}$. 


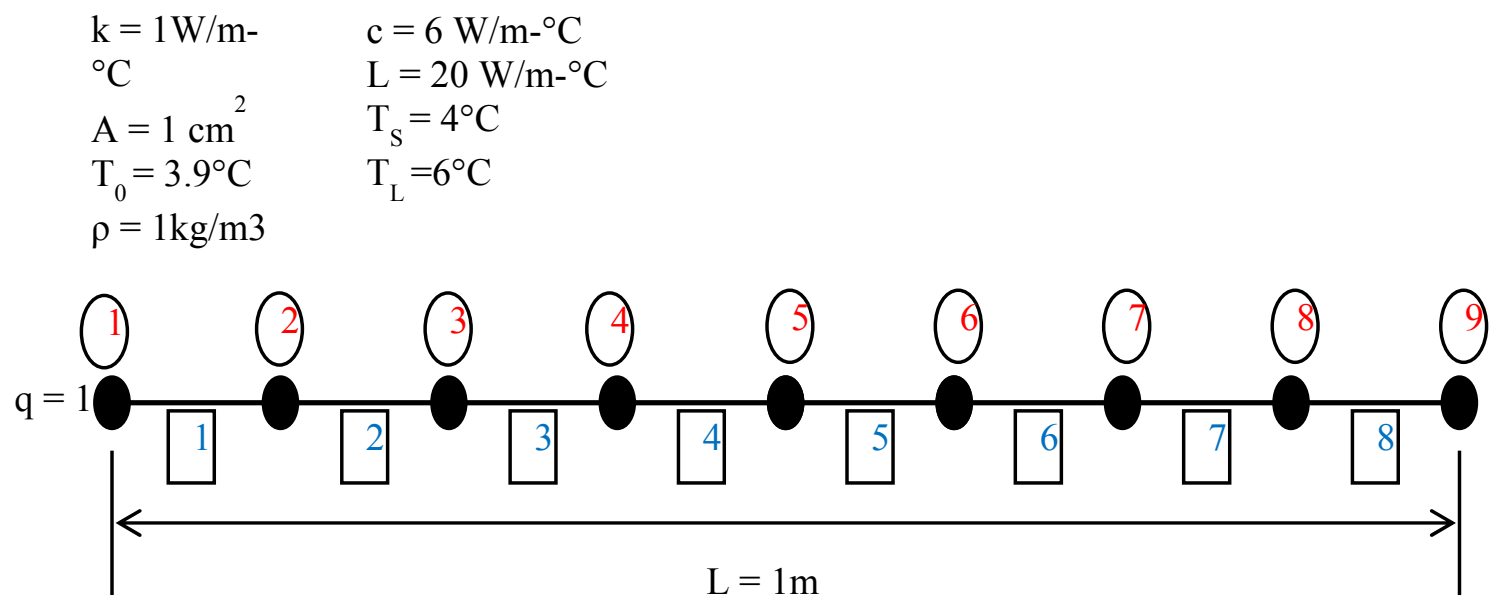

Figure 4-10: Nonlinear latent heat model

The results from this model are shown in the table and figure below. In the table only the temperatures of the two end nodes are shown at only a select few of the time steps. The time steps of major interest are when the material is changing in to and out of phase change. These are the two instances shown in the results and as shown, the IBVP program does not match exactly the results from ABAQUS. 
Table 4-9: Results for 8 element nonlinear analysis

\begin{tabular}{|c|c|c|c|}
\hline Time (s) & Node & $\begin{array}{c}\text { IBVP Nodal } \\
\text { Temperatures } \\
\left({ }^{\circ} \mathbf{C}\right)\end{array}$ & $\begin{array}{c}\text { ABAQUS Nodal } \\
\text { Temperatures } \\
\left({ }^{\circ} \mathbf{C}\right)\end{array}$ \\
\hline 1 & 1 & 4.181 & 4.202 \\
\hline 1 & 9 & 3.946 & 3.946 \\
\hline 2 & 1 & 4.315 & 4.331 \\
\hline 2 & 9 & 3.994 & 4 \\
\hline 3 & 1 & 4.415 & 4.429 \\
\hline 3 & 9 & 4.023 & 4.033 \\
\hline 4 & 1 & 4.499 & 4.512 \\
\hline 4 & 9 & 4.065 & 4.077 \\
\hline 5 & 1 & 4.574 & 4.587 \\
\hline 5 & 9 & 4.115 & 4.127 \\
\hline 25 & 1 & 5.845 & 5.857 \\
\hline 25 & 9 & 5.345 & 5.357 \\
\hline 26 & 1 & 5.907 & 5.92 \\
\hline 26 & 9 & 5.407 & 5.42 \\
\hline 27 & 1 & 5.97 & 5.982 \\
\hline 27 & 9 & 5.47 & 5.482 \\
\hline 28 & 1 & 6.032 & 6.053 \\
\hline 28 & 9 & 5.532 & 5.545 \\
\hline 29 & 1 & 6.095 & 6.122 \\
\hline 29 & 9 & 5.595 & 5.608 \\
\hline 30 & 1 & 6.157 & 6.207 \\
\hline 30 & 9 & 5.657 & 5.673 \\
\hline
\end{tabular}

The following graph shows the temperature values at all time steps for both end nodes.

As seen in the graph and the table the values are much closer for the beginning time steps and then not as exact for the later time steps. 


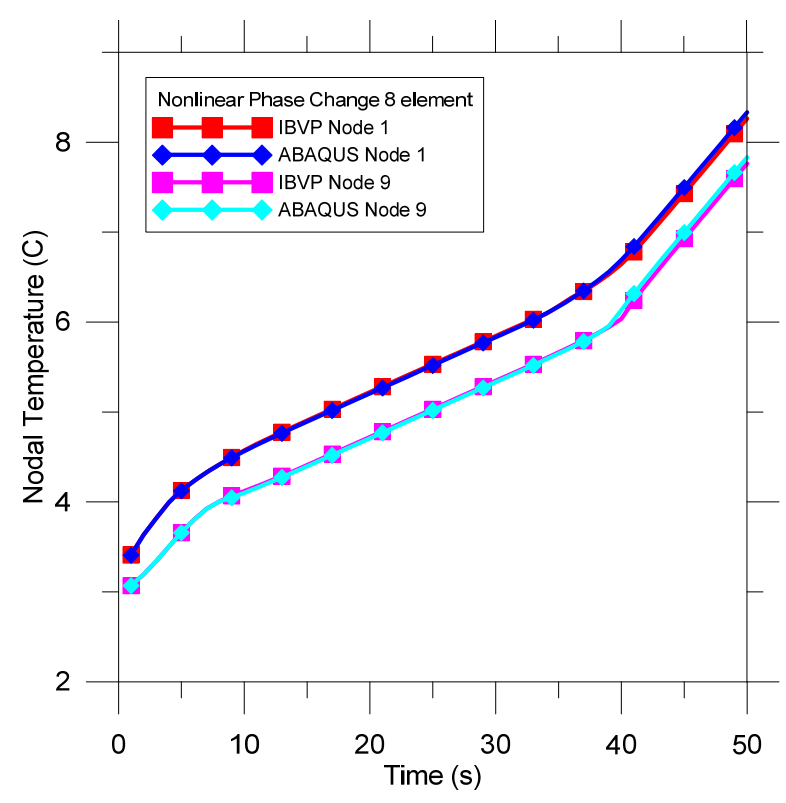

Figure 4-11: Results for latent heat analysis with 8 elements

A mesh convergence was done on this example by increasing the number of elements.

The result from the final convergence model using 100 elements is shown below.

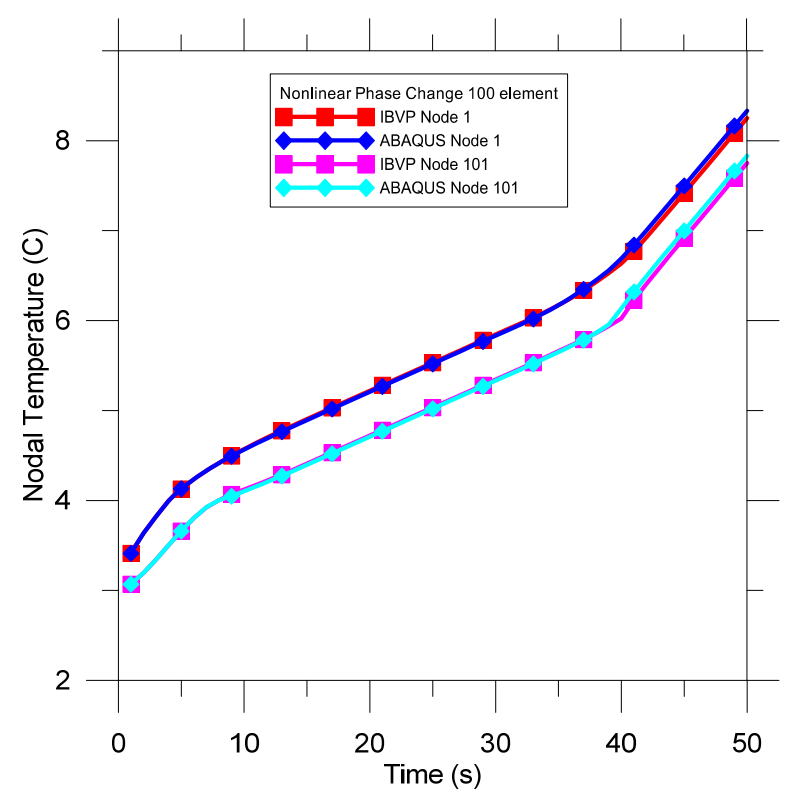

Figure 4-12: Results for latent heat analysis with 100 elements

This figure shows very similar results to the 8 element model. This is due to the computing accuracy used differs between the IBVP program and ABAQUS, which is the reason the results begin to diverge from each other slightly as the time steps increase. 
However, the difference between the two programs values is never more than $1 \%$, so the results from the IBVP program are considered acceptable.

Once these preliminary tests were done, simple studies of two different wall designs were looked at. For these models, both the nodal temperatures and the amount of energy through the wall were looked at to ensure that the IBVP program was working correctly and produced similar answers as ABAQUS. The two wall models were ran for 48 hours with a $\Delta t$ of 1 hour and mixed boundary conditions applied to both ends of the wall. The first wall model is a three layer model with concrete on the exterior and a phase change material in the center. There were 40 elements that made up the model. The mixed boundary conditions varied over time (the specific values for the boundary conditions are described later in this paper).

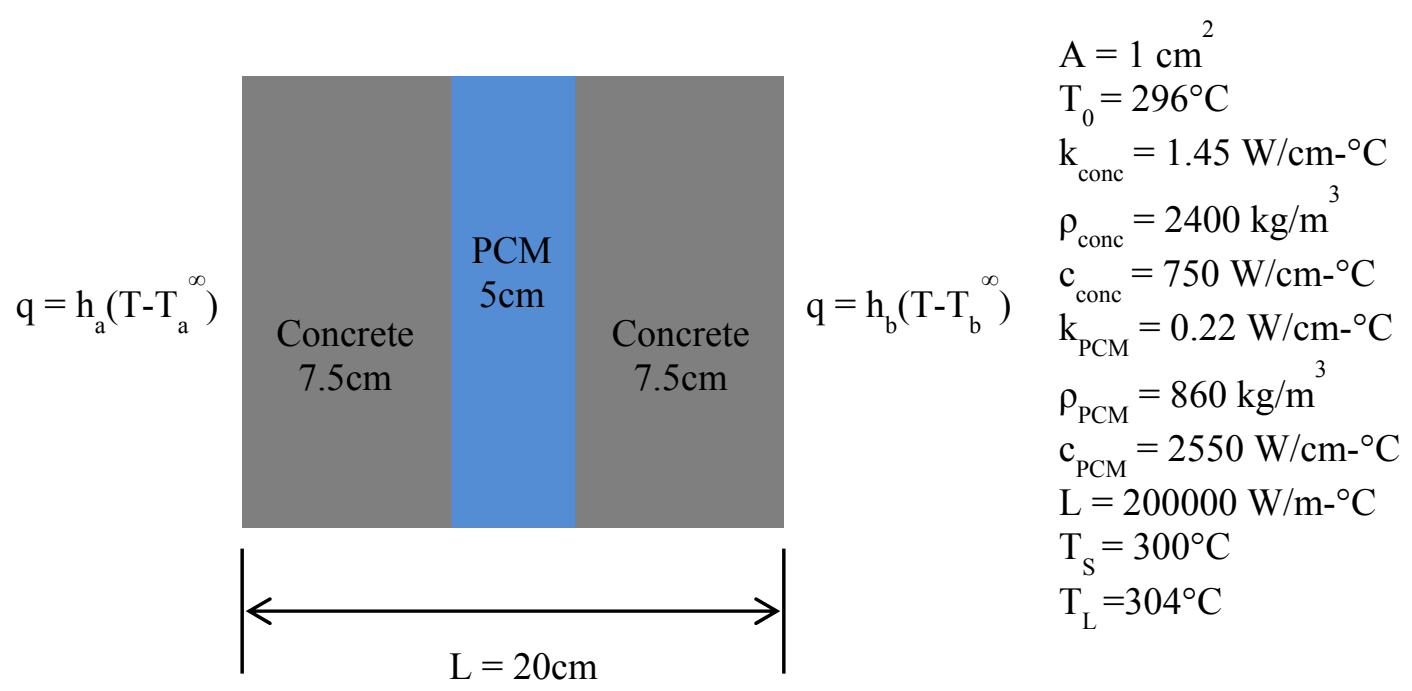

Figure 4-13: Three layer wall model

The results for the nodal temperatures and the energy flow through the wall are shown below. The nodal temperatures shown are for the two end nodes and the energy flow is calculated as the energy through the right end of the model. 


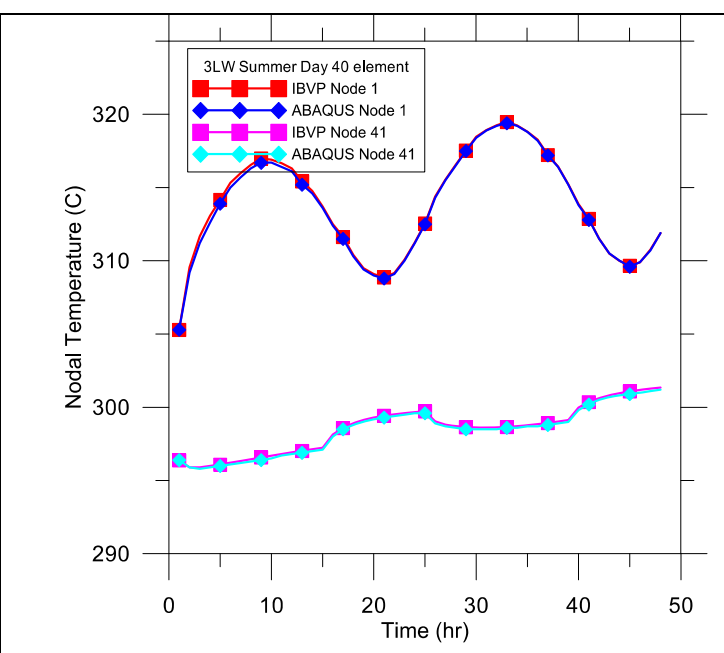

(a)

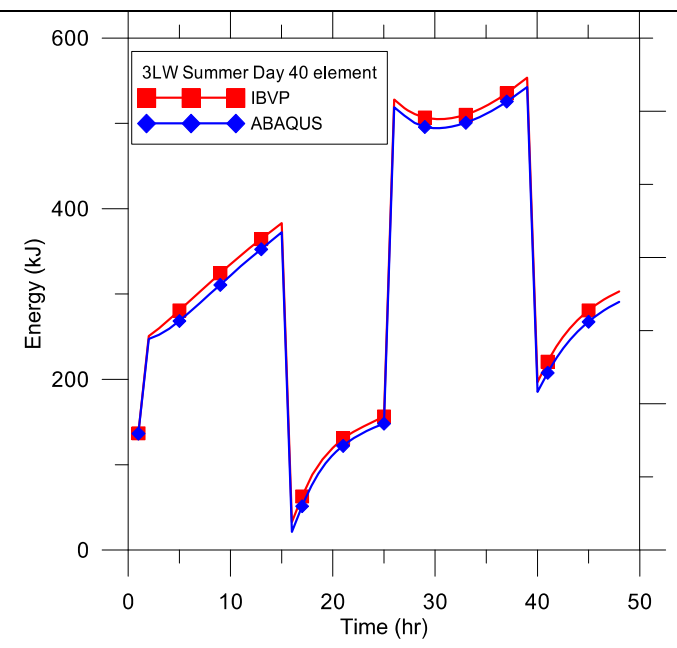

(b)

Figure 4-14: (a) Nodal temperature results for the three layer wall model, (b) energy flow results for the three layer wall model

As the figure show the nodal temperatures from both the IBVP program and ABAQUS matched for the entire 48 hours. The energy flow graph shows slight differences between the values over the time period. This is because energy flow is computed using the element heat flux values which are derived from the nodal temperatures. As stated earlier, ABAQUS and the IBVP program use different precision when computing both the nodal temperatures and the heat flux values, so once all the factors are multiplied in to compute energy flow there becomes very slight differences of about $1 \%$ for the total energy flow over the entire 48 hour period.

The second wall model was a five layer model with two layers of phase change material and three layers of concrete. The same overall thickness was used and the same boundary conditions were applied to each side of the model. The materials were given the same material properties to ensure that the IBVP program works for several layers of phase change materials. 


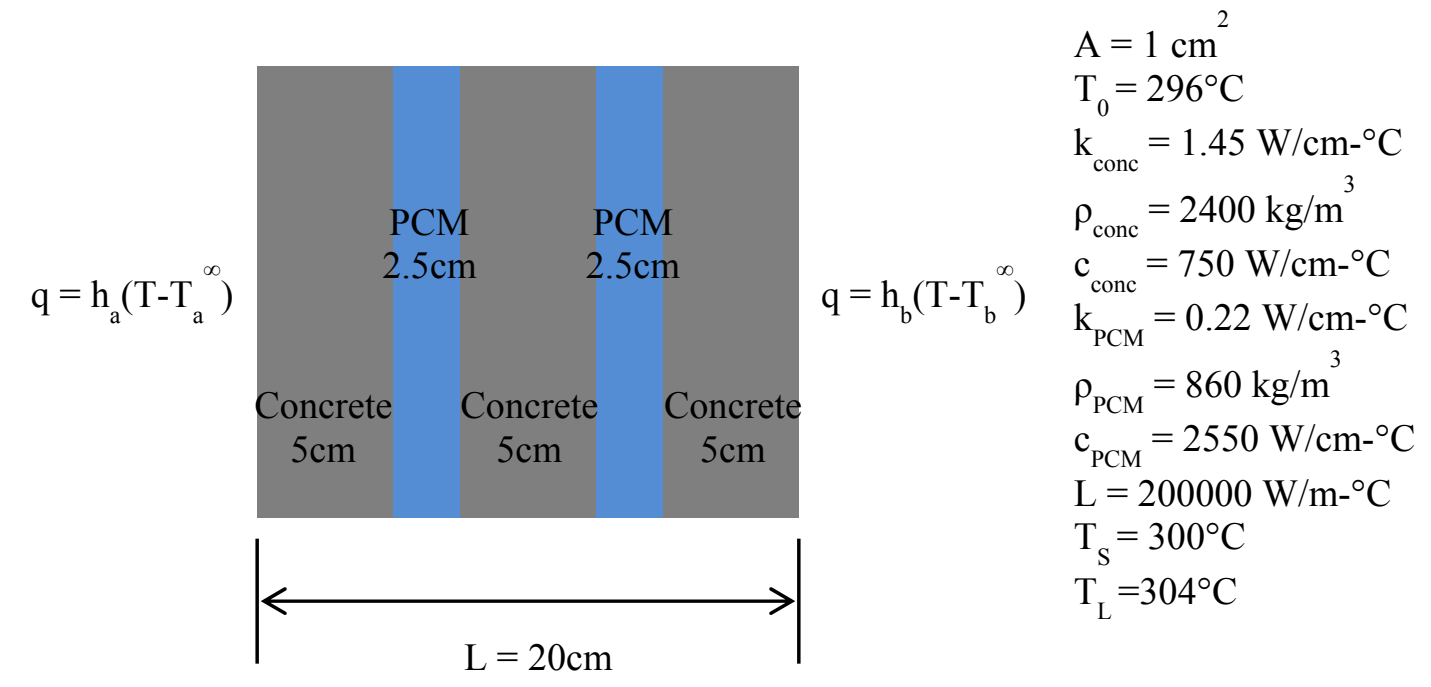

Figure 4-15: Five layer wall model

The results for the nodal temperatures and the energy flow through the five layer wall are shown below. The nodal temperatures are for the two end nodes and the energy flow is calculated as the energy through the right end of the model.

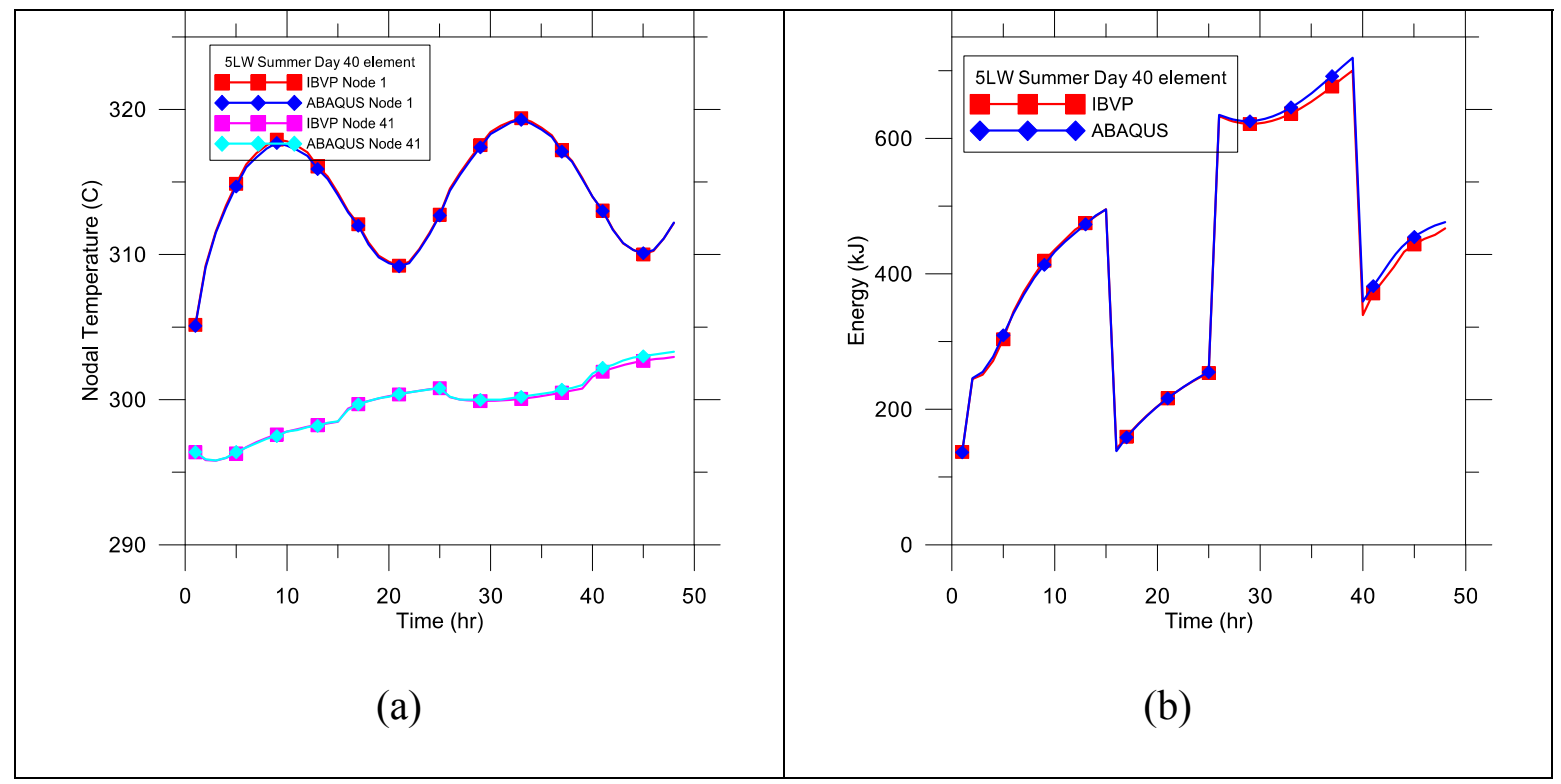

Figure 4-16: (a) Results for nodal temperatures of the five layer wall model, (b) Results for the energy flow through the five layer wall model 
The results for the five layer wall model show the same trend as the three layer wall model. These two examples verified that IBVP works for many different layers of materials with many different material properties.

As a result of all these different trial problems and many others, the IBVP program was validated to be used as a finite element analysis program. The results for both the nodal temperatures and the element fluxes that are computed in the program are shown to be accurate as compared to ABAQUS. This program was then integrated into the WallDesign program which contains many other important functions to post process these results to create the fully functional wall design package.

\subsection{WallDesign Program}

The WallDesign program initially started as a program which used ABAQUS for the FEA; however, now both ABAQUS and IBVP are incorporated into the program and either can be used. The WallDesign program has the capability of reading an input file to create the FEA model and then uses the results for the temperatures and fluxes from the finite element analysis to perform its other functions of post processing these results and optimizing the wall design.

\subsubsection{WallDesign Post Processing}

The post processing of the FEA results are important in order to calculate two parameters, the energy flow through the wall (this will be referred to hence forth as energy flow through the inner face: EFTIF) and the efficiency of the PCM layer(s) (PCME). The EFTIF is a measure that is correlated to the amount of energy an HVAC system would use. The PCME is a measure of how much of the PCM has stored or released energy compared to the total storage capacity of each PCM layer. 
Energy flow through the inside face means the energy flow can take place from the outside of the wall to the inside or from the inside to the outside. However, the total amount of energy flow is summed over the designated time period to represent a comparative amount of energy an HVAC system would be using in a day to keep the interior at the ambient indoor temperature. The more efficient the wall system is the smaller the EFTIF will be and the less energy an HVAC system will use. EFTIF is calculated by using the heat flux values, $\tau_{x}$ (rate of heat energy transfer through a unit area) from the finite element analysis to compute the energy flow through all the elements on the inside face of the wall as shown in the following equation. A unit width is assumed for each wall model. EFTIF is the amount of heat flux across an area of a wall in a given amount of time.

$$
\text { EFTIF }=\int_{t_{i}}^{t_{f}} \int_{0}^{1} \tau_{x} d y d t
$$

The PCME value computes the amount of PCM that is actually being used for energy storage during a certain time period. The total energy storage capacity of any PCM layer is a function of the PCM mass, the sensible and latent heat capacity of the PCM and the thickness of the layer of PCM. The amount of sensible or latent energy stored during a certain time step is a function of the change in the temperature over that time step. The comparison of the amount of energy stored during a time step to the total energy storage capacity is the PCME; this is shown in the equation below. The more efficient the PCM layer is, the more cost effective it will be when placed in a wall design. 


$$
P C M E=\frac{\text { Energy stored in PCM }}{\text { Energy storage capacity of PCM }} \times 100
$$

Both the EFTIF and PCME values were verified by hand calculations performed and extensive studies done previously (24).

\subsubsection{WallDesign Optimization}

The original optimization program was developed as a standalone operating program that could optimize any given problem. There were many test cases performed to verify that the optimizers worked correctly. Some of these test cases included the three bar truss problem, the Rosen-Suzuki problem, a welded beam test and a pressure vessel test (25). This optimization program was then integrated as an optimizing toolbox into the WallDesign program and was quickly tested for accuracy when optimizing wall design models.

The optimization part of the WallDesign program provides a quick and efficient method of determining the best design based on the overall goal and constraints. The main goal of this tool is to minimize the objective function, which is typically a cost function. Since the optimizer can manipulate both continuous and discrete variables, each type of design variable was tested together and separately once integrated in the WallDesign program. All tests produced valid results with much better final objective functions than the initial designs, so it was determined that this toolbox was acceptable and a major benefit to the overall program.

\subsection{Overall Program Integration}

The final product is a wall design package that can analyze a design, produce output on the energy data and optimize the design. These three functions can all be used as separate 
programs, but their integration creates a powerful design tool for walls embedded with PCMs. The following figure shows the overall flow of the entire program. 


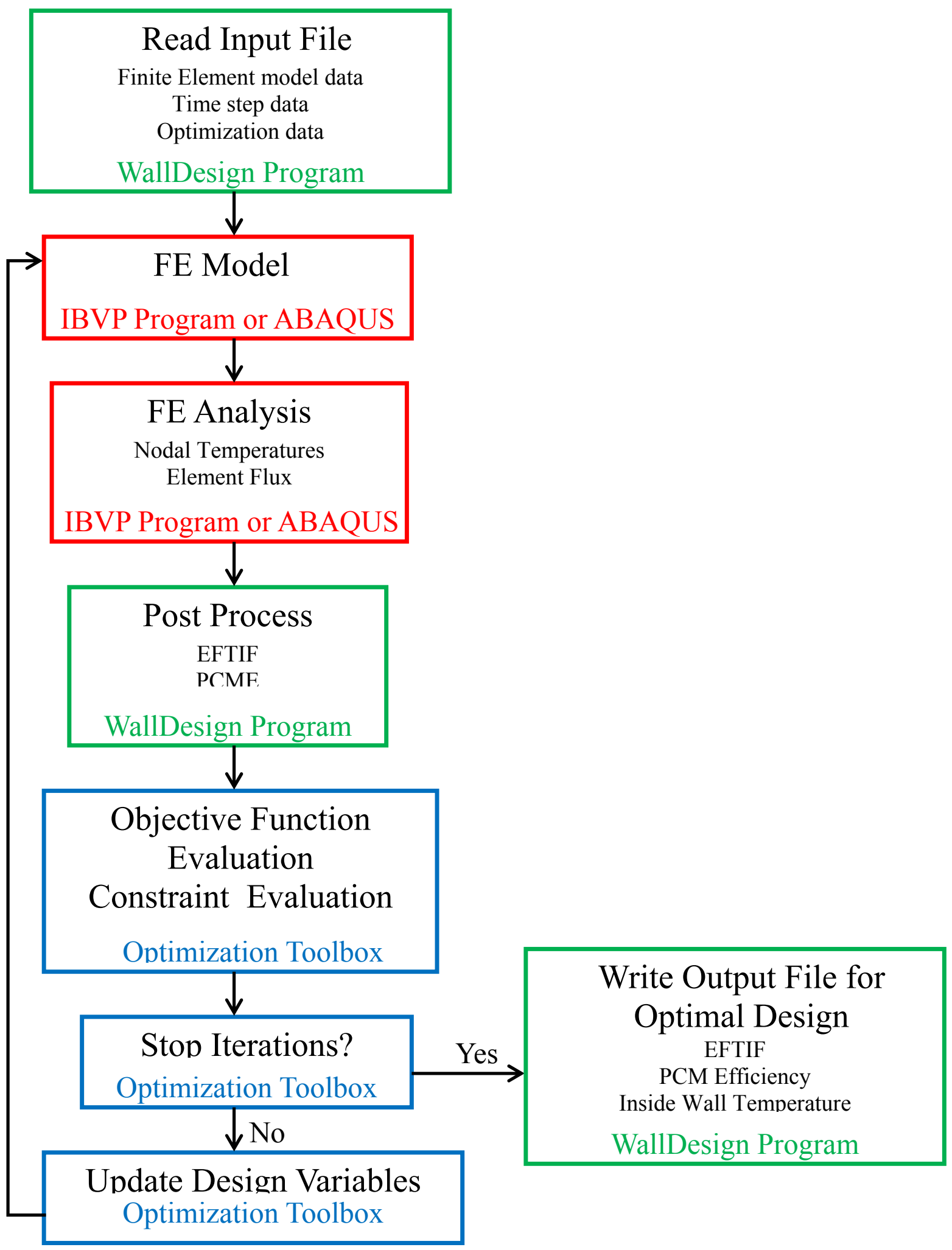

Figure 4-17: Wall Design program 


\section{WALL MODELS AND RESULTS}

The final wall design package can be utilized to solve any sort of layered wall design problem along with optimizing any design. Phase change materials are still a newer technology and as a result are very costly. Placing a large amount of PCM in a wall could potentially cost more than the energy savings this layer would provide. As a result, the balance point between adding PCM and the energy savings must be looked at. The optimizing tool allows for this optimal point to be determined. This chapter will focus on the optimization of two different wall designs and how the program was able to find the lowest overall cost of the wall when considering both material and energy costs. A typical concrete wall will be taken as the building material and the overall goal of this study is to determine the optimal amount of PCM to incorporate in the concrete wall. The implications of the layer thicknesses and PCM material properties on the energy consumption of the wall will be looked at in order to understand how these affect the final design. The objective of the study is to minimize an overall cost value that includes minimizing the energy needed to maintain the interior temperature of a room at a specified human comfort level and the cost of the building materials. Also, the addition of

a PCM layer into a concrete wall reduces the structural integrity of the wall. This reduction in structural capacity will also be looked at to ensure the wall designs would still be structurally acceptable for use as a building element.

\subsection{Temperature Conditions}

This study was performed for the weather conditions of Phoenix, AZ (United States) that has a subtropical desert climate. The average outdoor temperature profile of Phoenix consists of three different temperature conditions over a typical year: summer 
temperature days, winter temperature days and spring/fall temperature days. Data from June $14^{\text {th }}, 2011$ is taken as a typical summer day with temperature range of $37-51{ }^{\circ} \mathrm{C}$, a typical winter day data is taken from January $1^{\text {st }}, 2011$ with a range of $3-14^{\circ} \mathrm{C}$ and the spring/fall day data is from March $4^{\text {th }}, 2011$ and has a temperature range of $16-34^{\circ} \mathrm{C}$ as shown in Figure 5-1.

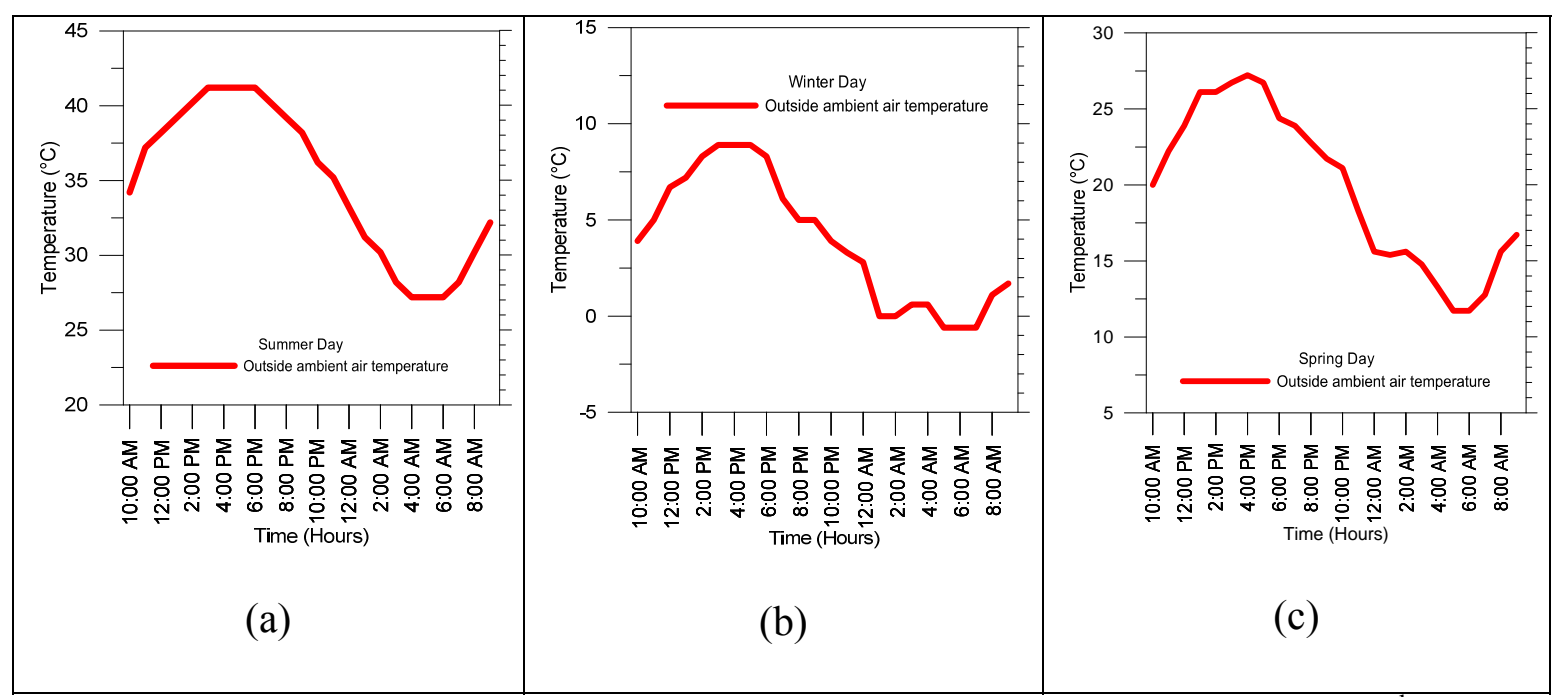

Figure 5-1: Ambient air temperature for Phoenix without radiation (a) on June $14^{\text {th }}, 2011$, (b) on January $1^{\text {st }}, 2011,(c)$ on March $4^{\text {th }}, 2012$.

Instead of applying solar radiation as a flux on the outside face of the wall, an equivalent temperature $\left(T_{\text {eq }}\right)$ is calculated. Radiation effect during sunshine hours is calculated as

$$
T_{e q}=T_{e}+\frac{\alpha S}{h_{e}}
$$

In the above equation, $T_{e}$ is the ambient temperature without the radiation effects $\left(\right.$ in $\left.{ }^{\circ} \mathrm{C}\right)$, $\alpha$ is the absorption coefficient (unit less), $\mathrm{S}$ is the solar insolation (defined as rate of delivery of solar radiation per unit area with units of $\mathrm{kWh} / \mathrm{m}^{2}$ per day), taken as $309.2 \mathrm{~W}$ $\mathrm{s} / \mathrm{m}^{2} / \mathrm{s}$, and $\mathrm{h}_{\mathrm{e}}$ is the convective heat transfer coefficient, assumed to be $20 \mathrm{~W} / \mathrm{m}^{2}-{ }^{\circ} \mathrm{C}$, and $\alpha$ is the solar absorption coefficient taken as $0.65[46,47,48]$. The effective ambient temperature profiles that include the equivalent radiation effects are shown in Figure 5-2. 
In this study, we take the indoor air to be controlled by a heating, ventilation and airconditioning (HVAC) system and is referred to as inside face air temperature (IFAT) in the remainder of the paper. The IFAT values are taken to reflect perceived human comfort conditions for different times of the year and different times of the day - summer and spring/fall days: $20^{\circ} \mathrm{C}$ during the daytime and $25^{\circ} \mathrm{C}$ during the nighttime; winter days: $20^{\circ} \mathrm{C}$ during the daytime and $16^{\circ} \mathrm{C}$ during the nighttime. These values are selected based on the fact that Phoenix has: (a) a drier climate (lower humidity) than most places permitting a higher indoor target temperature with the same comfort level, and (b) that during warm and hot days, evenings are hot and the nighttime cooling takes place gradually.

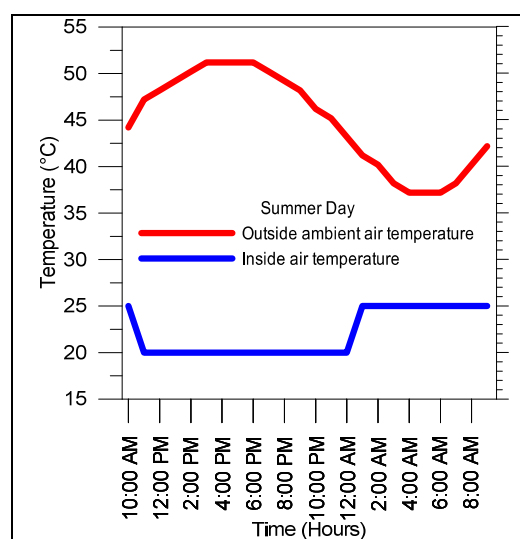

(a)

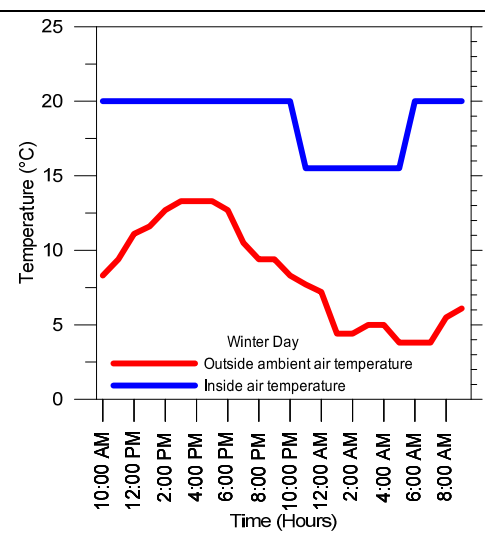

(b)

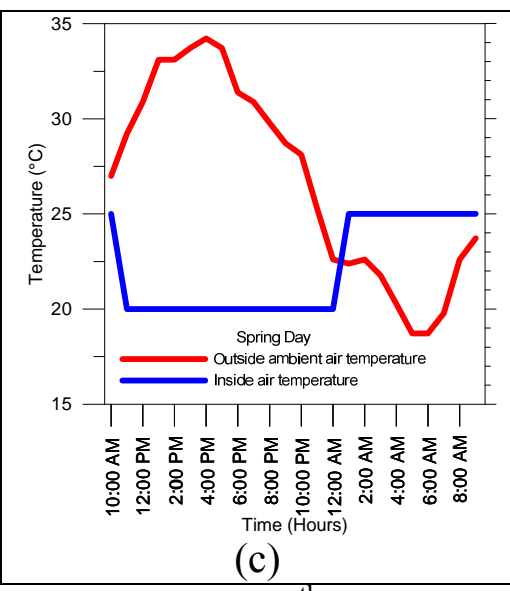

(c)

Figure 5-2: Ambient air temperature for Phoenix with radiation (a) on June $14^{\text {th }}, 2011$, (b) on January $1^{\text {st }}, 2011$, (c) on March $4^{\text {th }}, 2012$

\subsection{Material Properties}

Two types of materials are used in a typical design - conventional portland cement concrete made using normal weight aggregates and PCM. The properties of the materials considered in this study are listed in Table 5-1. 
Table 5-1: Material Properties

\begin{tabular}{|c|c|c|c|c|c|c|}
\hline Name & $\begin{array}{c}\text { Density } \\
\left(\mathbf{k g} / \mathbf{m}^{\mathbf{3}}\right)\end{array}$ & $\begin{array}{c}\text { Conductivity } \\
\left(\mathbf{W} / \mathbf{m}-{ }^{\circ} \mathbf{C}\right)\end{array}$ & $\begin{array}{c}\text { Specific } \\
\text { Heat }(\mathbf{J} / \mathbf{k g}- \\
\left.{ }^{\circ} \mathbf{C}\right)\end{array}$ & $\begin{array}{c}\text { Latent } \\
\text { Heat } \\
(\mathbf{J} / \mathbf{k g})\end{array}$ & $\begin{array}{c}\text { Solidus } \\
\mathbf{T e m p} \\
\left({ }^{\circ} \mathbf{C}\right)\end{array}$ & $\begin{array}{c}\text { Liquidus } \\
\text { Temp } \\
\left({ }^{\circ} \mathbf{C}\right)\end{array}$ \\
\hline $\begin{array}{c}\text { Normal } \\
\text { Concrete }\end{array}$ & 2400 & 1.45 & 750 & - & - & - \\
\hline PCMA & 800 & 0.20 & 2400 & 169000 & 27 & 31 \\
\hline PCMB & 860 & 0.22 & 2100 & 185000 & 23 & 27 \\
\hline PCMC & 850 & 0.22 & 1830 & 189000 & 27 & 31 \\
\hline PCMD & 860 & 0.22 & 2550 & 200000 & 27 & 31 \\
\hline PCME & 300 & 0.25 & 2500 & 110000 & 22 & 25 \\
\hline PCMF & 860 & 0.22 & 2410 & 185000 & 10 & 14 \\
\hline PCMG & 860 & 0.22 & 2000 & 180000 & 6 & 10 \\
\hline
\end{tabular}

Two different types of PCMs are investigated - those with a phase change range that would work on hot days and those for cold days in order to handle the range of temperatures in a climate like Phoenix. Thermal energy storage can be the result of either sensible heat storage or latent heat storage. All materials have an ability to store sensible energy, but phase change materials can also store latent energy. The amount of latent energy that can be stored in a PCM is much larger than its sensible energy storage capacity as seen in Table 5-1. This mechanism of latent energy storage is the same for both hot and cold day PCMs; they store energy when heated up and change to a liquid state. There is very minimal volume change during this phase transition so these materials work well when used in bulk and when encapsulated [2]. When the phase change material is cooled back to its solid state the energy that was stored is released. The following figure represents the large amount of increase in energy stored in PCMs during the phase change range for both a cold day PCM and a hot day PCM. 


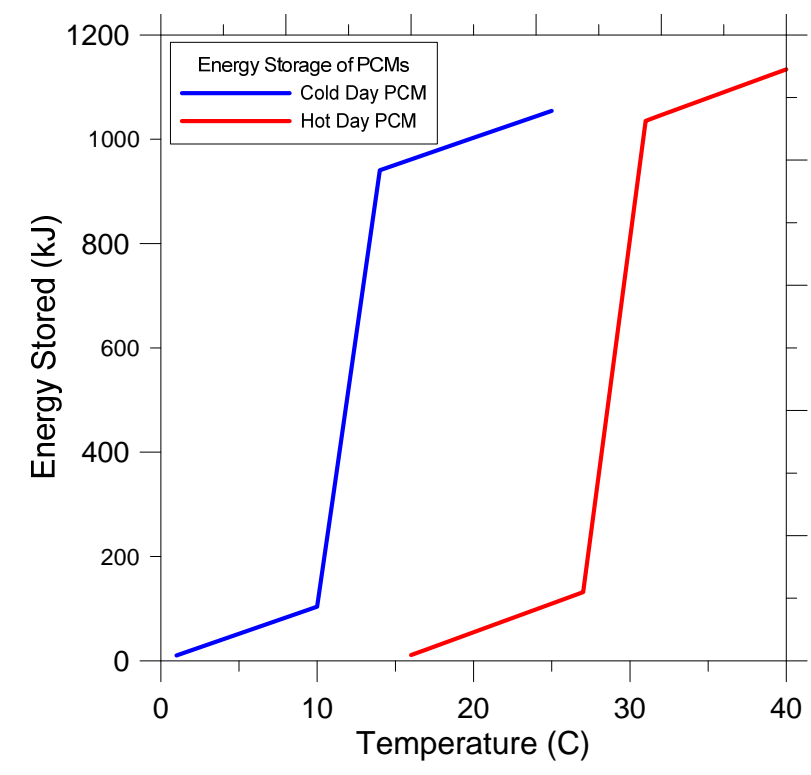

Figure 5-3: Energy storage in hot and cold PCMs

The amount of energy stored can be calculated for each material to understand the differences between the various PCMs in this study, both when the material is in phase change range (latent energy) and when it is not (sensible energy). A layer thickness of $0.005 \mathrm{~m}$ was used with an area of $1 \mathrm{~m}^{2}$ and with $1^{\circ} \mathrm{C}$ of temperature change. These values (see Table 5-2) show which PCMs are candidate materials for hot and cold days.

Table 5-2: Material Energy Storage Capacities (5 mm thick)

\begin{tabular}{|l|c|c|l|l|}
\hline Material & $\begin{array}{c}\text { Solidus } \\
\left.\text { Temp ( }{ }^{\circ} \mathbf{C}\right)\end{array}$ & $\begin{array}{c}\text { Liquidus } \\
\text { Temp }\left({ }^{\circ} \mathbf{C}\right)\end{array}$ & $\begin{array}{c}\text { Latent Energy } \\
\text { Capacity } \\
\left(\frac{J}{{ }^{\circ} C-m^{2}}\right)\end{array}$ & $\begin{array}{c}\text { Sensible } \\
\text { Energy } \\
\text { Capacity } \\
\left(\frac{J}{{ }^{\circ} C-m^{2}}\right)\end{array}$ \\
\hline Concrete & - & - & - & 9000 \\
\hline PCMA & 27 & 31 & 169000 & 9600 \\
\hline PCMB & 23 & 27 & 198875 & 9030 \\
\hline PCMC & 27 & 31 & 200813 & 7778 \\
\hline PCMD & 27 & 31 & 215000 & 10965 \\
\hline PCME & 22 & 25 & 55000 & 3750 \\
\hline PCMF & 10 & 14 & 198875 & 10363 \\
\hline PCMG & 6 & 10 & 193500 & 8600 \\
\hline
\end{tabular}


Five hot-climate and two cold-climate PCMs are looked at as candidate materials. While there are tens of commercially available materials, those PCMs with material properties as well as cost figures were readily available were selected.

\subsection{Wall Geometry and Analysis}

As stated earlier, two wall layouts are considered in this study. The first is a three layer wall $(3 \mathrm{LW})$ model where the inside and the outside layers are normal concrete $(\mathrm{NC})$ and the middle layer is composed of PCM as shown in Figure 5-4(a). The second model is a five layer wall (5LW) model as shown in Figure 5-4(b). For both models, the overall wall thickness is taken as $0.20 \mathrm{~m}$ ( 8 in, which corresponds to typical precast concrete structural wall thickness for industrial and high rise buildings). 


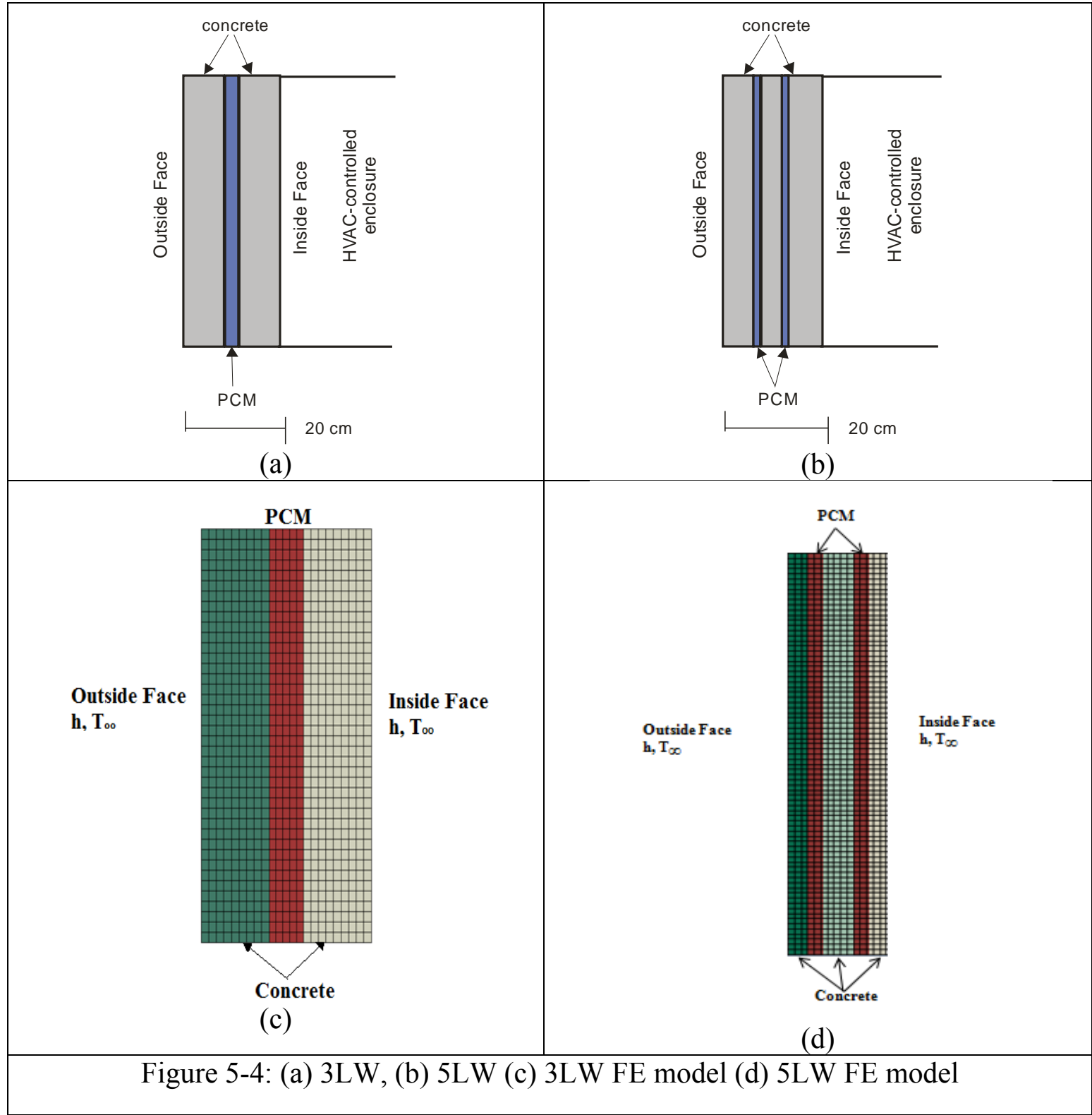

\subsubsection{Finite Element Model for Thermal Analysis}

The wall is modeled for the multi-physics analysis using the finite element method. Both the thermal performance and structural performance are evaluated with each wall configuration. ABAQUS Version 6.10 is used as the finite element program for the stress 
analyses, and ABAQUS and the IBVP program are used for the thermal analysis to verify the results.

For the thermal analysis a one-dimensional heat flow through the wall is assumed. Mixed boundary conditions are imposed on the outside and inside faces. The ambient temperatures used as inputs to the model are obtained from Figure 5-2. The exterior convective heat transfer co-efficient, $h_{e}$ is taken as $20 \mathrm{~W} / \mathrm{m}^{2}-{ }^{\circ} \mathrm{C}$ and the interior convective heat transfer co-efficient, $h_{\text {int }}$ is taken as $5 \mathrm{~W} / \mathrm{m}^{2}-{ }^{\circ} \mathrm{C}$ [35].

In addition it is assumed that (a) all the concrete and PCM layers are homogenous and isotropic, (b) thermal properties of concrete and PCM are constant and temperature independent, (c) convective flow, if any, inside the liquid PCM is ignored, (d) there is no interfacial resistance between the layers, and (e) the top and the bottom surfaces are perfectly insulated (24). The air and the air flow in the HVAC-controlled enclosure are not modeled. The initial temperature of the entire wall is assumed to be the average of the initial ambient temperature of the outside and the inside faces of the wall. A previous convergence study was carried out in ABAQUS to determine the optimal time step values and mesh size to use for the analysis. Models were analyzed for a period of 7 days using time steps of 15 minutes, 30 minutes and 60 minutes with varying element sizes. The results indicate that the system response stabilized after the first 24 hours and generally showed a cyclic pattern, and the final results at the end of the week are consistent [35]. Based on the results of the convergence study, the following model parameters were used in all subsequent ABAQUS finite element analyses: (a) element size of $0.25 \mathrm{~cm}$, (b) 60 minute time step, and (c) duration of analysis as first 48 hours with the reported results for the second 24 hours. As shown in the previous chapter, mesh 
convergence was done for the IBVP program as well, and the following parameters are used in all IBVP finite element analyses; (a) linear elements with a size of $0.25 \mathrm{~cm}$, (b) 60 minute time step, and (c) duration of analysis as first 48 hours with the reported results for the second 24 hours. From these models, the results for the second 24 hour period was used to compute the EFTIF values and PCME values to study the thermal efficiency of each wall design.

\subsubsection{Finite Element Model for Structural Performance}

The structural analysis of this type of wall was also important to consider due to the use of PCM for some layers of the wall and thus making the concrete layers thinner. It was assumed that the wall is not a load bearing wall and is only subjected to wind loads calculated for Phoenix, AZ. The wind speed is taken as $90 \mathrm{mph}$ to create a load of $737 \mathrm{~Pa}$ acting on the outside face of the wall. The concrete material is assumed to have a compressive strength of $27580 \mathrm{kPA}$ with a Poisson's ratio of 0.15 .

Assuming that the PCM has no structural strength, in the finite element model, the layers that are PCM are modeled as void space with a $0.05 \mathrm{~m}$ border of concrete material on the edges to connect one concrete layer to the adjacent one, so the wall is connected together around the edges even though there are void spaces in the center for the PCM layers. The load was applied as a normal pressure to the outside face of the wall. The four corners on the inside face of the wall were assumed to mimic pin supports. A mesh convergence study was carried out to find the optimal mesh configuration using both computational effort and accuracy (predicting principal stresses and maximum displacement) as metrics. The element sizes varied from $0.1 \mathrm{~m}$ to $0.0125 \mathrm{~m}$ using first order tetrahedral elements. Based on the results of the convergence study, the following model parameters were used 
for the structural finite element analyses: (a) element size of $0.0125 \mathrm{~m}$ and (b) 4-noded tetrahedral elements (C3D4). The final FE models are shown in Figure 5-5. The overall dimensions of the model are taken as $3 \mathrm{~m} \times 3 \mathrm{~m} \times t \mathrm{~m}$. The final stress results are found from this analysis and compared to the allowable values to determine if including PCM in a concrete wall is acceptable.

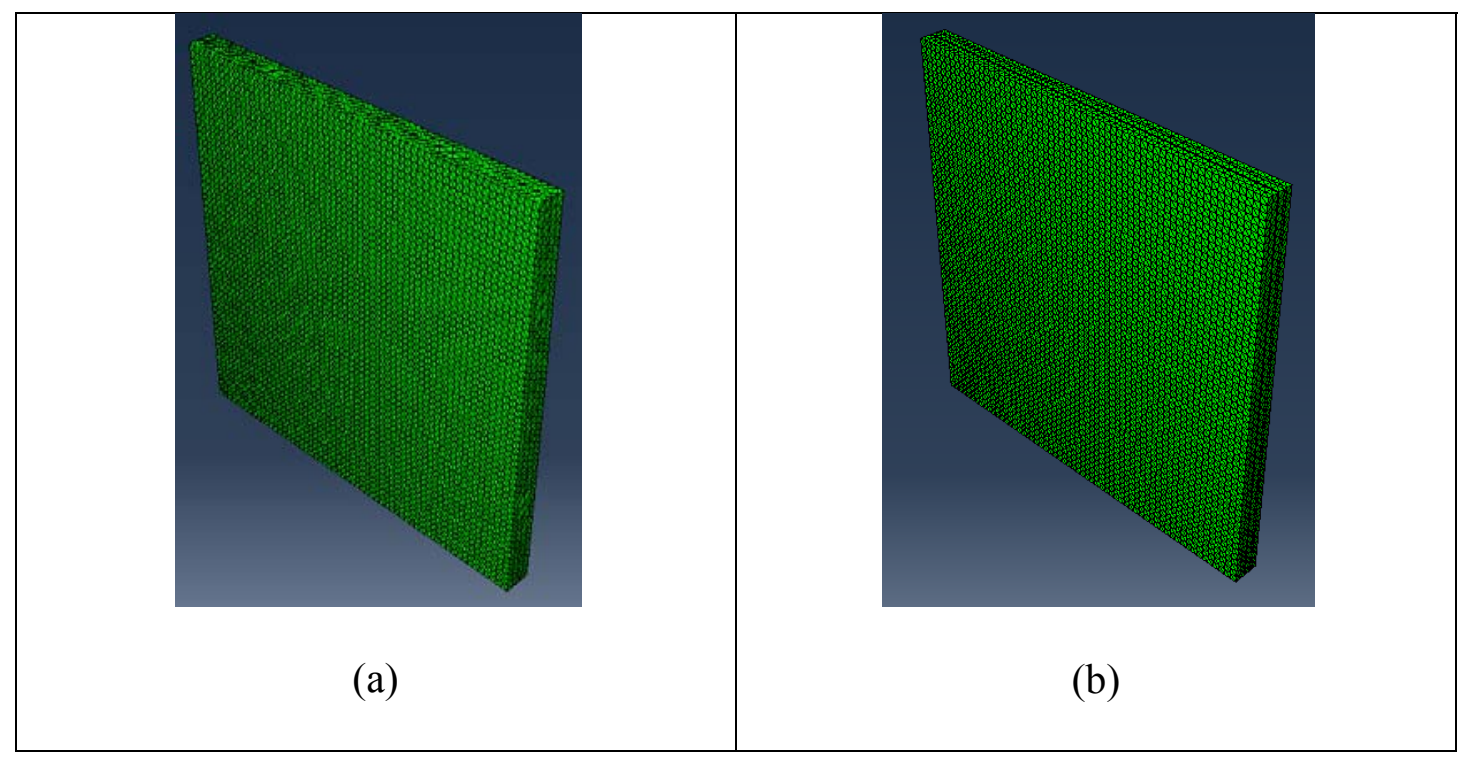

Figure 5-5: (a) 3LW structural FE model and (b) 5LW structural FE model

\subsection{Optimization of the Wall Designs}

The goal of the optimization process was to create the most cost effective wall by using a combination of PCM and concrete to minimize both the material and energy costs as well as meet structural performance and constructability requirements. Cost calculations are based on a hypothetical room with dimensions $l \times w \times h(l=w=h=3 m)$ with heat exchange taking place from five sides that contain the materials describing a typical wall configuration. No openings are assumed in any of the walls. The concrete and PCM thermal performance do not degrade over time and they require little or no maintenance. Therefore, the lifetime cost is calculated as the energy cost over a 10 year period plus the 
initial material costs only. We consider different annual cost escalation for the energy component of the total cost; a 1\% annual increase in energy cost is assumed along with a $5 \%$ annual increase in energy cost based on different inflation rates over the 10 year period.

The cost values are based on the following information:

1) The cost of normal weight concrete is $\$ 113.85 / \mathrm{m}^{3}$ [49].

2) The cost of the PCM varies based on the way it is manufactured (whether it is available in bulk form, or is microencapsulated) and the publicly available cost values are shown in Table 5-3. It is clear that the (initial) cost of PCM is extremely high compared to the concrete cost [50].

3) The cost of energy was found from APS's Residential Pricing Chart and is shown in Table 5-4. There is a $20 \%$ increase in the summer energy cost and a $10 \%$ increase in the fall/spring energy cost compared to the lowest cost that is available during the winter season [51].

Table 5-3: Material Cost

\begin{tabular}{|l|l|l|}
\hline Material & \multicolumn{1}{|c|}{$\begin{array}{c}\text { Estimated } \\
\mathbf{C o s t} \\
\mathbf{( \$ / \mathbf { m } ^ { 3 } )}\end{array}$} & $\begin{array}{c}\text { Normalized } \\
\mathbf{C o s t} \\
\mathbf{( \$ / \mathbf { m } ^ { 3 }}\end{array}$ \\
\hline Concrete & 113.85 & 1.0 \\
\hline PCMA & 5463.66 & 47.99 \\
\hline PCMB & 3792.34 & 33.31 \\
\hline PCMC & 3747.94 & 32.92 \\
\hline PCMD & 3792.34 & 33.31 \\
\hline PCME & 2645.87 & 23.24 \\
\hline PCMF & 3792.34 & 33.31 \\
\hline PCMG & 3792.34 & 33.31 \\
\hline
\end{tabular}


Table 5-4: Energy Costs in Phoenix, AZ

\begin{tabular}{|c|c|c|}
\hline Time of Year & $\begin{array}{c}\text { Energy Cost - Year } \mathbf{1} \\
(\mathbf{2 0 1 2}) \mathbf{( \$ / k W h )}\end{array}$ & Number of days/year \\
\hline Summer & 0.112 & 120 \\
\hline Winter & 0.0939 & 90 \\
\hline Spring/Fall & 0.1033 & 155 \\
\hline
\end{tabular}

The overall optimization problem can be stated as follows (units are $\mathrm{kg}, \mathrm{m}, \mathrm{s}, \mathrm{W}, \$$ ).

Find

$$
\mathbf{x}=\left\{b_{1}, \ldots, b_{n}, m_{1}, \ldots, m_{n}\right\}
$$

to minimize $\quad \bar{C}=(2 l h+2 w h+l w)\left(\left[c_{c} \sum_{j=1}^{c} b_{j}+c_{p} \sum_{j=c+1}^{c+p} b_{j}\right]+\left[\frac{\sum_{l=1}^{3}\left(E F T I F \cdot c_{e} \cdot t\right)_{l}}{3.6 \times 10^{6}}\right]\right)$

subject to

$$
\begin{aligned}
& 0.20 \leq \sum_{j=1}^{c} b_{j}+\sum_{j=c+1}^{c+p} b_{j} \leq 0.25 \\
& 0.02 \leq b_{j} \leq 0.10, \quad j=1, \ldots, c \\
& 0.005 \leq b_{j} \leq 0.10, \quad j=c+1, \ldots, c+p \\
& m_{j} \subset\left[m_{1}^{p}, m_{2}^{p}, \ldots, m_{8}^{p}\right] \quad j=1, \ldots, c+p \\
& \sigma_{\max }^{t} \leq 1.7 \mathrm{MPa}, \sigma_{\max }^{c} \leq 27.6 \mathrm{MPa}
\end{aligned}
$$

where: $\bar{C}$

$$
\text { material plus energy cost over a } 10 \text { year period }
$$

$$
\begin{array}{ll}
b_{j} & \text { thickness of the } \mathrm{j}^{\text {th }} \text { layer of the wall (concrete or PCM) } \\
c, p & \text { number of concrete and PCM layers, respectively } \\
c_{c}, c_{p c m}, c_{e} & \text { unit cost for concrete, PCM and energy } \\
m_{j} & \text { material for the } \mathrm{j}^{\text {th }} \text { layer of the wall } \\
n & \text { number of layers used in the wall } \\
t & \text { time multiplication factor }
\end{array}
$$


Eqn. 5.2 shows the potential design variables that are the material used in each layer and the thickness of each layer. However, as shown later, the material selection is restricted to certain layers only. The objective function (Eqn. 5.3) is computed as the sum of material cost and the 10-year energy cost. Eqn. 5.4 restricts the overall thickness of the wall to be between $0.20 \mathrm{~m}$ and $0.25 \mathrm{~m}$. Eqns. 5.5 and 5.6 show the range of permissible wall thicknesses for concrete and PCM respectively. Eqn. 5.7 shows that the material in each layer must be one of the 8 materials listed in Table 3A. Finally, Eqn. 5.8 restricts the maximum principal tensile stress and compressive stress to the specified values. The FE analysis for each wall was performed in ABAQUS for a total of 48 hours and the results of the energy flow for only the second 24 hours were considered in the objective function.

Both the $3 \mathrm{LW}$ and 5LW designs were optimized to determine the optimal wall configurations for each model based on the problem formulation discussed earlier. A two-stage optimization was carried out to better understand the use of different PCMs and the results of changing the layer thickness, in order to obtain the solution in a computationally efficient mode. Each model was run for 20 generations with a population size of 30 for a total of 600 function evaluations.

\subsubsection{Stage 1 Optimization (Material Selection)}

The primary goal was to select the appropriate material for the PCM layer(s). All seven PCM materials were considered as candidate materials for Layer 3 in the $3 \mathrm{LW}$ model, and layers 2 and 4 in the 5LW model. The results from this stage of the optimization are shown in Table 5 where the material-optimized models are compared against the concrete only design (NC Wall Model). Two cost models are considered for computing the energy 
cost - a $1 \%$ annual cost escalation and a $5 \%$ annual cost escalation. Arizona's annual residential electricity price over the last two decades shows an initial peak of about $\$ 0.097 / \mathrm{kWH}$ (1992-93), decreasing to about $\$ 0.084 / \mathrm{kWH}$ (2002) and thereafter, showing a rapid annual increase to a price of about $\$ 0.107 / \mathrm{kWH}$ (2009). The concrete and PCM thickness used were chosen based on previous studies done [35].

Table 5-5: Baseline Design Comparison

\begin{tabular}{|c|c|c|c|c|}
\hline & & $\begin{array}{c}\text { NC Wall } \\
\text { Model }\end{array}$ & $\begin{array}{c}\text { Material } \\
\text { Optimized } \\
\text { 3LW } \\
\text { Model } \\
\end{array}$ & $\begin{array}{c}\text { Material } \\
\text { Optimized } \\
\text { 5LW } \\
\text { Model }\end{array}$ \\
\hline \multirow[t]{2}{*}{ Layer 1} & Material & Concrete & Concrete & Concrete \\
\hline & $\begin{array}{l}\text { Thickness } \\
\text { (m) }\end{array}$ & 0.04 & 0.075 & 0.05 \\
\hline \multirow[t]{2}{*}{ Layer 2} & Material & Concrete & & PCMF \\
\hline & $\begin{array}{l}\text { Thickness } \\
\text { (m) }\end{array}$ & 0.04 & & 0.025 \\
\hline \multirow[t]{2}{*}{ Layer 3} & Material & Concrete & PCMD & Concrete \\
\hline & $\begin{array}{l}\text { Thickness } \\
\text { (m) }\end{array}$ & 0.04 & 0.05 & 0.05 \\
\hline \multirow[t]{2}{*}{ Layer 4} & Material & Concrete & & PCMD \\
\hline & $\begin{array}{l}\text { Thickness } \\
\text { (m) }\end{array}$ & 0.04 & & 0.025 \\
\hline \multirow[t]{2}{*}{ Layer 5} & Material & Concrete & Concrete & Concrete \\
\hline & $\begin{array}{l}\text { Thickness } \\
\text { (m) }\end{array}$ & 0.04 & 0.075 & 0.05 \\
\hline $\begin{array}{l}\text { Total Wall } \\
\text { thickness (m) }\end{array}$ & & 0.20 & 0.20 & 0.20 \\
\hline Material Cost (\$) & & $\$ 1,024.65$ & $\begin{array}{l}\$ 9,352.77 \\
(+813 \%)\end{array}$ & $\begin{array}{l}\begin{array}{l}\$ 9,300.38 \\
(+808 \%)\end{array} \\
\end{array}$ \\
\hline $\begin{array}{l}\text { Energy Cost } 1 \% \\
\text { cost escalation }(\$)\end{array}$ & & $\$ 13,946.84$ & $\begin{array}{l}\begin{array}{l}\$ 6,832.86 \\
(-51 \%)\end{array} \\
\end{array}$ & $\begin{array}{l}\$ 7,187.33 \\
(-49 \%)\end{array}$ \\
\hline $\begin{array}{l}\text { Energy Cost 5\% } \\
\text { cost escalation (\$) }\end{array}$ & & $\$ 16,767.19$ & $\begin{array}{l}\$ 8,214.61 \\
(-51 \%)\end{array}$ & $\begin{array}{l}\$ 8,640.76 \\
(-49 \%)\end{array}$ \\
\hline $\begin{array}{l}\text { Total Cost } 1 \% \\
\text { cost escalation }(\$)\end{array}$ & & $\$ 14,971.49$ & $\begin{array}{l}\$ 16,185.63 \\
(+8 \%)\end{array}$ & $\begin{array}{l}\$ 16,487.71 \\
(+10 \%)\end{array}$ \\
\hline $\begin{array}{l}\text { Total Cost } 5 \% \\
\text { cost escalation (\$) }\end{array}$ & & $\$ 17,791.84$ & $\begin{array}{l}\$ 17,567.38 \\
(-1.2 \%)\end{array}$ & $\begin{array}{l}\begin{array}{l}\$ 17,941.14 \\
(+0.8 \%)\end{array} \\
\end{array}$ \\
\hline
\end{tabular}


The results show that (a) a hot day PCM (PCMD) is the best PCM choice in the $3 \mathrm{LW}$ model, and (b) placing the cold day PCM (PCMF) closer to the exterior face of the wall and the hot day PCM (PCMD) closer to the interior face of the wall was the most efficient design for the 5LW model. The figures in parenthesis in the last two columns represent the cost increase (+) or cost decrease (-) with respect to the NC Wall Model. With these models, the NC Wall Model is the most cost efficient model when $1 \%$ annual cost escalation is considered, and the $3 \mathrm{LW}$ Model is the most cost efficient model when $5 \%$ annual cost escalation is considered. The initial PCM material cost is a huge component of the entire 10-year cost so an optimized design could result from a lower usage of PCM material and optimizing all the layer thicknesses. As discussed earlier, this scenario could be changed if the use of PCM becomes more accepted and manufacturers devise materials and methods to drive down the material cost.

\subsubsection{Stage 2 Optimization (Layer Thickness)}

The goal of this stage was to use the materials selected from stage 1 and to optimize all the layer thicknesses to get a final optimal design for both walls. As found from the Stage 1 Optimization, the PCM material selection for the 3LW was PCMD. The influence of the inflation rate on the energy cost was determined to be significant, so there were actually separate optimizations performed for the $1 \%$ inflation and $5 \%$ inflation rate using both GA and DE as the optimizers, for a total of four different optimizations on the 3LW. The results are summarized in the table below. 
Table 5-6: 3LW Optimized Designs

\begin{tabular}{|c|c|c|c|c|c|}
\hline & & $\begin{array}{c}1 \% \\
\text { Thickness } \\
\text { Optimized } \\
\text { 3LW-GA }\end{array}$ & $\begin{array}{c}1 \% \\
\text { Thickness } \\
\text { Optimized } \\
\text { 3LW-DE }\end{array}$ & $\begin{array}{c}5 \% \\
\text { Thickness } \\
\text { Optimized } \\
\text { 3LW-GA }\end{array}$ & $\begin{array}{c}5 \% \\
\text { Thickness } \\
\text { Optimized } \\
\text { 3LW-DE }\end{array}$ \\
\hline \multirow[t]{2}{*}{ Layer 1} & Material & Concrete & Concrete & Concrete & Concrete \\
\hline & $\begin{array}{l}\text { Thickness } \\
\text { (m) }\end{array}$ & 0.099 & 0.09928 & 0.099 & 0.09963 \\
\hline \multirow[t]{2}{*}{ Layer 3} & Material & PCMD & PCMD & PCMD & PCMD \\
\hline & $\begin{array}{l}\text { Thickness } \\
\text { (m) }\end{array}$ & 0.036 & 0.03504 & 0.039 & 0.03751 \\
\hline \multirow[t]{2}{*}{ Layer 5} & Material & Concrete & Concrete & Concrete & Concrete \\
\hline & $\begin{array}{l}\text { Thickness } \\
\text { (m) }\end{array}$ & 0.099 & 0.09951 & 0.099 & 0.09983 \\
\hline $\begin{array}{l}\text { Total Wall } \\
\text { thickness (m) }\end{array}$ & & 0.234 & 0.2338 & 0.237 & 0.2369 \\
\hline $\begin{array}{l}\text { Max. Tensile } \\
\text { stress (kPA) }\end{array}$ & & 175 & 174 & 180 & 183 \\
\hline $\begin{array}{l}\text { Max. } \\
\text { Compressive } \\
\text { stress (kPA) }\end{array}$ & & 474 & 471 & 480 & 484 \\
\hline $\begin{array}{l}\text { Material Cost } \\
\text { (\$) }\end{array}$ & & $\begin{array}{l}\$ 7,157.36 \\
(+598 \%)\end{array}$ & $\begin{array}{l}\$ 6,990.31 \\
(+582 \%)\end{array}$ & $\begin{array}{l}\$ 7,669.27 \\
(+648 \%)\end{array}$ & $\begin{array}{l}\$ 7,422.19 \\
(+624)\end{array}$ \\
\hline Energy Cost (\$) & & $\begin{array}{l}\$ 7,652.86 \\
(-45.1 \%)\end{array}$ & $\begin{array}{l}\$ 7,858.19 \\
(-43.66 \%)\end{array}$ & $\begin{array}{l}\$ 8,661.86 \\
(-48.3 \%)\end{array}$ & $\begin{array}{l}\$ 8,882.64 \\
(-47.0 \%)\end{array}$ \\
\hline Total Cost $(\$)$ & & $\begin{array}{l}\$ 14,810.22 \\
(-1.08 \%)\end{array}$ & $\begin{array}{l}\$ 14,848.50 \\
(-0.82 \%)\end{array}$ & $\begin{array}{l}\$ 16,331.13 \\
(-8.2 \%)\end{array}$ & $\begin{array}{l}\$ 16,304.83 \\
(-8.4 \%)\end{array}$ \\
\hline
\end{tabular}

As Table 5-6 shows, the different optimizations for the $3 \mathrm{LW}$ all produced a similar wall layout for each of the four optimizations performed, showing that this layout is the only optimum for the 3LW. Again, the percents placed in parenthesis represent the cost increase (+) or decrease (-) when compared to the $\mathrm{NC}$ wall model. The optimization process increased the concrete content in the wall (from $0.15 \mathrm{~m}$ to about $0.198 \mathrm{~m}$ ) and decreased the PCM content (from $0.05 \mathrm{~m}$ to $0.036 \mathrm{~m}$ or $0.039 \mathrm{~m}$ ). The total cost decreased nominally from $\$ 16,185.63$ to about $\$ 14,800$ (1\% cost escalation) and from $\$ 17,567.38$ to around $\$ 16,300$ (5\% cost escalation). As stated earlier, the amount of 
inflation plays a major role in the optimization because when there is a $5 \%$ inflation rate for energy cost the savings of the total cost is much greater than that of the $1 \%$ inflation rate.

Table 5-7: 5LW Optimized Designs

\begin{tabular}{|c|c|c|c|c|c|}
\hline & & $\begin{array}{c}1 \% \\
\text { Thickness } \\
\text { Optimized } \\
\text { 5LW-GA }\end{array}$ & $\begin{array}{c}1 \% \\
\text { Thickness } \\
\text { Optimized } \\
\text { 5LW-DE }\end{array}$ & $\begin{array}{c}5 \% \\
\text { Thickness } \\
\text { Optimized } \\
\text { 5LW-GA }\end{array}$ & $\begin{array}{c}5 \% \\
\text { Thickness } \\
\text { Optimized } \\
\text { 5LW-DE }\end{array}$ \\
\hline \multirow[t]{2}{*}{ Layer 1} & Material & Concrete & Concrete & Concrete & Concrete \\
\hline & $\begin{array}{l}\text { Thickness } \\
\text { (m) }\end{array}$ & 0.085 & 0.05104 & 0.085 & 0.09891 \\
\hline \multirow[t]{2}{*}{ Layer 2} & Material & PCMF & PCMF & PCMF & PCMF \\
\hline & $\begin{array}{l}\text { Thickness } \\
\text { (m) }\end{array}$ & 0.005 & 0.00504 & 0.005 & 0.00501 \\
\hline \multirow[t]{2}{*}{ Layer 3} & Material & Concrete & Concrete & Concrete & Concrete \\
\hline & $\begin{array}{l}\text { Thickness } \\
\text { (m) }\end{array}$ & 0.085 & 0.09999 & 0.085 & 0.09851 \\
\hline \multirow[t]{2}{*}{ Layer 4} & Material & PCMD & PCMD & PCMD & PCMD \\
\hline & $\begin{array}{l}\text { Thickness } \\
\text { (m) }\end{array}$ & 0.023 & 0.005 & 0.023 & 0.02488 \\
\hline \multirow[t]{2}{*}{ Layer 5} & Material & Concrete & Concrete & Concrete & Concrete \\
\hline & $\begin{array}{l}\text { Thickness } \\
\text { (m) }\end{array}$ & 0.05 & 0.08891 & 0.05 & 0.02243 \\
\hline $\begin{array}{l}\text { Total Wall } \\
\text { thickness (m) }\end{array}$ & & 0.248 & 0.2499 & 0.248 & 0.2497 \\
\hline $\begin{array}{l}\text { Max. Tensile } \\
\text { stress (kPA) }\end{array}$ & & 144 & 119 & 144 & 141 \\
\hline $\begin{array}{l}\text { Max. } \\
\text { Compressive } \\
\text { stress (kPA) }\end{array}$ & & 297 & 381 & 297 & 283 \\
\hline $\begin{array}{l}\text { Material Cost } \\
(\$)\end{array}$ & & $\begin{array}{l}\$ 5,904.97 \\
(+476 \%)\end{array}$ & $\begin{array}{l}\$ 2,943.55 \\
(+\mathbf{1 8 7 \%})\end{array}$ & $\begin{array}{l}\$ 5,904.97 \\
(+476 \%)\end{array}$ & $\begin{array}{l}\$ 6,288.23 \\
(+513)\end{array}$ \\
\hline Energy Cost (\$) & & $\begin{array}{l}\$ 8,105.14 \\
(-41.9 \%)\end{array}$ & $\begin{array}{l}\$ 10,964.10 \\
(-21.4 \%)\end{array}$ & $\begin{array}{l}\$ 9,744.17 \\
(-41.9 \%)\end{array}$ & $\begin{array}{l}\$ 9,210.75 \\
(-45.1 \%)\end{array}$ \\
\hline Total Cost (\$) & & $\begin{array}{l}\$ 14,010.11 \\
(-6.42 \%)\end{array}$ & $\begin{array}{l}\$ 13,907.65 \\
(-7.11 \%)\end{array}$ & $\begin{array}{l}\$ 15,649.14 \\
(-12.0 \%)\end{array}$ & $\begin{array}{l}\$ 15,498.98 \\
(-12.9 \%)\end{array}$ \\
\hline
\end{tabular}

As Table 5-7 shows, the results for the 5LW did not produce similar layouts for each of the four optimizations showing there are multiple optimums for this design. The use of 
GA resulted in the exact same layout for both the $1 \%$ and $5 \%$ energy cost inflation, but DE optimized differently for each inflation rate. For the $1 \%$ rate, the DE optimizer found the minimum cost using hardly any PCM, only 4\% of the wall was PCM. This is the result of how significant the initial material cost is for PCM. For the 5\% rate, the DE optimizer found a wall layout similar to that of the layouts found using GA. The difference in the optimal designs is the result of the different population manipulations the two optimizers use. It shows that there may be multiple designs that provide a minimum solution; it just depends on the input parameters and method of optimization. However, the final cost values for GA and DE are almost the same amount for the respective inflation rates. Again, the optimization process increased the concrete content in each wall and decreased the PCM content due to the large material cost caused by adding PCM. The total cost decreased nominally from $\$ 16,487.71$ to about $\$ 14,000$ (1\% cost escalation) and from $\$ 17,941.14$ to around $\$ 15,500$ (5\% cost escalation). As these results show, when compared to the $3 \mathrm{LW}$, the $5 \mathrm{LW}$ is a more cost effective wall model for both energy cost situations.

From all these optimizations performed, only one of the thickness optimized 3LW and 5LW models were selected to study further. The models that provided the most energy reduction using the 5\% cost increase were selected, which is the GA optimized 3LW and DE optimized 5LW. For the 3LW this represents: 0.099 concrete -0.039 PCMD -0.099 concrete. For the 5LW the layout is: 0.099 concrete -0.005 PCMF -0.099 concrete 0.025 PCMD -0.022 concrete.

Figure 5-6 shows the change of the temperature on the inside face of the wall for the different wall configurations: NC Wall, 3LW and 5LW; along with the ambient 
temperature conditions for the three different temperature cycles. The results show that the wall with no PCM had an inside wall temperature very closely correlated to the outside ambient temperature. For the summer day, the thickness optimized $3 \mathrm{LW}$ is better at keeping the temperature closer to the inside ambient temperature. This is due to thicker layer of PCMD that the $3 \mathrm{LW}$ contains compared to the $5 \mathrm{LW}$. For the winter and spring day, both thickness optimized designs regulate the temperature of the wall fairly the same. The use of PCMF in the 5LW is so thin it does not offer very much temperature regulation during a winter day. The $5 \mathrm{LW}$ model showed to be more economical because even though a smaller amount of PCM was used, the inside wall temperature is regulated much better than not using any PCM, but the material cost is much lower. As a result of better temperature regulation on the inside face of the wall, the indoor ambient temperature will stay much closer to the comfortable range with much less work from an HVAC system, thus the reduction in energy cost happens. 


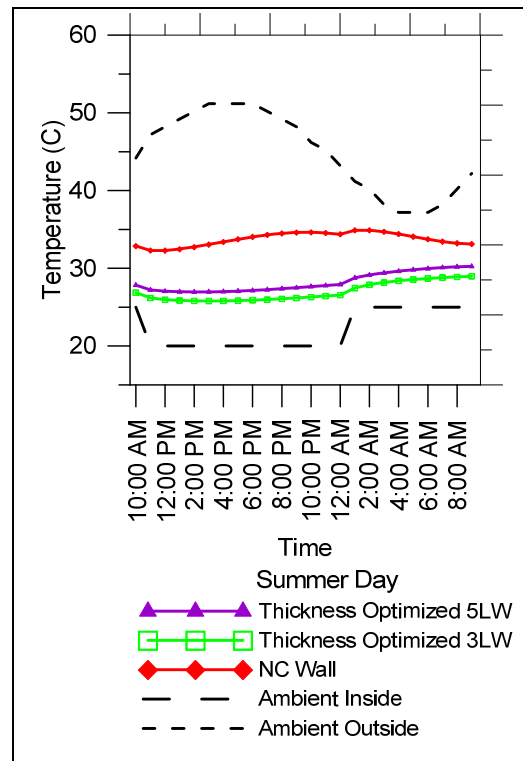

(a)

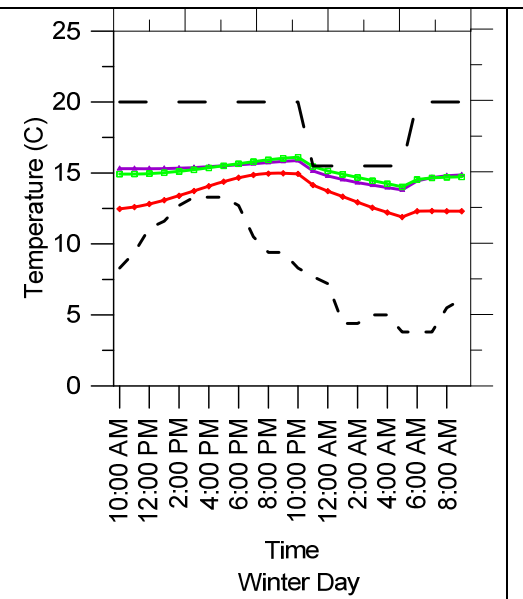

Winter Day

$\longrightarrow$ Thickness Optimized 5LW

$\square \square \square$ Thickness Optimized 3LW

$\longrightarrow$ NC Wall

- - Ambient Inside

- _ - Ambient Outside

(b)

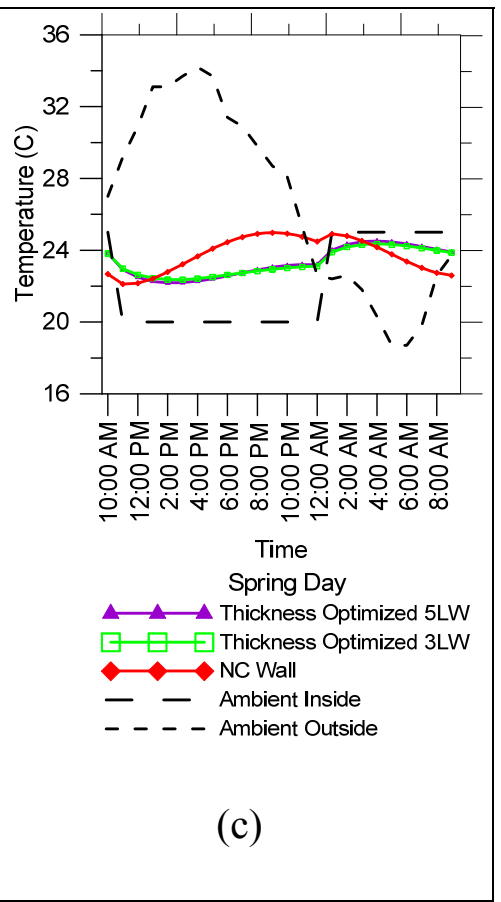

Figure 5-6: Temperature variation as a function of time on the inside face of the wall (a) Summer Day (b) Winter Day (c) Spring/Fall Day

Figure 5-7 shows the energy flow through the inner face of the wall for the same three models. These energy flow graphs show a similar trend as seen in the temperature graphs.

The wall with no PCM is much less energy efficient than a wall with PCM for all the temperature conditions. The summer graph shows the largest difference in energy flow between the $3 \mathrm{LW}$ and 5LW models, but this is again due to the $28 \%$ reduction in PCMD thickness in the 5LW. This is also apparent when looking at the above tables, as the $3 \mathrm{LW}$ has lower energy costs for all optimizations with much higher material costs. 


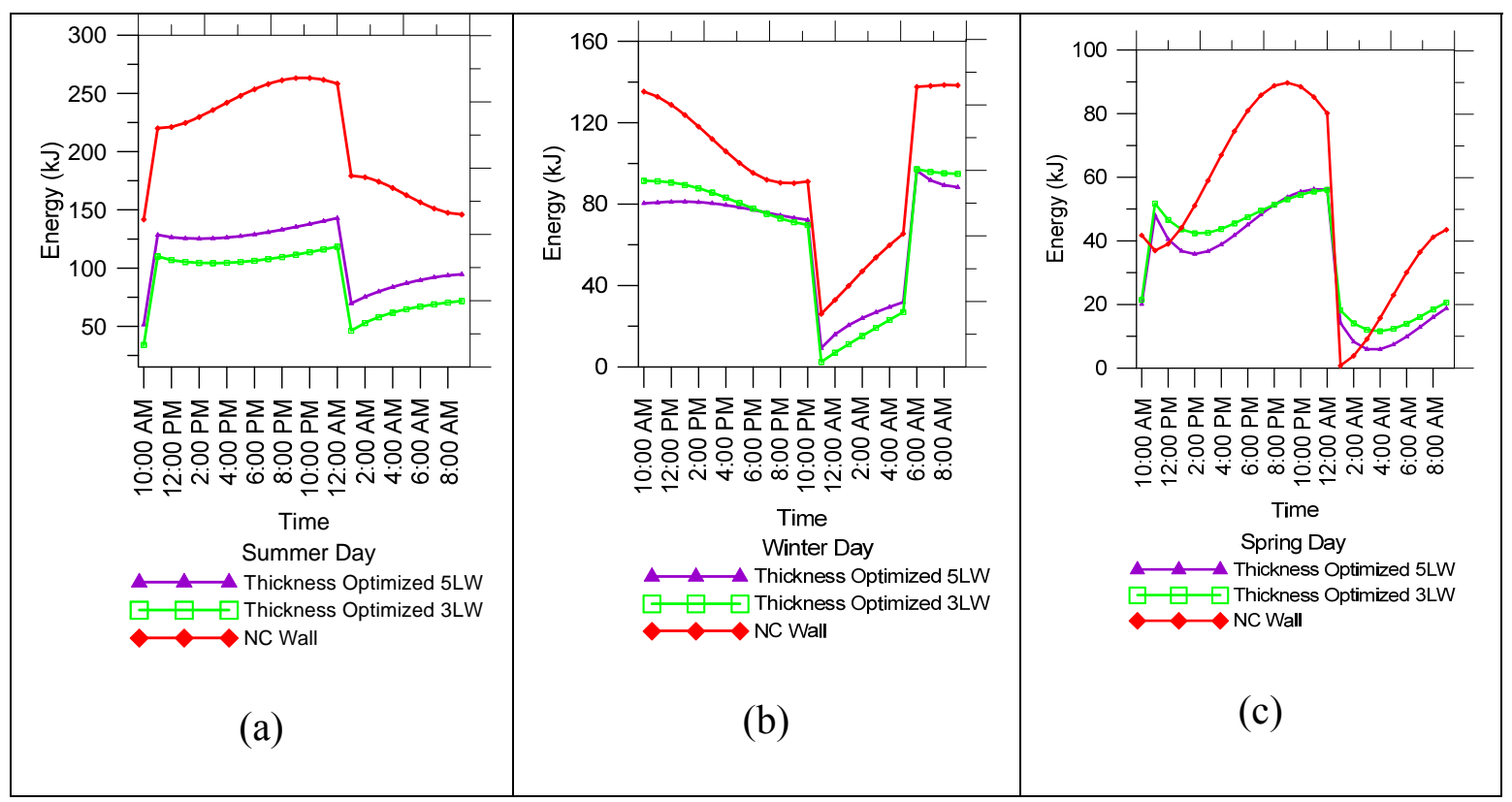

Figure 5-7: Energy flow through the inner face with respect to time (a) Summer Day (b) Winter Day (c) Spring/Fall Day

Figure 5-8(a) shows the temperature profile across the concrete only wall for the summer day only. The blue line on each graph represents the temperature across the wall at the minimum temperature for that day while the red line represents the temperature across the wall at the maximum temperature for that day. Figure 5-8(b) shows the temperature variation for the thickness optimized 3LW design. Figure 5-8(c) represents the thickness optimized 5LW design. As the graphs show the walls that contain PCM regulate the temperature much closer to the indoor temperature of $20-25^{\circ} \mathrm{C}$ than the NC Wall. 


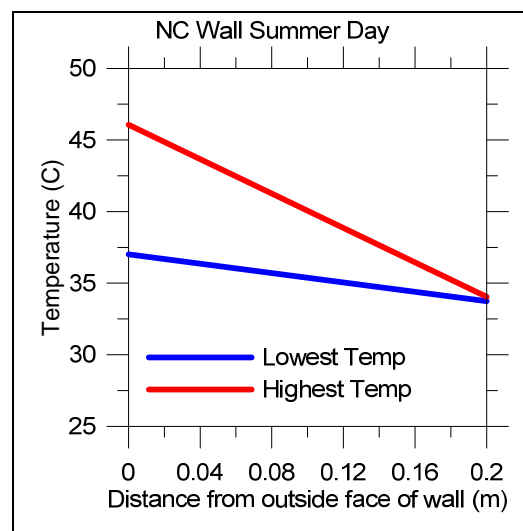

(a)

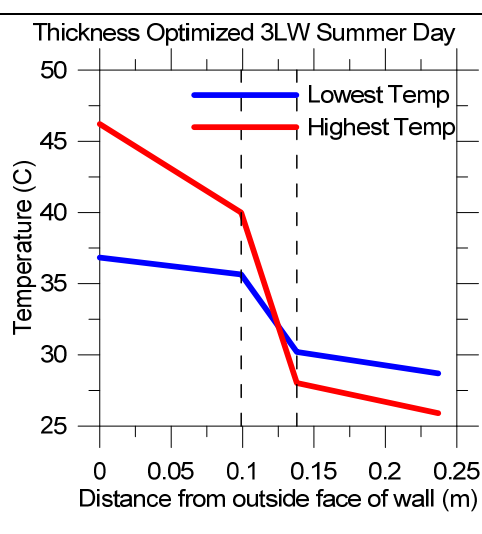

(b)

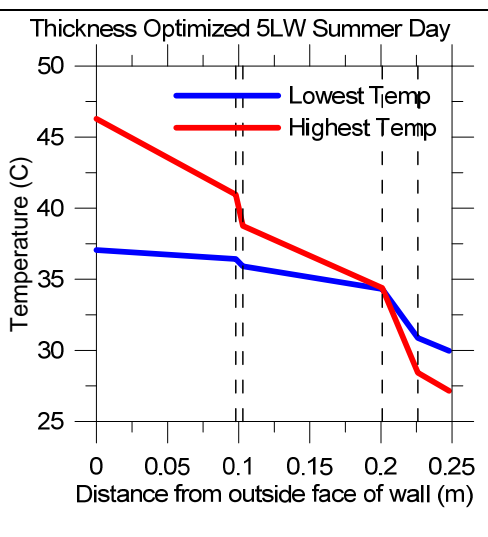

(c)

Figure 5-8: Temperature across the wall (a) Normal Concrete Wall, (b) Thickness Optimized 3LW and (c) Thickness Optimized 5LW

The results of the five layer model showed a clear benefit for use over the three layer model, and the efficiency of the PCM layers in each model show these benefits. Figure 12 shows the efficiency of all the models for the summer day and the winter day. For the spring day, none of the PCM's latent heat capability was used, so there is zero efficiency for every layer. The graphs show that the $5 \mathrm{LW}$ model is using both layers of PCM much more effectively throughout a year, and thus adds to the overall cost reduction during the entire year and not just for one season. 


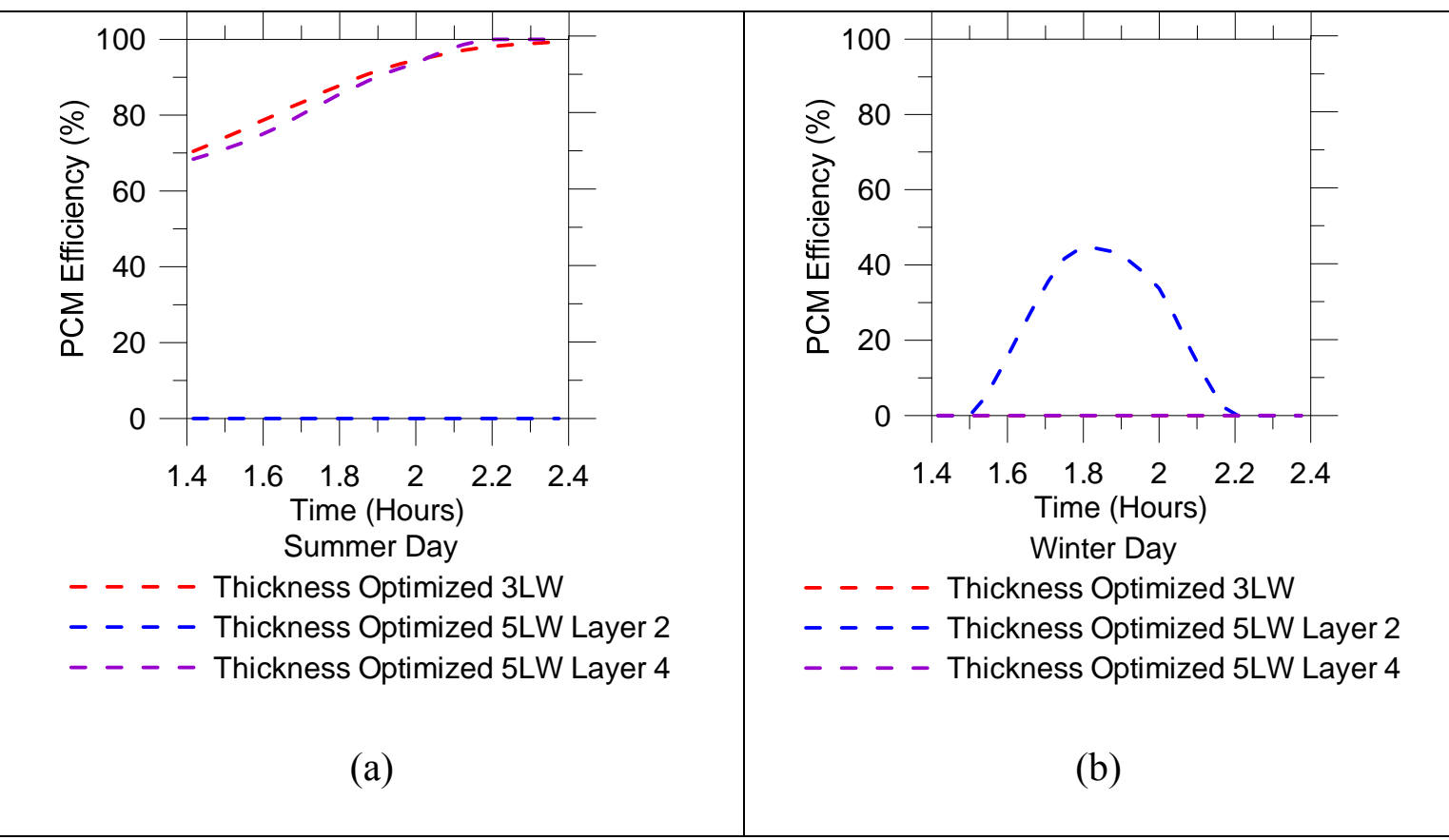

Figure 5-9: PCM Efficiency (a) Summer Day, (b) Winter Day, (c) Spring/Fall Day

The efficiency of every PCM layer varied by the wall configuration and the type of day it was, and can be summarized below.

Summer Day efficiency:

- The PCM layer in the thickness optimized 3LW (Layer 3) has a similar efficiency as Layer 4 in the thickness optimized 5LW.

- The Layer 4 in the $5 \mathrm{LW}$ would be expected to have higher efficiency because of the smaller volume of PCM in the wall, but the placement is much closer to the inside face of the wall so the temperatures acting on the PCM are lower during the summer day than those on the PCM layer in the $3 \mathrm{LW}$. This is also shown in Figure 5-8.

Winter Day efficiency:

- Only Layer 2 in the $5 \mathrm{LW}$ model is used for this temperature profile, the $3 \mathrm{LW}$ only contains a PCM good for summer days. 
- The cold day PCM reaches about 50\% efficiency during this day which provides some temperature regulation.

Spring Day efficiency:

- There is no PCM used for its latent energy capacity during this day because the temperatures never reach the phase change range of either PT-27 or PT-12.

- EFTIF was still less for the walls containing PCM on the spring day and that is the result of the PCM providing a better thermal mass layer than the concrete alone because of the larger sensible heat capacity that PCM has over concrete.

\subsection{Stress Analysis}

The results from the stress analysis are shown in Figure 5-10. The maximum tensile and compressive stresses on both the $3 \mathrm{LW}$ and 5LW are significantly lower than the allowable values $\left(\sigma_{\mathrm{aT}}=1700 \mathrm{kPA}, \sigma_{\mathrm{aC}}=27000 \mathrm{kPA}\right)$. The maximum stresses are at the supports as shown in the following Figure. The 3LW Models have higher stress values than the 5LW Model because a larger amount of the wall contains PCM - 16\% of the $3 \mathrm{LW}$ is PCM whereas only $11 \%$ of the $5 \mathrm{LW}$ is PCM, so there is less structural material used. However, even with this increase in PCM, the stresses are still very small. 


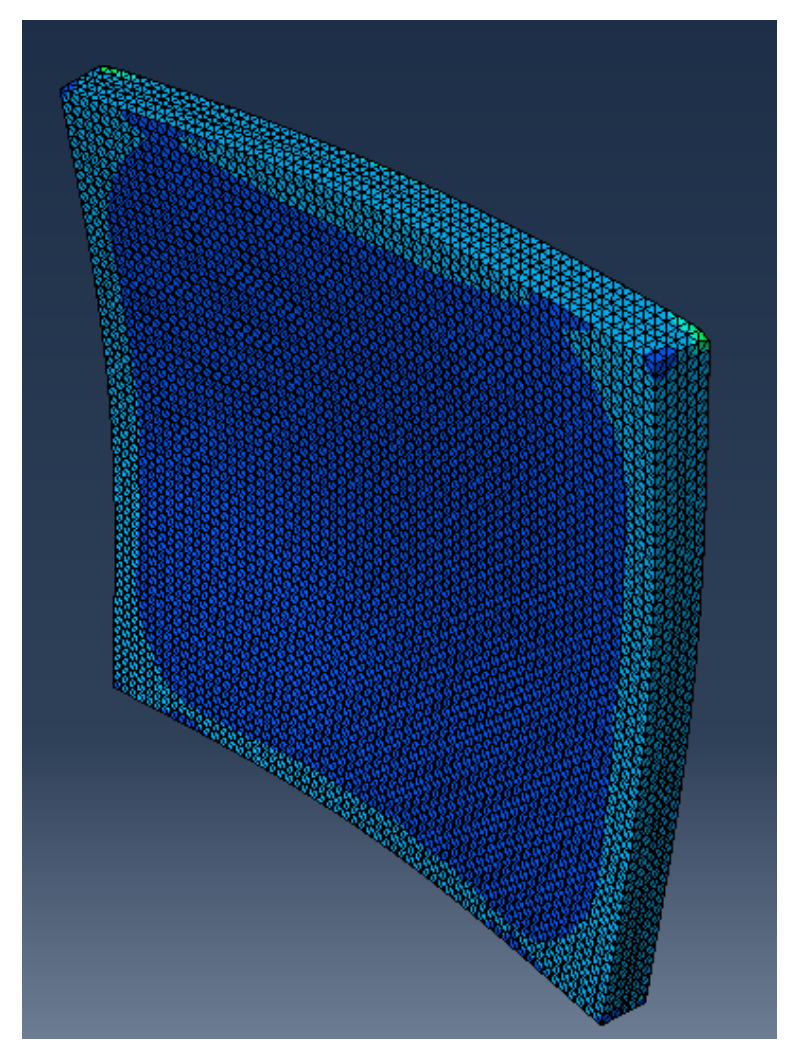

Figure 5-10: Maximum (Tensile) Principal Stress distribution

\subsection{Comparison of IBVP Results to ABAQUS}

To ensure the IBVP Program was providing correct results, the final optimized designs were also ran through the ABAQUS FEA and the results were compared. The comparisons will be done based on the EFTIF values only because material cost does not change between the two FEA programs. The following table shows the EFTIF values that have already been converted to an energy cost, found from both programs and the percent error between the values. 
Table 5-8: Comparison of IBVP program to ABAQUS EFTIF values

\begin{tabular}{|c|c|c|c|}
\hline Wall Design & IBVP EFTIF (\$) & $\begin{array}{c}\text { ABAQUS EFTIF } \\
(\$)\end{array}$ & Error (\%) \\
\hline 1\% 3LW GA & $\$ 7,652.86$ & $\$ 7,706.87$ & 0.70 \\
\hline 1\% 3LW DE & $\$ 7,858.19$ & $\$ 7,856.64$ & -0.02 \\
\hline $5 \%$ 3LW GA & $\$ 8,661.68$ & $\$ 8,717.73$ & 0.64 \\
\hline 5\% 3LW DE & $\$ 8,882.64$ & $\$ 8,935.33$ & 0.58 \\
\hline 1\% 5LW GA & $\$ 8,105.14$ & $\$ 8,194.31$ & 1.08 \\
\hline 1\% 5LW DE & $\$ 10,964.10$ & $\$ 11,179.54$ & 1.92 \\
\hline 5\% 5LW GA & $\$ 9,744.17$ & $\$ 9,852.05$ & 1.09 \\
\hline $5 \%$ 5LW DE & $\$ 9,210.75$ & $\$ 9,278.49$ & 0.73 \\
\hline
\end{tabular}

As Table 5-8 shows, there is only a maximum of $2 \%$ error between the energy flow calculations. This is most likely due to the round off difference between the programs and the way the mesh is created for each. However, because the error is so minimal it can be concluded that the IBVP program provides a framework with satisfactory results when performing FEA on phase change materials.

\subsection{Concluding remarks}

An optimal design methodology was developed for optimizing a wall containing both PCM and concrete. The design goal was taken as material and energy cost for a specified lifespan (10 years) using a specified annual energy cost escalation figure (1\% and 5\%) while preserving the structural performance. Different climatic conditions (spring/fall, summer and winter days) are considered in computing the energy cost. While it is difficult to manually configure a typical wall for the lowest total cost, the computational framework provided an automated tool for searching for the best design that balances material and energy costs. The optimization process is carried out with as realistic, publicly available cost figures as possible. It should be noted that the focus of the research work was to develop and implement a general framework under which the wall 
optimization could be carried out with different input parameter values - cost figures, available material and local climatic conditions.

The major findings in this study are summarized below.

(i) The design optimization framework helps determine the thickness and location of PCM and concrete for a given temperature profile. The use of PCM as a thermal layer in a wall is beneficial to regulate energy flow and energy costs in a building, but by finding the optimum material, location and thickness values creating the most effective system it is possible to reduce the overall cost.

(ii) Current cost figures show that PCM is between 20 and 50 times the cost of normal concrete. Even with this enormous cost difference, it is possible to reduce the overall cost by a judicious mix and placement of concrete and PCM layers.

(iii) A five layer wall model is more cost effective than a three layer wall. This is due to the main benefit of using the two PCM layers for different temperature conditions that is seen in a place like Phoenix. It is not clear whether the ease of constructing the three layer wall is the same as a five layer wall.

(iv) It is expected that an increase in demand for more energy efficient buildings will lead to an increase in demand for economical PCM. Hence it is expected that development of high efficiency-low cost PCM will take place with increased vigor. 


\section{CONCLUSIONS}

The development of this program has enabled for efficient computation and optimization of walls that contain PCM. The final program allows for studying the thermal efficiency of these types of wall systems with the added capacity to manipulate the design. Previous to the development of this program, the finite element analysis was performed using ABAQUS, which took approximately $70 \mathrm{~s}$ (for a 48 hour simulation on a Pentium 4 CPU 3.00 GHz Workstation running Windows 7). Once the IBVP FEA was incorporated into the WallDesign program the analysis took about $8 \mathrm{~s}$ on the same computer, cutting the compute time significantly. Prior to the integration of the IBVP program, the time to perform the optimization of 600 iterations for the $3 \mathrm{LW}$ was approximately 40 hours and for the 5LW was approximately 42 hours (for the 3 temperature cycles, 48 hour simulation). The addition of IBVP cut this time to about 7 hours for the $3 \mathrm{LW}$ optimization and 9 hours for the 5LW optimization. This cuts computation time by $80 \%$. Also, both the GA and DE optimizer run close to the same amount of time, leaving it to the designer to choose the best optimizer for the design problem.

As a result, different effects of design parameters can be quickly studied and varied. These parameters can include wall thickness, material types, PCM types, layer location, boundary conditions and many other design parameters. The designer can vary any parameter effortlessly through the input file.

The major findings from this study are summarized below:

I. The finite element method provides a very powerful framework to analyze a PCM embedded wall system. The addition of phase change materials into these wall systems causes the FEA to become nonlinear because PCMs have a latent heat 
capacity during the phase change range. Since the FEM allows for use of nonlinear transient analysis and the ability to implement it in a computer program made it the best choice to analyze these building elements.

II. The use of optimization is also a very powerful tool, but a very time consuming process to gain any meaningful results. The automation of optimization allows the process to happen very quickly producing converging and significant results.

III. For the design problems that were considered, Genetic Algorithms and Differential Evolution were the best optimizers to use. They allow the use of both discrete and continuous design variables so the optimizations performed in this study could manipulate any model.

IV. All the tests cases that were performed and the comparing of the IBVP program to ABAQUS and the UNAFEM program validates the use of IBVP as an FEA program capable of performing linear and nonlinear transient analysis using $\mathrm{C}^{0}$ linear elements.

V. There are many different PCMs being manufactured today and based on the varying properties the optimizer can select the PCM best suited and most cost effective for the temperature conditions placed on the wall.

VI. The use of PCM in concrete walls can significantly reduce the amount of energy flow through the wall along with providing much better temperature regulation inside a room. The amount of the reduction is based on how much of the wall is actually the PCM. However, the addition of this material is very costly, so optimization can be used to find the best PCM thickness based on cost. The 
structural integrity of concrete walls does not decrease much with the addition of PCM.

VII. The use of multiple PCMs in a wall provides a better design than the use of only a single layer of PCM in a wall. This is due to the varying temperatures that most places have throughout the year, and using multiple PCM layers can provide a PCM with a phase change range that works for different seasons.

\subsection{Future Work}

The program developed deals with very specific cases but can be advanced in the future to deal with more wide ranging wall problems. The program can be increased to perform a $2 \mathrm{D}$ or even $3 \mathrm{D}$ analysis with very little effort. The incorporation of those analyses will allow for the inclusion of wall openings such as windows or doors, allowing for a more realistic model. Structural analysis could also be integrated into the finite element analysis allowing the program to be able to analyze both the thermal and structural effects of walls that contain PCM and be able to optimize the walls to have the best thermal properties without sacrificing the structural capacity.

The optimization portion could be developed to be performed in parallel which would increase computation time. Then a single wall optimization could be performed in a matter of minutes. 


\section{References}

[1] Federal Research and Development Agenda for Net Zero Energy, High Performance Green Buildings, National Science and Technology Council (NSTC) (2008) 60

[2] H. Mehling and L. F. Cabeza, Heat and cold storage with PCM, Springer-Verlag, Berlin (2008)

[3] F. Demirbas, Thermal energy storage and phase change materials: an overview. Energy Sources, Part B: Econ Plann Policy 1 (2006) 85-95

[4] N. Neithalath, G. Sant, Phase change materials in concrete: A new strategy to improve the thermal damage resistance and thermal energy efficiency of concrete structures, (unpublished results)

[5] F. Kuznik, D. David, K. Johannes and J. J. Roux, A review of PCM integrated in building walls, Renewable and Sustainable Energy Reviews 15 (1) (2011) 379-391

[6] A. Pasupathy, L. Athanasius, R. Velraj and R.V. Seeniraj, Experimental Investigation and numerical simulation analysis on the thermal performance of a building roof incorporating phase change material (PCM) for thermal management, Applied Thermal Engineering 28 (5-6) (2008) 556-565

[7] C.K. Halford and R.F. Boehm, Modeling of phase change material peak load shifting, Energy and Buildings, 39(3) (2007) 298-305

[8] B.M. Diaconu and M. Cruceru, Novel concept of composite phase change material wall system for year-round thermal energy savings, Energy and buildings, 42 (10) (2010) $1759-1772$

[9] K. Darkwa and P.W. O'Callaghan, Simulation of phase change drywalls in a passive solar building, Applied Thermal Engineering 26(8-9) (2006) 853-858 
[10] H. E. Feustel and C. Stetiu, Thermal Performance of Phase Change Wallboard for Residential Cooling Application, Lawrence Berkeley National Laboratory, Berkeley, CA (1997)

[11] A. Carbonari, M. De Grassi, C. Di Perna and P. Principi, Numerical and experimental analyses of PCM containing sandwich panels for prefabricated walls, Energy and Buildings 38(5) (2006) 472-483.

[12] F. Kuznik, J. Virgone, and J. Noel, Optimization of a phase change material wallboard for building use, Applied Thermal Engineering, 28(11-12) (2008) 1291-1298

[13] A. Sharma, V.V. Tyagi, C.R. Chen, D. Buddhi, Review on thermal energy storage with phase change materials and applications Renewable and Sustainable Energy Reviews, 13 (2) (2009) 318-345

[14] K. Darkwa and P.W. O'Callaghan, Simulation of phase change drywalls in a passive solar building, Applied Thermal Engineering 26(8-9) (2006) 853-858

[15] C. Zhang, Y. Chen, L. Wu and M. Shi, Thermal response of brick wall filled with phase change materials (PCM) under fluctuating outdoor temperatures, Energy and Buildings 43(12) (2011) 3514-3520

[16] D. Zhang, Z. Li, J. Zhou, K. Wu, Development of thermal energy storage concrete, Cement and concrete research, 34(6) (2004) 927-934

[17] A. Vaz Sa, M. Azenha, H. de. Sousa and A. Samagaio, Thermal enhancement of plastering mortars with Phase Change Materials: Experimental and numerical approach, Energy and Buildings, 49 (2012) 16-27

[18] F. Kuznik, D. David, K. Johannes and J. J. Roux, A review of PCM integrated in building walls, Renewable and Sustainable Energy Reviews 15 (1) (2011) 379-391 
[19] H. Zhang, Q. Xu, Z. Zhao, J. Zhang, Y. Sun, L. Sun, F. Xu, Y. Sawada, Preparation and thermal performance of gypsum boards incorporated with microencapsulated phase change materials for thermal regulation, Solar Energy Materials and Solar Cells 102 (2012) 93-102

[20] S. Rajan, Finite Elements for Engineers: Module 1 and 2, (2012)

[21] R. Lewis, The Finite Element Method in Heat Transfer Analysis, John Wiley \& Sons, England (1996)

[22] K. Bathe, and M. Khoshgoftaar, Finite Element Formulation and Solution of Nonlinear Heat Transfer, Nuclear Engineering and Design, 51 (1979) 389-401

[23] E. Wilson, K. Bathe, and F. Peterson, Finite Element Analysis of Linear and Nonlinear Heat Transfer, Nuclear Engineering and Design, 29 (1974) 110-124

[24] J. Reddy, Introduction to Nonlinear Finite Element Analysis, Oxford University Press, 61-85

[25] B. Nedjar, An Enthalpy Based Finite Element Method for Nonlinear Heat Problems Involving Phase Change, Computers and Structures, 80 (2002) 9-21

[26] V. Voller, Fast Implicit Finite Difference Method for the Analysis of Phase Change Problems, Numerical Heat Transfer, 17 (1990) 155-169

[27] Brief Overview of Numerical Methods for Phase Change Problems, Chapter 4, 210 223

[28] Y. Dutil, D. Rousse, N. Salah, S. Lassue, and L. Zalewski, A Review on Phase Change Materials: Mathematical Modeling and Simulations, Renewable and Sustainable Energy Reviews, 15 (2011) 112-130 
[29] M. Costa, D. Buddhi, and A. Oliva, Numerical Simulation of a Latent Heat Thermal Energy Storage System with Enhanced Heat Conduction, Energy Convers. Mgmt, 39 (1998) 319-330

[30] P. Lamberg, R. Lehtiniemi, and A. Henell, Numerical and Experimental Investigation of Melting and Freezing Processes in Phase Change Material Storage, International Journal of Thermal Sciences, 43 (2004) 277-287

[31] Q. Pham, The Use of Lumped Capacitance in the Finite Element Solution of Heat Conduction Problems with Phase Change, International Journal of Heat Mass Transfer, 29 (1986) 285-291

[32] V. Voller and C. Swaminathan, General Source Based Method for Solidification Phase Change, Numerical Heat Transfer, 19 (1991) 175-189

[33] ABAQUS v. 610 Theory Manual

http://abaqus.civil.uwa.edu.au:2080/v6.10/books/stm/default.htm (accessed on January 2013)

[34] S. Rao, Engineering Optimization Theory and Practice, 2010

[35] L. Hembade, A Finite Element Based Framework for Understanding the Energy Performance of Concrete Elements Incorporating Phase Change Materials, 2012

[36] Wall Panel Design Data, Hanson Heidelberg Cement Group, 2008 1-19

[37] T. Hassan and S. Rizkalla, Analysis and Design Guidelines of Precast, Prestressed Concrete, Composite Load Bearing Sandwich Wall Panels reinforced with CFRP Grid, PCI Journal, (2012) 147-162

[38] T. Shugar, Preliminary Analysis of Reinforced Concrete Waffle Walls, Naval Facilities Engineering Service Center (1997) 
[39] B. Frankly, Structural Behavior of Insulated Precast Prestressed Concrete Sandwich Panels Reinforced with CFRP Grid, 2008

[40] D. Burnett, Finite Element Analysis From Concepts to Applications, AddisonWesley Publishing, 1987

[41] S. Enes, Shape Functions Generation, Requirements, Etc., Ruhr University Bochum, 2009

[42] C. Fonseca and P. Fleming, An Overview of Evolutionary Algorithms in Multiobjective Optimization, The University of Sheffield, 1995

[43] R. Storn and K. Price, Differential Evolution- A Simple and Efficient Heuristic for Global Optimization over Continuous Spaces, Journal of Global Optimization, 11 (1997) 341-359

[44] K. Price and R. Storn, Differential Evolution for Continuous Function Optimization, http://www1.icsi.berkeley.edu/ storn/code.html

[45] S. Rajan, EDO User's Manual, Arizona State University, 2011

[46] The Weather Channel, www.weather.com

[47]The Weather Underground Inc, www.wunderground.com

[48]Y. Lee, M.S. Choi, S.T. Yi and J.K. Kim, Experimental study on the convective heat transfer coefficient of early-age concrete, Cement and Concrete Composites 31(1) (2009) $60-71$

[49] Reed Construction Data. (2009). RS Means Building Construciton Cost Data.

[50] K. Roth, D. Westphalen, J. Dieckman, S. Hamilton, and W. Goetzler, Energy Consumption Characteristics of Commercial Building HVAC Systems Volume III. Building Technologies Program, A-108 (2002) 
[51] APS Residential Pricing Chart (2012) Retrieved from APS.

http://www.aps.com/main/services/residential/rates/rates_29.html\#ta7 
APPENDIX A

WALLDESIGN PROGRAM 


\section{INTRODUCTION}

The WallDesign program carries out the transient heat transfer analysis of any composite structure along with optimization. The program was specifically made using Microsoft Visual Studio $\mathrm{C}++$ (2008) and is dependent on either ABAQUS v. 6.10 or the IBVP program. It cannot be run without the aid of one of those programs. The program only considers a 1D heat flow since all the problems are assumed to be one-dimensional problems.

\section{PROGRAM EXECUTION}

The input file name has to be specified as an essential command argument. Use the process below to enter the filename in the format mentioned. There are five options of the type of analysis that can be executed that include FE analysis from both ABAQUS or IBVP, post process only and optimization from both ANAQUS or IBVP. The FE analysis creates an ABAQUS model and then analyzes outputs the results of the given model. This is performed by using the "-abaqus" command in the command arguments. The post process only runs just the computational part of the energy analysis after the ABAQUS output file (*.dat) is already present. This is done by using the "-postprocess" command in the command arguments. The optimization of the model using ABAQUS can be performed using the "-optimizeA" command. The FE analysis is carried out using the IBVP program using the "-IBVP" command argument. The optimization using the IBVP program is performed by using the "-optimizel" command argument. 


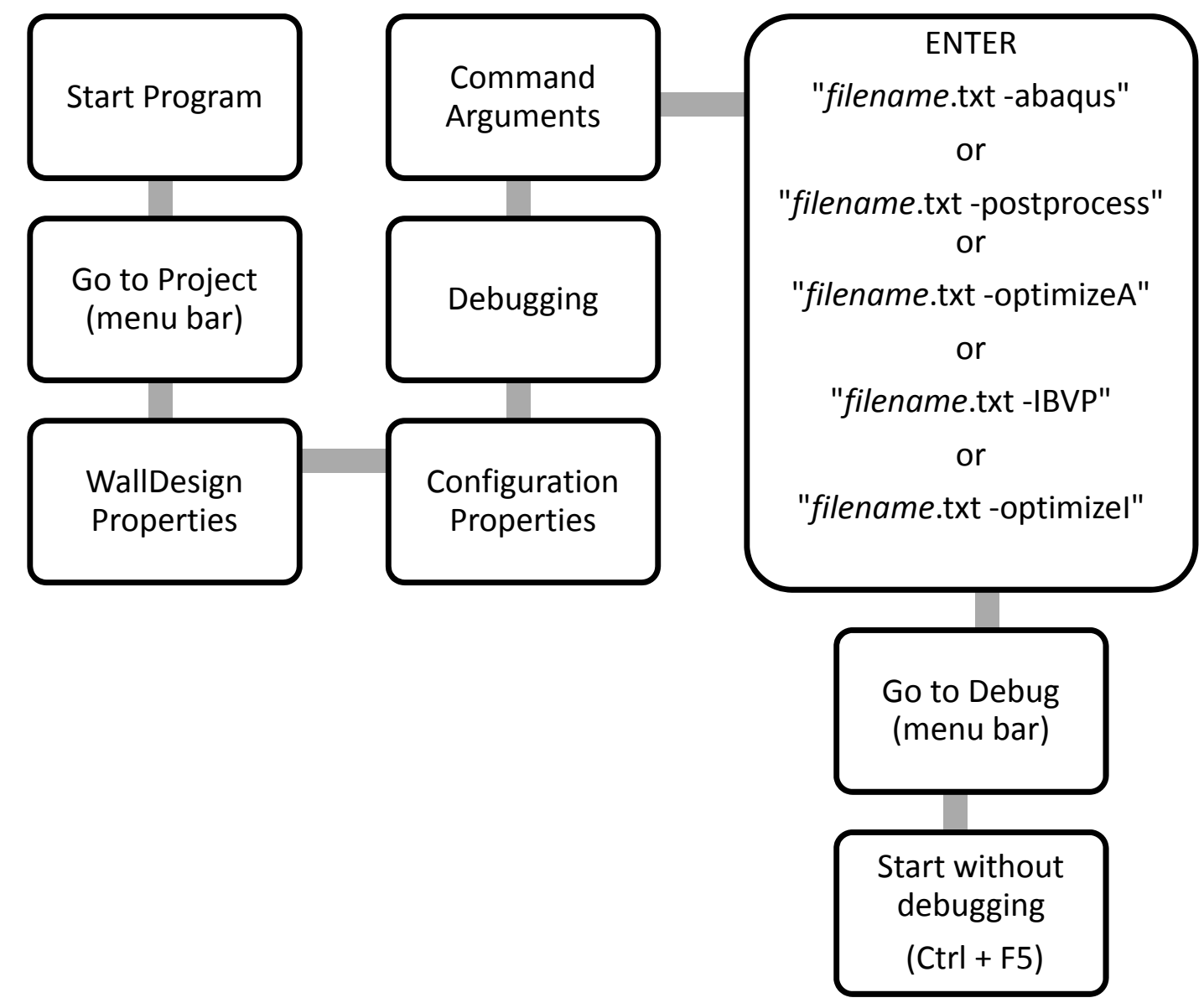

Figure A-1: Procedure to start program

\section{ALGORITHM FOR ABAQUS ANALYSIS}

1. Check number of input arguments and process accordingly

2. Check total number of runs and loop through each run separately

3. Open input and output files

4. $\quad$ Read data from Input ("*.txt") file

5. Write ABAQUS input file ("*.inp")

6. Launch ABAQUS and analyze the input file

7. ABAQUS creates the output file ("*.dat") from the heat analysis 
8. $\quad$ Read data from ABAQUS output file ("*.dat")

9. Start POST PROCESSING ABAQUS results

10. Loop through all time steps

11. Calculate the value of Energy flow through inside face (EFTIF) and Energy flow through outside face (EFTOF)

12. Loop though all elements

13. Calculate values for Latent energy, Sensible energy and Total energy stored in each element and sum them up for each time step

14. Print all values of energy to the output file

15. Optimize if appropriate (see the optimization section later)

16. Close all files and terminate program

\section{ALGORITHM FOR IBVP ANALYSIS}

1. Check number of input arguments and process accordingly

2. Check total number of runs and loop through each run separately

3. Open $\mathrm{I} / \mathrm{P}$ and $\mathrm{O} / \mathrm{P}$ files

4. Read data from Input ("*.txt") file

5. Set up data for IBVP program

6. Run IBVP program and get response values from IBVP

7. Start POST PROCESSING IBVP response results

8. Loop through all time steps

9. Calculate the value of Energy flow through inside face (EFTIF) and Energy flow through outside face (EFTOF) 
10. Loop though all elements

11. Calculate values for Latent energy, Sensible energy and Total energy stored in each element and sum them up for each time step

12. Print all values of energy to the output file

13. Optimize if appropriate (see the optimization section later)

14. Close all files and terminate program

\section{PROGRAM ORGANIZATION}

OutputBanner :

Prints a banner with license, time and date of analysis on each output file.

ABAQUSFEAOnly :

Runs all appropriate functions for carrying out the FE analysis in ABAQUS. Re-runs entire analysis for number of specified run times.

IBVPFEA :

Runs all appropriate functions for carrying out the FE analysis in IBVP. Re-runs entire analysis for number of specified run times.

PostProcessOnly :

Runs all appropriate functions for performing the post process analysis only from ABAQUS output file. Re-runs entire analysis for number of specified run times.

\section{OptimizeABAQUS :}

Runs all appropriate functions for carrying out the optimization process using

$$
\text { ABAQUS as the FEA }
$$

OptimizeIBVP: 
Runs all appropriate functions for carrying out the optimization process using IBVP as the FEA

\section{Interpolate :}

Function to interpolate between any given set of $x-y$ values, when called. VerifyCommandLineInput :

The function adds the non-optional input file name along with either "-abaqus", "postprocess", “-optimizeA", “-IBVP” or “-optimizel” arguments to the program. The "-abaqus" lets FE analysis in ABAQUS take place. The "-postprocess" option lets you skip the ABAQUS analysis and carry out only post-processesing. The " optimizeA" lets optimization of the model take place using ABAQUS. The "-IBVP" lets FE analysis in IBVP take place. The "-optimizel" lets optimization of the model take place using IBVP. This Function also opens the input file.

\section{OpenOutputFiles :}

Opens the output files based on the debug level.

\section{TerminateProgram and CloseAllFiles :}

Closes all files and exits the program.

\section{ReadInputFile :}

Reads the input ( "*.txt" ) file and stores all data required. The function runs through the input file twice, the first time to set all the variable sizes and get a rough estimate of the number of parameters. During the second pass all the data is stored in the required structure.

Analyze :

*Only used if performing ABAQUS FEA. 
According to the size of the elements given or the number of elements through thickness given in the input file, a mesh is generated (i.e. nodal and elemental data for the FE analysis in ABAQUS are generated) and the data is printed to the ABAQUS input file ("*.inp"). Also, the input file is completed with all time steps, material data and boundary conditions. After completion of the input file the ABAQUS command program is launched and the input file is processed to create the FE model and ABAQUS output file to use for postprocessing.

\section{PostProcess :}

After ABAQUS analysis is complete, the function reads the nodal temperature and elemental heat flux data from the ABAQUS output file ("*.out") and stores it in an appropriate structure.

ComputeStoredEnergy_Version2:

This function carries out all the post-processing procedures mentioned in the next section for ABAQUS FEA.

IBVPSetUp:

Sets up the data structure to carry out the FEA in the IBVP program. IBVPAnalyze:

Uses the IBVP program to solve the problem and compute the response values. ComputeEnergyIBVP:

This function carries out all the post-processing procedures mentioned in the next section for the IBVP FEA.

ErrorHandler : 
The error handler function detects any errors in the input file, analysis or the postprocesses and exits the program with the appropriate error message.

\section{POST PROCESSING}

After the finite element analysis is complete, either in ABAQUS or IBVP, the data for the nodal temperatures and heat flux at every point for all time steps is known from the result of each FEA. The Wall Design program then stores and processes this data and creates another set of energy related outputs, in either of the Compute Energy functions. The program runs a nested loop for each time step and each element. Energy stored in the form of sensible heat, latent heat and the total energy stored are calculated separately for each element, and then summed over all elements for each time step. Output from the program gives cumulative sensible energy, cumulative latent energy and step-wise total energy stored in each layer during each time step. From the output data, the maximum value of the latent energy stored in PCM is used to compute efficiency. Energy flow through the inside and outside walls is calculated using the heat flux data from the ABAQUS output file. For each set of either outer or inner elements, the flux obtained is multiplied by the (outer or inner) surface area to obtain energy flow through each surface for every time step. If the flux is negative (i.e. heat flows into the wall on the inside and out of the wall on the outside) energy flow is also considered to be negative. The positive and negative energy flow through the (outer and inner) face of the wall is tabulated separately in the output file for every time step, summation of which gives us the total energy flow [35]. 


\section{OPTIMIZATION}

The program is executed the same as previously stated, the "-optimizeA" or " optimizel" command will need to be used in the command line. This allows the program to run through the optimization process until the convergence criteria has been met.

\section{ALGORITHM FOR OPTIMIZATION}

1.Take the given design variables from the input file and manipulate

2.Run ABAQUS or IBVP and determine the new values for the heat flow through the model

3.Calculate the objective function

4.Manipulate the variables further and repeat Step 2

5.Once the convergence criteria is met the best feasible design is output as the result

\section{OPTIMIZATION ORGANIZATION}

FEWrapper :

Evaluates wrapper function.

\section{GEWrapper :}

Evaluates gradient function (not needed for GA or DE).

Wall_Design:

Determines how many evaluations have been called and if convergence has taken place.

Wall_DesignINIT: 
Sets the initial values for the design variables along with the bounds and precision values.

Wall_DesignFE:

Performs the new FE Analysis using the new design variables and calculates the fitness function and computes the constraint values to determine if the design is converging and feasible

UpdateValues :

Updates the design variables to use in the next FEA.

\section{PRODUCING BETTER OPTIMIZATION RESULTS}

To better optimize a problem several things can be done:

1.Ensure the objective function is stated correctly (should be to minimize)

2.Ensure the proper constraints are being used and try tightening the constraints slightly (all constraints should be less than and equal to zero)

3.Tighten the bounds given on the initial design variables

4.Try a larger amount of iterations

5.Try a larger population size

\section{OUTPUT FILES}

The program WallDesign creates 5 different output files. If the Debug Level of the program is set to 0 , only the first two output files are generated. If the debug level is set to 1 , all the output files are generated [35].

1) filename_Output.out 
This is a text file which stores Energy flow through outer and inner face and the energy stored by each layer for every time step. It also outputs the energy storage capacity of the PCM in a single line at the bottom.

2) filename_OutputALL.xls

This is an excel (2003-2007) format output file that stores the below data only for the output increments desired.

Time Step, Sensible heat stored, Latent heat stored, Energy flow through Inner face (EFTIF),PCM Efficiency (PCME), Outer face temp, interface temp's for all layers, Inside face temp., Temp. of nodes just before the inside face, Left end applied temp., Right end applied temp.

3) filename_NT.xls

This is an excel (2003-2007) format output file that stores the Nodal temperature data for the outer, inner and interface nodes for all time steps.

4) filename_OutputEnergy.xls

This is an excel (2003-2007) format output file which stores the cumulative values of sensible energy, latent energy, total energy stored in the PCM respectively.

5) filename_Output1.xls

This is an excel(2003-2007) format output file that stores the following data, for each element for each time step. Values output are only for the particular element. Time Step, Layer No., Element \#, Initial Temp, final Temp, Delta T, Sensible heat stored, Latent heat stored, Total energy stored (cumulative), specific heat capacity, Latent heat per degree, mass of element. 


\section{INPUT FILE FORMAT}

*heading

Analysis of concrete wall

*units

length, $m$

mass, $\mathrm{kg}$

time, $s$

temperature, $K$

energy, $J$

* parameter

**name, value

H, 1.0

$T$ CONCRETE, 5

(Height of model)

$T P C M, 5$

(thickness of Layer 1)

E_CONCRETE, 2

(thickness of Layer 2 and so on)

(\# of elements through thickness

desired for Layer 1)

E_PCM, 2

(\# of elements through thickness

desired for Layer 2 and so on)

*sel, value

Constant value of element thickness

(to be used instead of value of \# of

elements through thickness)

*debug level, 0 or 1

Value of 0 or 1 will decide which

output files are to be printed

*run, 1, Name1

\# of sets of different BC's to be applied

and name of file to be printed

*dimensions

**Height $\mathrm{x}$ Thickness

H, T_CONCRETE +T_PCM

Parameters used for denoting height and total thickness of model

*table, name1, \# of rows, \# of

Time to be mentioned only if $x$-data is columns, Time

time

$x 1, y 1$

$x 2, y 2$

$x 3, y 3$ and so on

*table, name2, \# of rows, \# of

columns, Time

$x 1, y 1$

$x 2, y 2$

$x 3, y 3$ and so on

*layer

Define layer name, geometry and material properties

**name, thickness, number of elements through thickness

Concrete, $T$ Concrete, $E$ Concrete

**density, conductivity or table name, specific heat or table name 
$2400.0,1.45,750.00$

**latent, solidus temp, liquidus temp (replace with 0 if no value is to be assigned),

$0,0,0$

Repeat layer data for all layers

*initial temperature

Initial condition of model

1 , value

Run \# and initial temperature

*time steps

**\# of time steps, final time value, output increment start, output increment end

$48,172800,25,48$

Final time value should be in consistent units. Output will be

displayed on for the increments

mentioned

*left end bc

**Run \#, type, table name, value 1 , value 2

* 1, mixed, Table1, 1.0, 20.0

If $B C$ type is mixed, and temperature varies over time. Value 1 is the base value for which amplitude is specified, Value 2 is heat transfer co-efficient for left end

*1, mixed, , 300.0, 20.0

If $B C$ type is mixed, and temperature remains constant over time. Value 1 is the temperature, Value 2 is heat transfer co-efficient for left end

$* 1, e b c,, 300.0$ If $B C$ type is EBC. Value 1 is the fixed temperature

* $1, n b c,, 1000.0$

If BC type is NBC. Value 1 is the value of heat flux (negative value indicates heat flow out of model), Value 2 not required

Repeat above line for all runs

*right end bc

** Run \#, type, table name, value 1 , value 2

* 1, mixed, Table1, 1.0, 20.0

(same rules apply as in Left end BC)

*1, mixed, , 300.0, 20.0

* $1, e b c,, 300.0$

* $1, n b c,, 1000.0$

* postprocess

Repeat above line for all runs

stored_energy, PCM

energy flow, rightend

* mat table, name1, \# of rows, \# of First material table columns 
**Material Name, Density, Conductivity, Specific Heat, Latent Heat, Solidus Temp, Liquidus Temp, Cost

Conc 1, 2400.0, 1.45, 750.0, 0, 0, 0, 503.10

**Note: The material properties of each layer described above must be

material 2, value 1, value 3, value a material found in one of the material $3 \ldots$ tables **

material 3, value 1, value 2, value 3 and so on

*mat table, name2, \# of rows, \# of columns

**Material Name, Density, Conductivity, Specific Heat, Latent Heat, Solidus Temp, Liquidus Temp, Cost

material 1, value 1, value 3, value $3 \ldots$

material 2, value 1, value 3, value $3 \ldots$

material 3, value 1 , value 2 , value 3

and so on

*iterations

$* *$ number of iterations

10

*method

**method of optimization

GA or $D E$

$G A$

*population size

25

*layer optimization

Define which layers are being optimized for thickness and/or

**layer number, material and the bounds

THICKNESS/NONE, MATERIAL

TABLE/NONE, PROPERTY/NONE, bounds

1, THICKNESS, mat table 1, NONE, $0.001,0.1,1,4$

Repeat
required

material and the bounds 
2, THICKNESS, NONE, NONE, If only thickness is a design variable $0.001,0.1$ then the $5^{\text {th }}$ and $6^{\text {th }}$ items are the bounds on thickness

3, NONE, mat table 2, DENSITY, If material is a design variable and it 750,850 is selected between a certain range of values for a property then the $6^{\text {th }}$ and $7^{\text {th }}$ items are the bounds on the property values to use from the given table

4. THICKNESS, mat table 1 , If thickness and material are design CONDUCTIVITY, $0.001,0.1,0.15$, variables and a property range is 0.19

important then the $5^{\text {th }}$ and $6^{\text {th }}$ values are the bounds for the thickness and the $7^{\text {th }}$ and $8^{\text {th }}$ values are the bounds on the property values to be used from the given table

5, NONE, mat table 2, NONE, 2, 3 If material is the only design variable and a property range is not important then the $5^{\text {th }}$ and $6^{\text {th }}$ values are the bounds on the materials to use from the given material table

**Note: the properties that can be used include: DENSITY, CONDUCTIVITY, SPECIFIC HEAT, LATENT HEAT or MELTING TEMP

Repeat for all layers to be optimized

*constraints Define which layers are being optimized for thickness and/or material and the bounds

**THICKNESS, \# of values, coefficient, layer \#, coefficient, layer \#...

THICKNESS, 3, 1.0, 1, 1.0, 2, 1.0, 3

The constraint is on the thickness and involve layer 1, 2 and 3 all with coefficients of 1.0

greater than, 0.20

*objective function

The constraint can be greater than or less than the allowable value Repeat both lines for all constraints Define the cost of each material used in the objective function and the time period for life cycle analysis

\footnotetext{
**material name/energy, cost/time concrete, cost energy, cost PCM, cost
} 
time, amount of time

*end

Indicates last line of input file

\section{Optimization Example:}

Input file has been written for a $20 \mathrm{~cm}$ thick concrete wall ( $1 \mathrm{~m}$ in height) with a $1 \mathrm{~cm}$ PCM layer placed at the center of the wall. Ambient air temperature acts on the outside face, i.e. mixed $\mathrm{BC}$ over 24 hours. Only one day with varying ambient temperatures on the outside and inside have been applied to the wall. Initial temperature of the wall for is 296 K. Outside and inside heat transfer coefficients are 20 and $5 \mathrm{~W} / \mathrm{m}^{2}-\mathrm{K}$ respectively. Output is desired only from the second 24 hours. Element size is fixed at $0.5 \mathrm{~cm}$. There will be 8 iterations performed on a population of 25 for a total of 200 fitness evaluations. The second layer will be optimized for thickness and material selection for the PCM material table. The third layer will be optimized for material selection from the concrete material table. The constraints are placed on the overall wall thickness to be between $20 \mathrm{~cm}$ and $25 \mathrm{~cm}$.

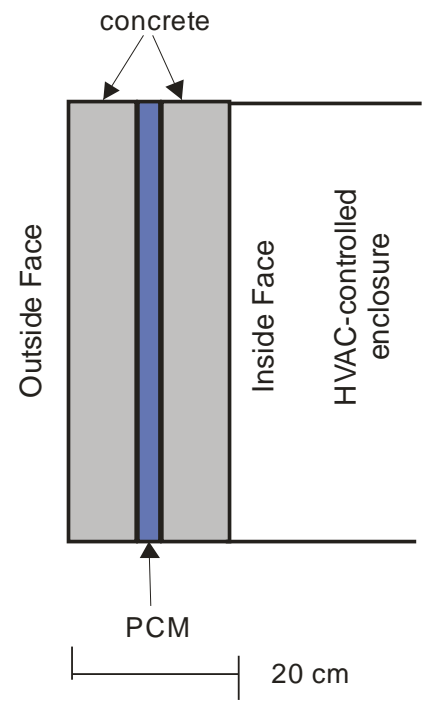

Figure A-2: Model details for sample input file 


\section{SAMPLE INPUT FILE:}

*heading Optimization of a $20 \mathrm{~cm}$ Wall - $5 \mathrm{~cm}$ PCM placed in center of wall

*units

length, $\mathrm{m}$

mass, $\mathrm{kg}$

time, $s$

temperature, $\mathrm{K}$

energy, $\mathrm{J}$

$* * * * * * * * * * * * * * * * * * * * * * * * * * * * * * * * * * * * * * * * * * * * * * * * * * * * * * * * * * *$

$* *$

* parameter

** name, value

$\mathrm{H}, 1.0$

T_CONCRETE1， 0.075

T_PCM, 0.05

T_CONCRETE2， 0.075

E_CONCRETE1， 2

E_PCM, 2

E_CONCRETE2, 2

* sel, 0.005

*debug level, $\odot$

* run, 1, -1

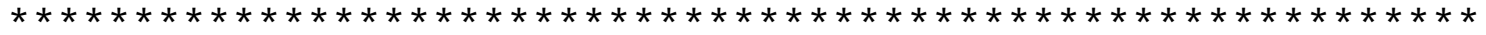

$* *$

*dimensions

**Height $x$ thickness

$\mathrm{H}$, T_CONCRETE1+T_PCM+T_CONCRETE2

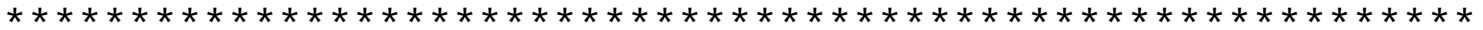

**

*table, conV1, 48, 2, TIME

3600

317.2

$7200, \quad 320.2$

$10800, \quad 321.2$

$14400, \quad 322.2$

$18000, \quad 323.2$

$21600, \quad 324.2$

$25200, \quad 324.2$

$28800, \quad 324.2$

$32400, \quad 324.2$

$36000, \quad 323.2$

$39600, \quad 322.2$

$43200, \quad 321.2$ 


\begin{tabular}{|c|c|c|}
\hline 46800 & 319.2 & \\
\hline 50400 & 318.2 & \\
\hline 54000 & 316.2 & \\
\hline 57600 & 314.2 & \\
\hline 61200 & 313.2 & \\
\hline 64800 & 311.2 & \\
\hline 68400 & 310.2 & \\
\hline 72000 & 310.2 & \\
\hline 75600 & 310.2 & \\
\hline 79200 & 311.2 & \\
\hline 82800 & 313.2 & \\
\hline 86400 & 315.2 & \\
\hline 90000 & 317.2 & \\
\hline 93600 & 320.2 & \\
\hline 97200 & 321.2 & \\
\hline 100800 & 322.2 & \\
\hline 104400 & 323.2 & \\
\hline 108000 & 324.2 & \\
\hline 111600 & 324.2 & \\
\hline 115200 & 324.2 & \\
\hline 118800 & 324.2 & \\
\hline 122400 & 323.2 & \\
\hline 126000 & 322.2 & \\
\hline 129600 & 321.2 & \\
\hline 133200 & 319.2 & \\
\hline 136800 & 318.2 & \\
\hline 140400 & 316.2 & \\
\hline 144000 & 314.2 & \\
\hline 147600 & 313.2 & \\
\hline 151200 & 311.2 & \\
\hline 154800 & 310.2 & \\
\hline 158400 & 310.2 & \\
\hline 162000 & 310.2 & \\
\hline 165600 & 311.2 & \\
\hline 169200 & 313.2 & \\
\hline 172800 &,$\quad 315.2$ & \\
\hline *table, & CONV2, 48, & 2, TIME \\
\hline 3600 & , 293 & \\
\hline 7200 & 293 & \\
\hline 10800 & 293 & \\
\hline 14400 & 293 & \\
\hline 18000 & 293 & \\
\hline 21600 & 293 & \\
\hline 25200 & 293 & \\
\hline 28800 & 293 & \\
\hline
\end{tabular}




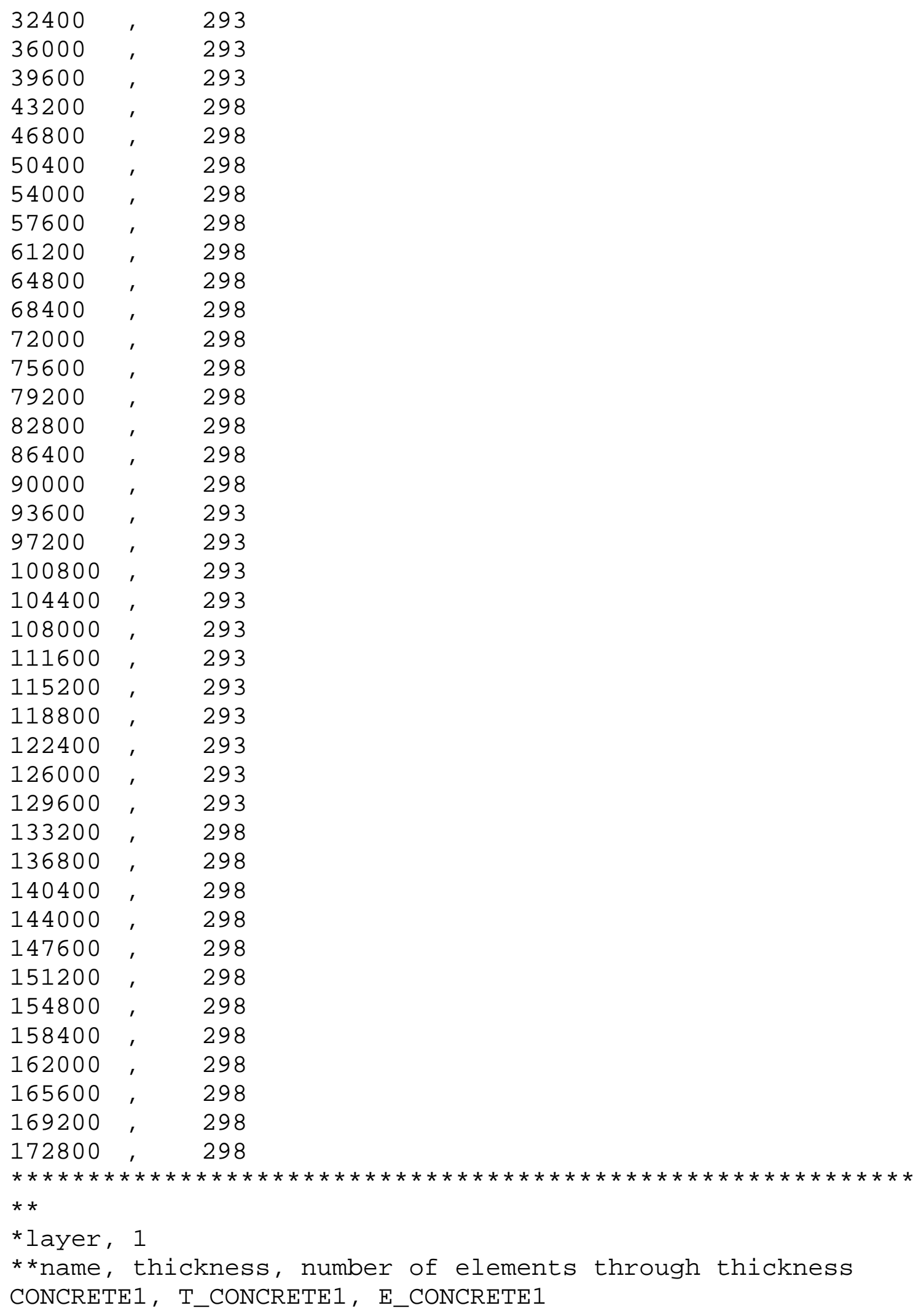


**density, conductivity or table name, specific heat or table name

$2400.0,1.45,750.0$

**latent heat, solidus temp, liquidus temp

$\odot, 0,0$

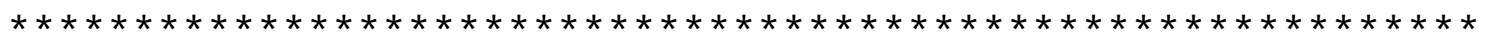

**

*layer, 2

**name, thickness, number of elements through thickness

PCM, T_PCM, E_PCM

**density, conductivity or table name, specific heat or table name

$800,0.2,2400$

**latent heat, solidus temp, liquidus temp $169000,300,304$

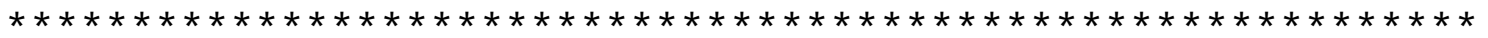

$* *$

*layer, 3

**name, thickness, number of elements through thickness

CONCRETE2, T_CONCRETE2, E_CONCRETE2

**density, conductivity or table name, specific heat or table name

$2400.0,1.45,750.0$

**latent heat, solidus temp, liquidus temp

$\odot, \Theta, 0$

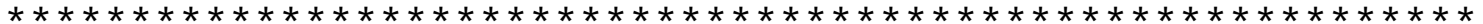

**

*initial temperature

$1,296.0$

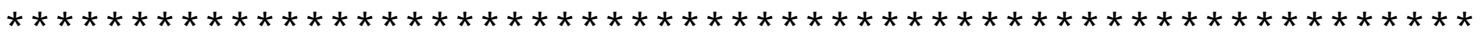

$* *$

*time steps

**\# of time steps, final time value, interested increment beg and end

$48,172800,25,48$

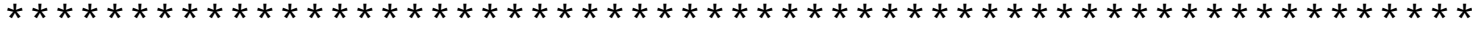

**

* left end bc

**type, table name, value 1 , value 2

1 , mixed, CoNV1, 1.0, 20.0

$* * * * * * * * * * * * * * * * * * * * * * * * * * * * * * * * * * * * * * * * * * * * * * * * * * * * * * * * * * *$

$* *$

*right end bc

**type, table name, value 1, value 2

1, mixed, CoNV2, 1.0, 5.0 


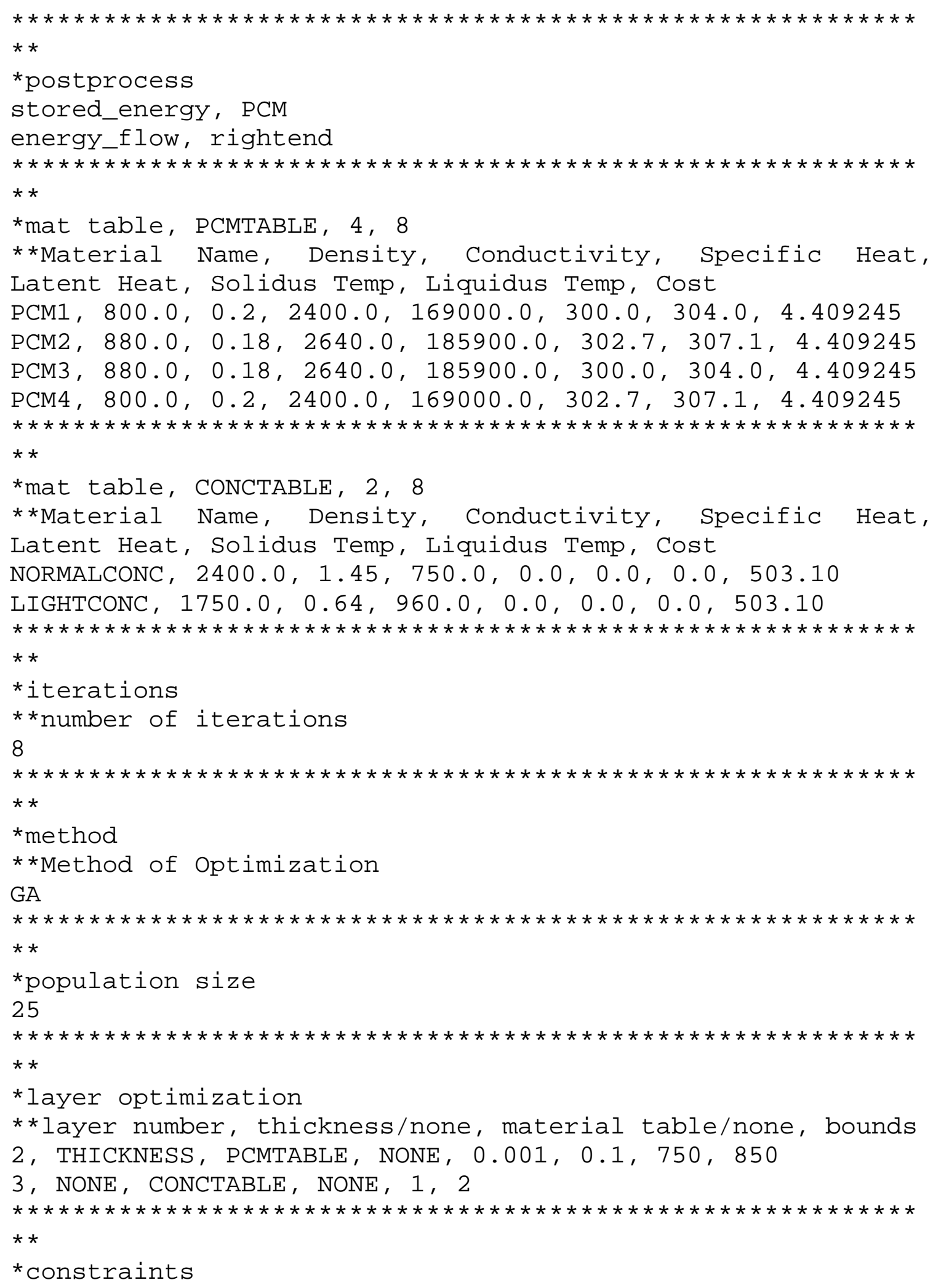


**THICKNESS, \# of values, coefficient, layer \#, coefficient, layer \#....

THICKNESS, 3, 1.0, 1, 1.0, 2, 1.0, 3

greater than, 0.20

THICKNESS, 3, 1.0, 1, 1.0, 2, 1.0, 3

less than, 0.25

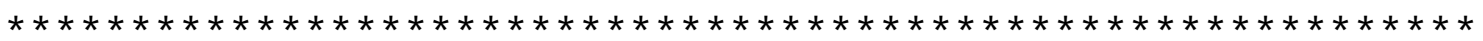

$\star *$

*objective function

**Material name/energy, cost/time

concrete, 503.10

PCM, 4.409245

energy, 0.1484

time, 3650

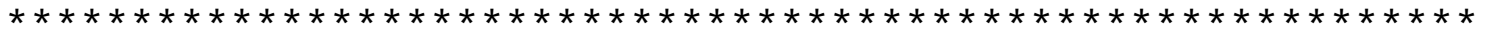

$* *$

*end 
APPENDIX B

IBVP PROGRAM 


\section{INTRODUCTION}

The IBVP program also functions as a stand along program to perform linear or nonlinear transient finite element analysis. The program was made using Microsoft Visual Studio $\mathrm{C}++$ (2008). This program also only considers a 1D heat flow.

\section{PROGRAM EXECUTION}

The input file name has to be specified as an essential command argument. Use the process below to enter the filename in the format mentioned. There is only one option of the type of analysis that can be executed and that is FEA. The use of "-fea" is used to perform the finite element analysis.

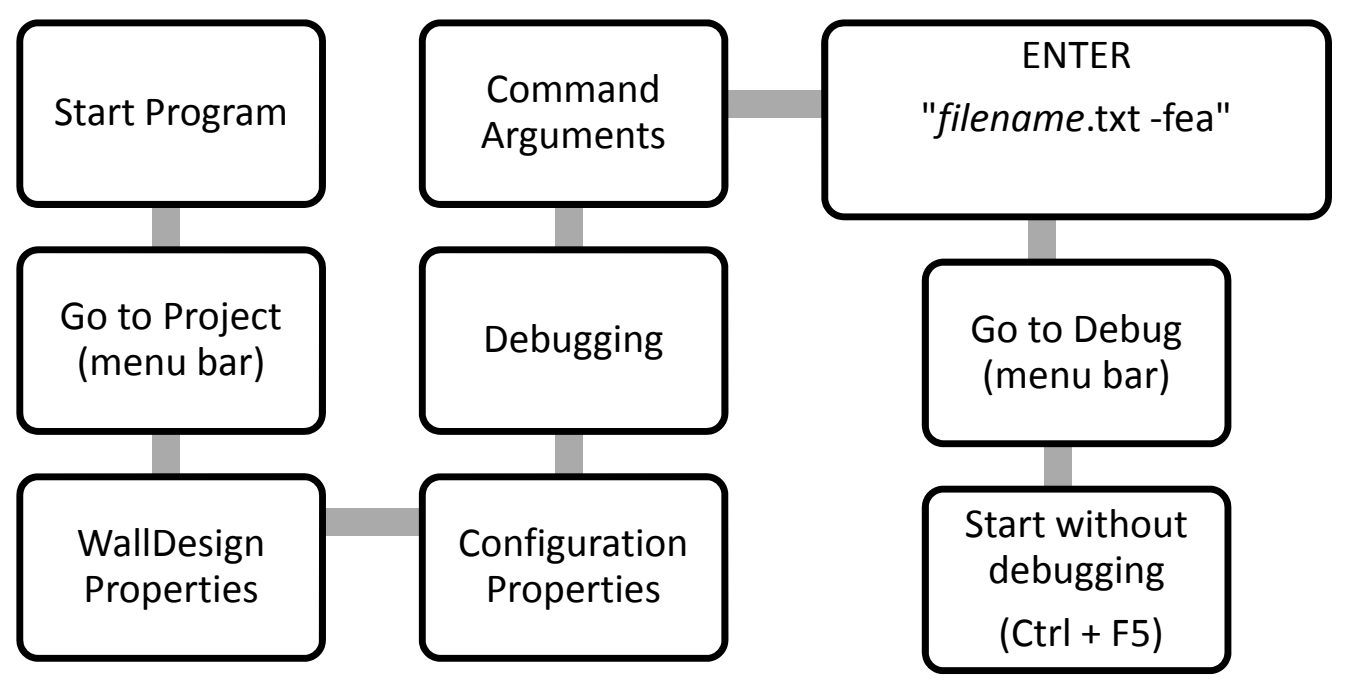

Figure B-1: Procedure to start program 


\section{ALGORITHM FOR FEA ANALYSIS}

1. Check number of input arguments and process accordingly

2. Check total number of runs and loop through each run separately

3. Open input and output files

4. $\quad$ Read data from Input ("*.txt") file

5. Set the size of all the data storage vectors and matrices needed

6. Loop through all time steps

7. Loop through all elements

8. Form the stiffness matrix

9. Form the load vector

10. Solve for the unknown temperatures

11. Compute element heat flux based on nodal temperatures

12. Print all temperatures and flux values to the output file

13. Close all files and terminate program

\section{PROGRAM ORGANIZATION}

\section{Initialize :}

Initializes all values to their standard values or 0 .

VerifyCommandLineInput :

Makes sure the input file name has been specified.

OpenOutputFiles :

Opens the output files.

CloseAllFiles : 
Closes all files.

ReadInputFile :

Reads the input ( "*.txt" ) file and stores all data required. The function runs through the input file twice, the first time to set all the variable sizes and get a rough estimate of the number of parameters. During the second pass all the data is stored in the required structure.

SetSize :

Sets the size of all the data and response matrices.

ImposeBC and ImposeBCNL:

Imposes the boundary conditions of the given problem for both linear and nonlinear problems.

SuppressDOF and SuppressDOFNL:

When an essential BC is used, the stiffness matrix is adjusted accordingly.

Solve:

Uses the stiffness matrix and load vector to perform Gaussian Elimination to solve for the nodal temperatures. Can be done for both linear and nonlinear problems using a Newton's loop for nonlinear problems.

ConstructK and ConstructKNL:

Creates the stiffness and capacity matrix based on shape functions for both linear and nonlinear problems.

ConstructF:

Creates the load vector.

ComputeResponse: 
Using the nodal temperatures found from Gaussian Elimination the heat flux values can be computed.

ErrorHandler:

If there is an error in the input file or the execution of the program it is exited with the appropriate error message.

EchoInpute:

Writes the input to the beginning of the input file.

CreateOutput:

Writes the results of the nodal temperatures and heat flux values to the output file.

\section{OUTPUT FILES}

The IBVP program creates 2 different output files.

1) filename.out

This is a text file that outputs the nodal temperatures and element flux for every node and every time step of the problem.

2) filename_XY.out

This is a text file that can be used with ASU Truss, a graphing program, to graph the results of the nodal temperatures. 


\title{
INPUT FILE FORMAT
}

\author{
*heading \\ Analysis of concrete wall \\ *debug level \\ 0 or 1 \\ *run, 1, Name1 \\ \# of sets of different BC's to be applied \\ and name of file to be printed \\ *segment, segment \#, thickness, \\ element type, \# of elements, property \\ \# \\ 1, 2, 1D-C0 linear, 10, 1 \\ 2, 1, 1D-C0 linear, 6, 2 \\ Repeat for as many segments as \\ required \\ *property, 1 \\ **density, conductivity or table name, specific heat or table name \\ $800,0.2,2400$ \\ **latent, solidus temp, liquidus temp \\ 169000, 312, 316 \\ replace with 0 if no value is to be \\ assigned) \\ *property, 2 \\ **density, conductivity or table name, specific heat or table name \\ $2400,1.45,750$ \\ **latent, solidus temp, liquidus temp \\ $0,0,0$ \\ *table, namel, \# of rows, \# of \\ Repeat property data for all properties \\ columns, Time \\ Time to be mentioned only if $x$-data is \\ $x 1, y 1$ \\ time \\ $x 2, y 2$ \\ $x 3, y 3$ and so on \\ *table, name2, \# of rows, \# of Repeat for as many tables as required \\ columns, Time \\ $x 1, y 1$ \\ $x 2, y 2$ \\ $x 3, y 3$ and so on \\ *initial temperature \\ 1 , value \\ Initial condition of model \\ *time steps \\ Run \# and initial temperature \\ **\# of time steps, time step increment, theta value \\ 20, 3600, 1.0 \\ Theta value must be between 0 and 1 \\ *left end bc \\ **Run \#, type, value 1 , value 2
}


* 1, Mixed, 20.0, name1

*1, Mixed, 300.0, 20.0

$* 1, E B C, 300.0$

* 1, NBC, 1000.0

* right end bc

** Run \#, type, value 1 , value 2

* 1, Mixed, 1.0, name2

*1, Mixed, 300.0, 20.0

* 1, EBC, 300.0

* $1, N B C, 1000.0$

* concentrated flux

** location, value

1, 10

*end
If $B C$ type is mixed, and temperature varies over time. Value 1 is heat transfer co-efficient for left end and Value 2 is the table name.

If $B C$ type is mixed, and temperature remains constant over time. Value 1 is heat transfer co-efficient for left end and Value 2 is the ambient temperature

If $B C$ type is $E B C$. Value 1 is the fixed temperature

If BC type is NBC. Value 1 is the value of heat flux (negative value indicates heat flow out of model), Value 2 not required

Repeat above line for all runs

(same rules apply as in Left end $B C$ )

Repeat above line for all runs

If no flux, then delete this line

Indicates last line of input file

\section{IBVP Example:}

The input file is written for a single layer of PCM that is $1 \mathrm{~m}$ thick with 10 elements across the thickness. There is a mixed boundary condition applied to left end as given by the table OUTSIDE and the right end is assumed to be insulated. The initial temperature of the layer is $310 \mathrm{~K}$. This analysis will be performed for 20 time steps with an step increment of $3600 \mathrm{~s}$ for a total time of 20 hours. The theta value that will be used is 1 . 


\section{SAMPLE INPUT FILE:}

*heading

One layer PCM

*debug level

1

${ }^{*}$ run, $1,-1$

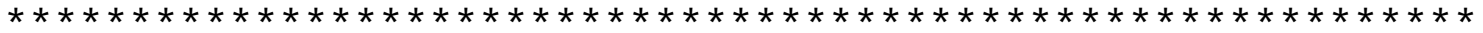

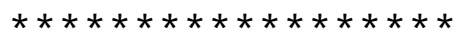

* segment

** segment\#, thickness, element type, \#ofelements, property\# $1,1,1 \mathrm{D}-\mathrm{CO}$ linear, 10, 1

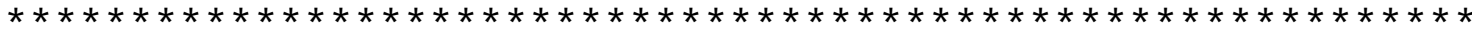

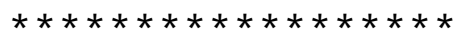

*property, 1

** density, conductivity or table name, specific heat or table name

$800,0.20,2400.0$

** latent heat, solidus temp, liquidus temp $169000,312,316$

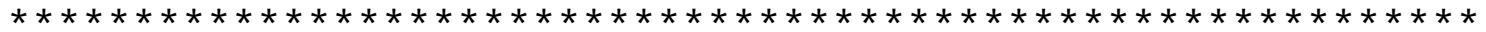

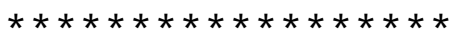

*table, OUTSIDE, 48, 2, TIME

$3600, \quad 317.2$

$7200, \quad 320.2$

$10800, \quad 321.2$

$14400, \quad 322.2$

$18000, \quad 323.2$

$21600, \quad 324.2$

$25200, \quad 324.2$

$28800, \quad 324.2$

$32400, \quad 324.2$

$36000, \quad 325$

$39600, \quad 325.4$

$43200, \quad 325.4$

$46800, \quad 326.3$

$50400, \quad 326.7$

$54000, \quad 327.2$

$57600, \quad 327.4$

$61200, \quad 327.4$

$64800, \quad 328$

$68400, \quad 328$

$72000, \quad 328.8$

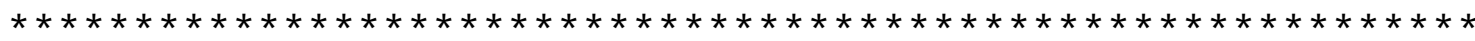

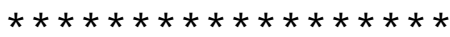


*initial temperature

**run \#, value

1,310

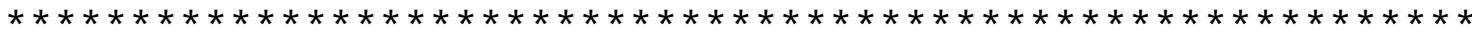

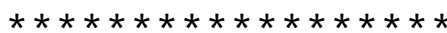

${ }^{*}$ timestep, 1

**Number of timesteps in interval, final time value, thetavalue

$20,3600,1.0$

${ }^{*}$ left end bc

** run \#, Type, value 1, value 2

1, Mixed, 20, OUTSIDE

*right end bc

** run \#, Type, value 1 , value 2

${ }^{*}$ concentrated flux

** location, value

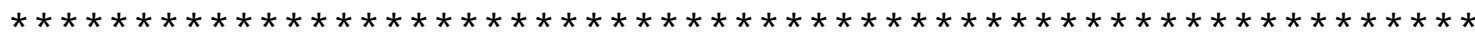

$\star \star * \star * * * * * * * * * * * * \star *$

*end 